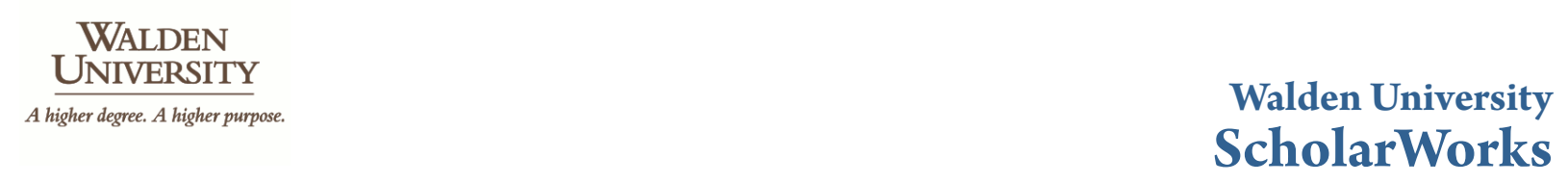

\title{
Predictors and Moderators of Sexual Distress in Women with Persistent Genital Arousal Disorder
}

\author{
Lisa Squibb \\ Walden University
}

Follow this and additional works at: https://scholarworks.waldenu.edu/dissertations

Part of the Medicine and Health Sciences Commons, and the Psychology Commons

\footnotetext{
This Dissertation is brought to you for free and open access by the Walden Dissertations and Doctoral Studies Collection at ScholarWorks. It has been accepted for inclusion in Walden Dissertations and Doctoral Studies by an authorized administrator of ScholarWorks. For more information, please contact ScholarWorks@waldenu.edu.
} 


\title{
Walden University
}

\author{
College of Social and Behavioral Sciences
}

This is to certify that the doctoral dissertation by

Lisa Squibb

has been found to be complete and satisfactory in all respects, and that any and all revisions required by the review committee have been made.

Review Committee

Dr. Brian Zamboni, Committee Chairperson, Psychology Faculty Dr. Lara Stepleman, Committee Member, Psychology Faculty Dr. Peggy Gallaher, University Reviewer, Psychology Faculty

Chief Academic Officer

Eric Riedel, Ph.D.

Walden University

2017 


\begin{abstract}
Predictors and Moderators of Sexual Distress in Women with Persistent Genital Arousal

Disorder

by

Lisa Squibb
\end{abstract}

MPH, Institute for the Advanced Study of Human Sexuality, 2012

MBA, Fairfield University, 2001

BS, Fairfield University, 1999

\author{
Dissertation Submitted in Partial Fulfillment \\ of the Requirements for the Degree of \\ Doctor of Philosophy \\ Health Psychology
}

Walden University

May 2017 


\begin{abstract}
Persistent Genital Arousal Disorder (PGAD) is a complex, poorly understood condition characterized by unremitting, unwanted, distressing genital arousal which occurs frequently and for extended periods of time, often in the absence of sexual stimuli. The pathophysiology is unknown. Researchers have hypothesized underlying disorders of neurological, vascular, pharmacological and psychological origins. Possible causalities have been suggested including anxiety disorder, SSRI use, and pudendal nerve neuropathies among others. Despite the uncertainty of etiology, other aspects of the disorder are clearer, including distress and poor quality of life for many. In this study, I used a biopsychosocial model to examine three potential psychological predictorsdepression, stress, and anxiety_as well as moderators including erotophobia and perceived partner support on a sample of 51 women with PGAD. Quantitative survey methodology was used to collect information from women with PGAD who had sought care for their condition from a sexual medicine specialty clinic (San Diego Sexual Medicine) and members of an online PGAD support group. Results of the study indicated depression as the most significant predictor of distress of the three psychological variables under study. Neither erotophobia nor perceived social support moderated any of the psychological factors studied. Scores on the Female Sexual Distress Scale—-Revised demonstrated clinically important levels of distress. The findings highlight the need for a biopsychosocial treatment approach to the condition, including psychological therapy. Implications for social change include the preservation of quality of life and reduced depression rates in women with PGAD.
\end{abstract}


Predictors and Moderators of Sexual Distress in Women with Persistent Genital Arousal Disorder by Lisa Squibb

MPH, Institute for the Advanced Study of Human Sexuality, 2012

MBA, Fairfield University, 2001

BS, Fairfield University, 1999

\author{
Dissertation Submitted in Partial Fulfillment \\ of the Requirements for the Degree of \\ Doctor of Philosophy \\ Health Psychology
}

Walden University

May 2017 


\section{Dedication}

I would like to dedicate this dissertation to my two sons, Aaron and Ryan, who have spent much of their childhood watching their mom strive to achieve this incredible goal. May they always have the faith, the drive and the strength to believe in themselves and reach for their goals, no matter how big or how small. I would also like to dedicate this to my husband, Dr. Michael Sand, who has given me an immense amount of support throughout this process, and to whom I owe an incredible debt of gratitude and thanks. His patience and guidance have held me up, given me strength and kept me going strong, even on my worst days. 


\section{Acknowledgments}

I would like to acknowledge my chair, Dr. Brian Zamboni, for all his guidance, leadership, patience, support, and feedback throughout my dissertation. Without his encouragement and words of wisdom, I could not have accomplished this incredible feat. Dr. Zamboni, you truly are an amazing mentor that I will forever hold dear in my thoughts and in my future work.

I would also like to extend my thanks to my committee member, Dr. Lara Stepleman, who has helped me formulate my ideas and put them into a body of work that I hope will have significant meaning for those both in the field of female sexual health and the women in need of more research in this and other areas of sexual health.

I would like to acknowledge Dr. Irwin Goldstein and his wife, Sue Goldstein, for assisting me with this process, and a heartfelt thank you for their willingness to go above and beyond in assisting with participant recruitment through their sexual medicine facility. I could not have done this dissertation without you both. I am forever in your debt.

Finally, I would like to thank the administrators of the PGAD online support group for allowing me to post my survey within their forum. I cannot thank them and the participants enough for their participation in PGAD research and for contributing to the literature that is so badly needed in this area. 


\section{Table of Contents}

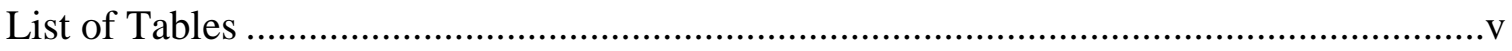

Chapter 1: Introduction to the Study ....................................................................

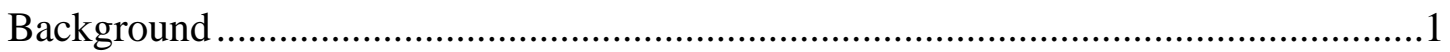

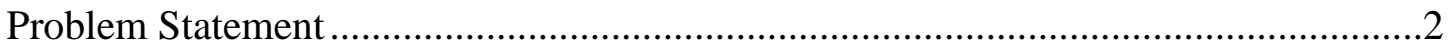

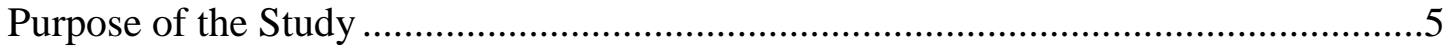

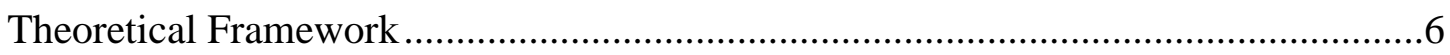

Research Questions and Hypotheses .................................................................

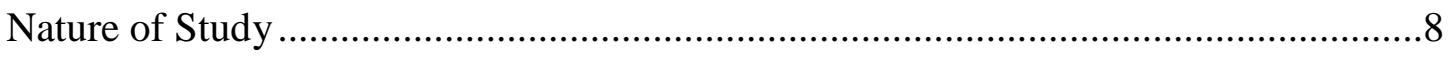

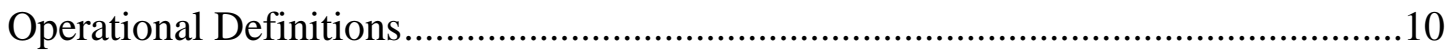

Assumptions, Limitations, and Delimitations......................................................11

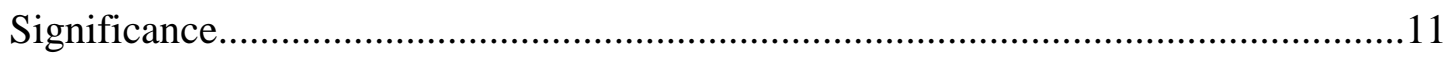

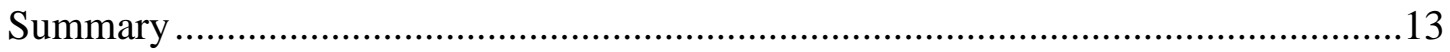

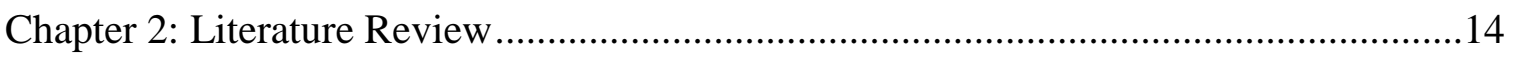

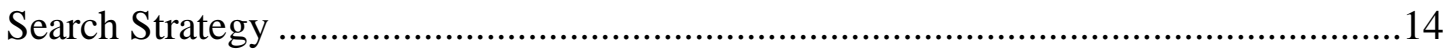

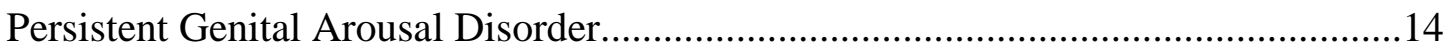

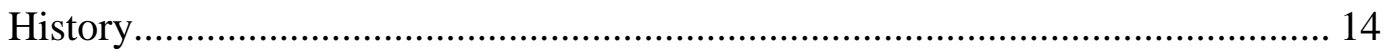

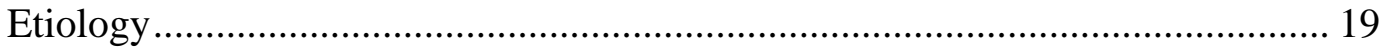

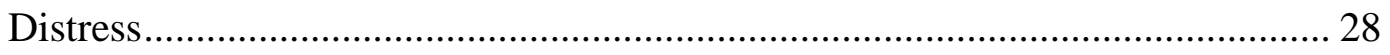

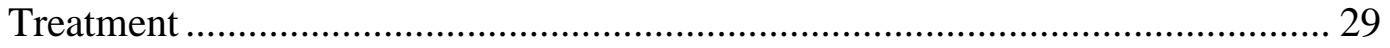

Depression, Anxiety and Stress in Sexual Dysfunction ..........................................32

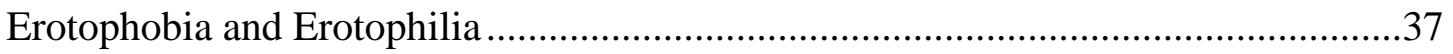




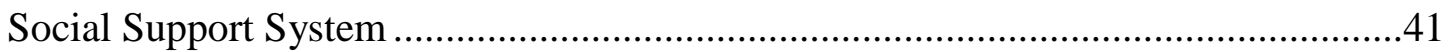

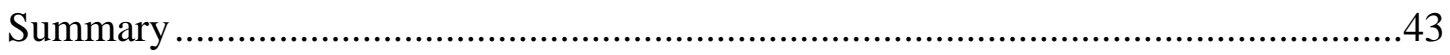

Chapter 3: Research Method.........................................................................................46

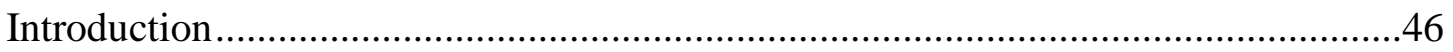

Research Design and Rationale ..........................................................................46

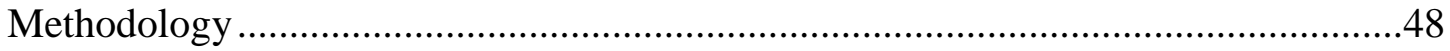

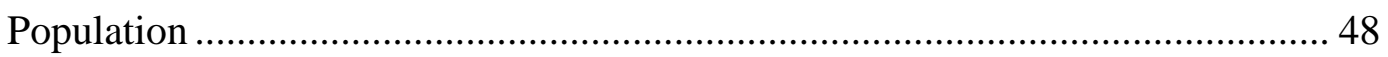

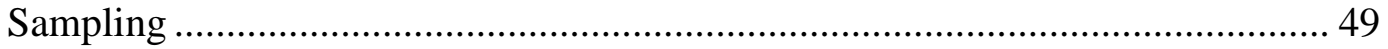

Data Collection Process ................................................................................ 50

Instrumentation and Operational Constructs .............................................................52

Dependent Variable -Distress........................................................................... 52

Independent Variables - Depression, Anxiety, and Stress .................................... 52

Moderating Variables - Erotophobia/Erotophilia and Perceived Partner

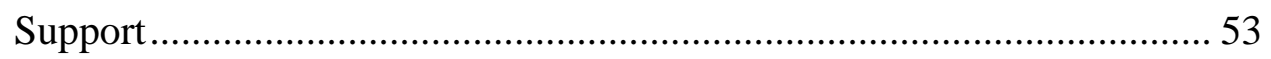

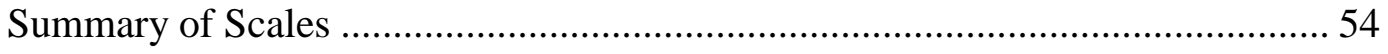

Demographics and Gynecological Information .................................................. 54

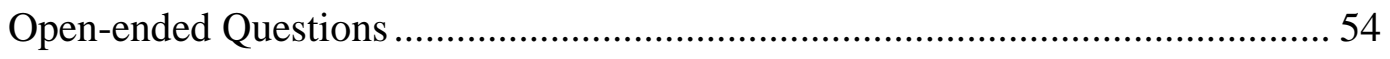

Research Questions, Hypotheses, and Statistical Analysis Methodology .....................55

Data Screening and Cleaning............................................................................. 55

Research Questions and Hypothesis ................................................................. 56

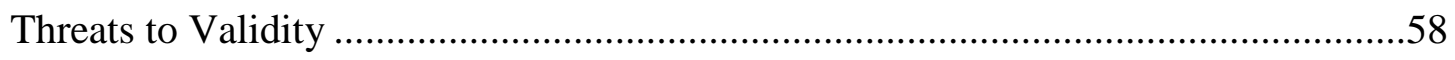

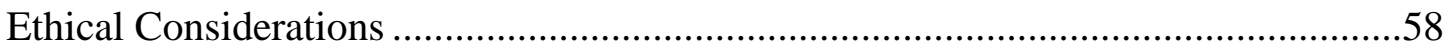


Summary

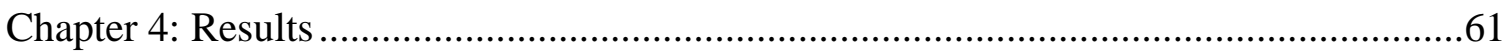

Introduction.................................................................................................61

Participant Demographic and Medical Information ..................................................61

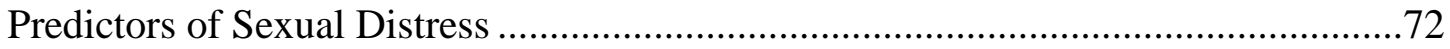

Hypotheses - Research Question 1................................................................... 72

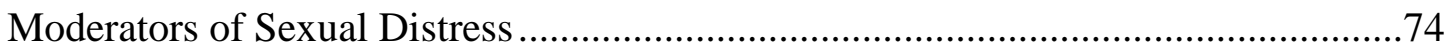

Hypotheses - Research Questions 2 and 3 ...................................................... 74

Women with Partners vs. Women without Partners .....................................................77

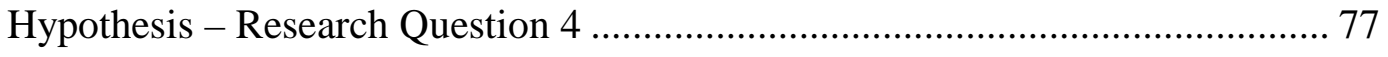

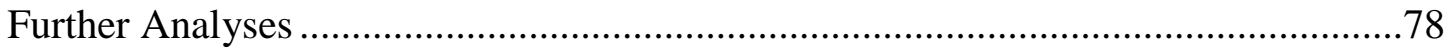

Comments from Open-Ended Questions ...................................................................79

First Open-Ended Question.................................................................................. 79

Second open-ended question.......................................................................... 80

Third open-ended question .......................................................................... 81

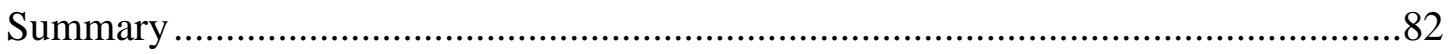

Chapter 5: Discussion, Conclusions, and Recommendations .............................................84

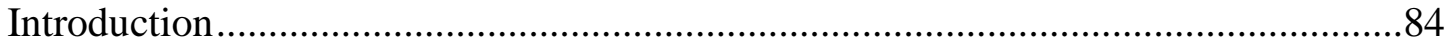

The Significant Predictors........................................................................................

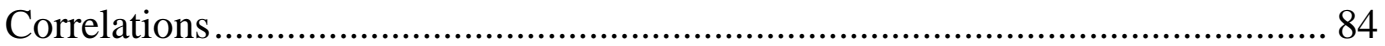

Depression Versus Anxiety and Stress as Predictors.............................................. 87

Theories Behind the Results ............................................................................ 88 
Understanding the Current Findings ................................................................. 89

Signals in the Medical Backgrounds and Comments ............................................ 92

Possible Connection to SSRIs/SNRIs .................................................................. 94

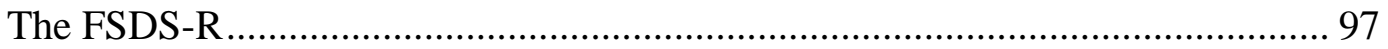

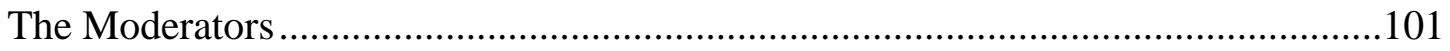

Erotophobia-Erotophilia .................................................................................. 101

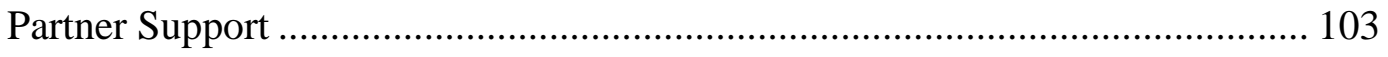

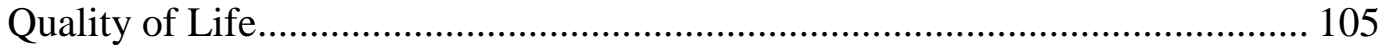

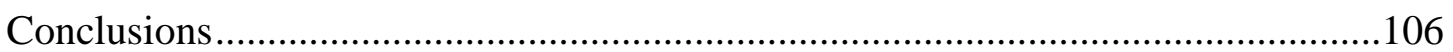

Limitations of the Study.....................................................................................107

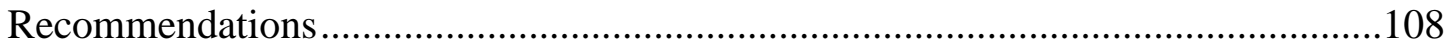

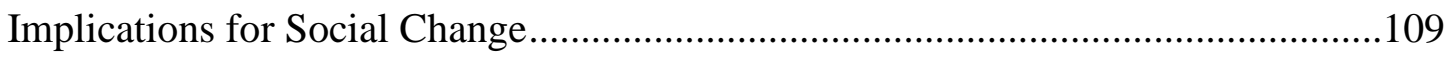

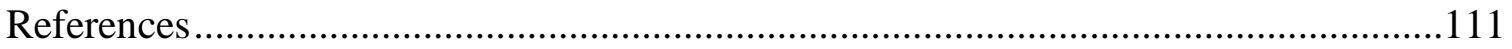

Appendix A: Scales and Questionnaires..................................................................132

Appendix B: Email Invitation to Participate...............................................................150

Appendix C: Online Support Group Invitation to Participate ............................................151

Appendix D: Seven-day Follow-up Email Invitation to Participate...................................152

Appendix E: Fourteen-Day Follow-Up Email Invitation to Participate ............................153

Appendix F: Seven-Day Follow-Up Online Support Group Invitation to

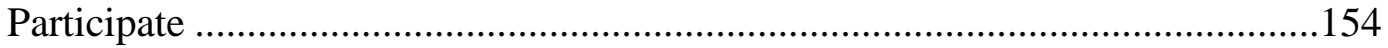

Appendix G: Fourteen-Day Follow-Up Online Support Group Invitation to

Participate 


\section{List of Tables}

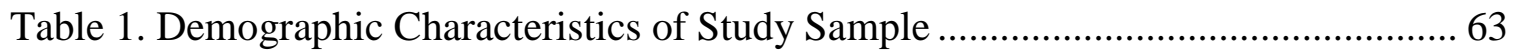

Table 2. Correlation Between Study Variables and Distress ....................................... 66

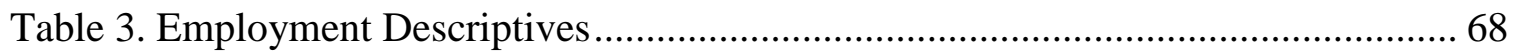

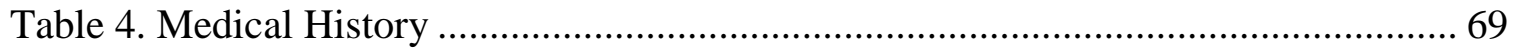

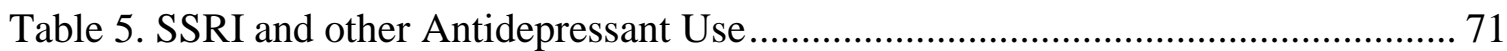

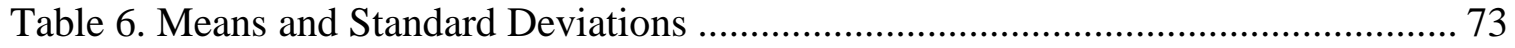

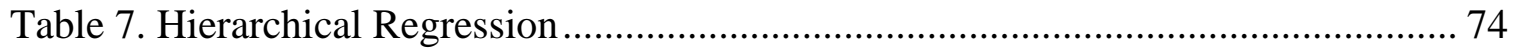

Table 8. Moderation Analysis of Erotophobia-Erotophilia ......................................... 76

Table 9. Moderation Analysis of Partner Support ........................................................ 77

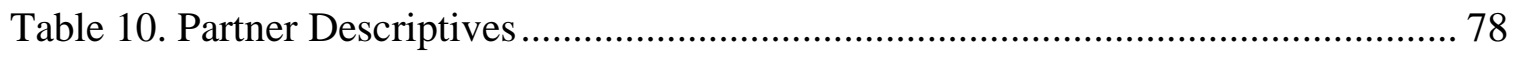

Table 11. Sexual Distress in SSRI and Other Antidepressant Use Comparison to Non-use 
Chapter 1: Introduction to the Study

\section{Background}

At the turn of the last century, a small group of women throughout the country were presenting to clinicians with symptoms previously undocumented in literature (Leiblum \& Nathan, 2001). These symptoms entailed feelings of persistent arousal in the genital area not accompanied by sexual desire or thoughts, and were deemed bothersome or distressful by the sufferers (Leiblum, Brown, Wan, \& Rawlinson; 2005). The women also indicated that the symptoms were often not relieved by one or more orgasms. Pain, including pelvic, genital, and clitoral has been noted in some PGAD patients (Pink, Rancourt \& Gordon, 2014). Leiblum and Nathan (2001) originally deemed the cluster of symptoms persistent sexual arousal syndrome, but after further research, renamed the cluster of symptoms as persistent genital arousal disorder (PGAD) because the arousal was contained in the genital area and was unrelated to sexual desire or thoughts.

Interestingly, significant variability in the distress women felt from PGAD symptoms was noted. Some women reported little or no distress at all, while others indicated severe distress to the point of being unable to function well socially or in everyday life tasks and situations (Leiblum \& Nathan, 2001). Reports have indicated that symptoms may be triggered and/or exacerbated by levels of anxiety, stress and/or depression (Leiblum \& Chivers, 2007; Leiblum, Seehuus, Golmeier \& Brown, 2007; Leiblum et al., 2005). The literature shows that women with moderate to severe distress are appearing in clinicians offices seeking treatment, making it important for researchers to focus on distress variability, including its correlates or predictors, its causes, and its consequences. 
Carvalho, Verissimo and Nobre (2015) recently completed a study examining contributors to distress including personality factors such as low openness to new experiences and ideas, neuroticism, and sexual beliefs. They noted that neuroticism, poor dyadic adjustment, sexually conservative beliefs, and low openness were significant predictors of distress; neither moderated the severity of PGAD symptoms and distress. Of these, sexually conservative beliefs were the most significant predictors of distress. Researchers have struggled to identify underlying causes of PGAD, and to date have yet to pinpoint a specific etiology. They have speculated myriad potential etiologies including neurological, pharmacological, psychological, and vascular pathologies (Bedell, Goldstein, \& Burrows, 2014; Waldinger, Venema, van Gils, \& Schweitzer, 2009; Leiblum \& Goldmeier, 2008; Goldmeier \& Leiblum, 2008). While prevalence is currently unknown, it is speculated that it may not be as rare as initially thought. More women may experience similar symptoms and distress, but abstain from reporting to their clinicians out of embarrassment, shame, guilt, or cultural taboo (Leiblum, 2008; Mahoney \& Zarate, 2007). The prevalence may also be low because women presenting with symptoms may be inadvertently diagnosed with other conditions instead of PGAD.

\section{Problem Statement}

PGAD, a condition characterized by unwanted, bothersome genital arousal causing distress in the absence of sexual desire or stimuli and often not relieved by orgasm, was first identified as a clinical syndrome by Leiblum and Nathan (2001). Five criteria make up the components of PGAD including:

1. Persistent feelings of arousal extending over days, weeks, or months at a time. 
2. The arousal is not alleviated by or only temporarily relieved by orgasm, sometimes requiring multiple orgasms.

3. The arousal is not associated with sexual thoughts or desire.

4. The arousal may be triggered by nonsexual stimuli or no apparent stimuli.

5. The arousal is bothersome and causes distress.

Currently, only one case report of isolated PGAD has been presented in the literature regarding a male who presented with "persistent feelings of impending orgasm" similar to arousal and without priapism or sexual desire; and two cases of restless genital syndrome have been reported in males, but not as isolated PGAD (Stevenson \& Kohler, 2015; Waldinger, Venema, van Gils, de Lint, \& Schweitzer, 2011).

Distress, as noted above, is a key component of the five diagnostic criteria of PGAD. It is not completely clear why some women experience high degrees of distress while others experience little or none at all. In the limited research available, researchers have hypothesized that negative appraisal, high cognitive awareness, and sensitivity to one's physical sensations causing greater anxiety are related to the differences in distress (Leiblum \& Chivers, 2007; Leiblum, Seehuus, Goldmeier, \& Brown, 2007). Researchers have also suggested that feelings of shame, guilt, embarrassment and worry contribute to higher levels of distress (Leiblum et al., 2005). The aforementioned study conducted most recently by Carvalho, Verissimo and Nobre (2015) targeted neuroticism, poor dyadic adjustment, low openness, and sexually conservative beliefs as potential distress triggers for some women. Because most of the research on female sexual arousal and dysfunction has focused on deficiencies in arousal, the phenomenon of persistent, 
distressful arousal continues to be a mystery. Considering the hypotheses and findings in the work by Leiblum and Chivers (2007), Leiblum, Seehuus, Goldmeier and Brown (2007), and Carvalho, Verissimo and Nobre (2015), it is reasonable to theorize and to promote further research regarding a connection between PGAD distress levels and psychological factors such as anxiety, depression and stress, and sexually conservative belief systems whereby negative/conservative (erotophobic) beliefs and positive/liberal (erotophilic) beliefs may play a role.

Distress, with regards to sexual dysfunction, has been a matter of discussion for many of the top researchers in the field (Hendrickx, Gijs, \& Enzlin, 2013). There are a host of arguments for keeping distress as a criterion for sexual dysfunction disorders, and just as many for taking it out. Some researchers argue that distress is a consequence of a sexual dysfunction and is based on clinician or expert opinion as opposed to empirical data, and that measuring it tends to complicate research. Others argue that, per empirical research, distress is not a factor or consequence for all women or men who experience sexual difficulty or dysfunction, and that it should be used to identity and work with individuals who desire treatment and seek out treatment options (Hendrickx, Gijs, \& Enzlin, 2013). With so much variability in levels of distress, some researchers have taken to assessing stress separately from sexual difficulties, arguing that a person can have a sexual dysfunction without having associated distress (Hendrickx, Gijs, \& Enzlin, 2013). Leiblum and Chivers (2007) identified this concept in their study of two populations: women with persistent genital arousal disorder who were distressed, and women with persistent genital arousal who were not distressed. 
Researchers have proposed several theories to explain the etiology of PGAD, such as the use of certain centrally-acting drugs such as SSRIs/SNRIs (Leiblum \& Goldmeier, 2008), dysfunction of the peripheral neuroanatomy (Bedell et al., 2014; Waldinger et al., 2009), vascular concerns (Graziottin, 2004; Thorne \& Stuckey, 2007), and psychological factors such as a subtype of anxiety disorder (Goldmier \& Leiblum, 2008). To date, all

of these theories have limited empirical support, precluding definitive statements regarding the etiology of the condition. Further, research to date has been hampered by the relative rarity and social stigmatization of the condition, as well as by a lack of theoretical framework guiding the research. Carvalho, Verissimo and Nobre (2013) have recently explored psychogenic contributors, including dysfunctional cognitive and emotional factors during PGAD and non-PGAD women’s sexual activity, as potential contributors to the disorder. Their research needs to be further expanded to consider the implications of dysfunctional cognitive and emotional factors not only during sexual activity, but also in everyday life.

\section{Purpose of the Study}

The purpose of this study was to fill a significant gap in the literature by providing a better understanding of significant predictors of distress in women with PGAD and moderators of said distress such as erotophobia-erotophilia and the nature of partner support. Leiblum and Chivers (2007) theorized heightened awareness of one’s physiological senses such as acknowledgment of genital arousal may spark a negative psychological response, increase anxiety levels, cue the sympathetic nervous system to increase activity, and ultimately increase focus on the genital arousal. The two 
researchers noted that anxiety disorders are reported at a higher frequency among those with PGAD who have higher distress rates than those who are not distressed.

As defined by Fisher, Byrne, White and Kelley (1988), erotophobia-erotophilia "involves the disposition to respond to sexual cues with predictable degrees of negativeto-positive affect and evaluations” (pp. 146-147). When examined on a continuum, those on the negative (erotophobic) end tend to exhibit more avoidance responses to sexual cues and behaviors, while those on the positive (erotophilic) end tend to exhibit more acceptance or approach responses. I sought to determine if anxiety would be the strongest predictor of distress, and if erotophobia-erotophilia and/or negative or poor emotional, instrumental (assistance with tasks), or informational (advice) partner support would moderate underlying anxiety, depression, and/or stress associated with distress in women with PGAD. Such findings may assist clinicians with psychological treatment approaches to reducing distress in women with PGAD.

\section{Theoretical Framework}

The theoretical framework for this study was the biopsychosocial model. George Engel (1980) made a strong case for use of this model in the fields of medicine, psychiatry, and psychology. He described the benefits of approaching the patient as a whole, utilizing a systems methodology of mind, body, and social influences. Engel articulated that the bottom-line goal for the patient is to relieve and alleviate distress, and proposed that in order to achieve this goal the healthcare provider must consider all angles of the problem being presented, whether medical, psychiatric, or psychological. With the biopsychosocial model, biological, psychological, and social influences should 
all be considered when formulating hypotheses to etiology of illness or conditions presented.

In the area of sexual dysfunction, this model is decidedly relevant given that psychosocial factors often play a large role alongside possible biological concerns. McCabe et al. (2010) specifically addressed the need to include a biopsychosocial perspective when examining sexual dysfunction, particularly given how anxiety, depression, sexual and nonsexual partner concerns, and life stresses can vastly impact sexual function. With respect to distress in PGAD, the biopsychosocial model can be used to examine the role of biological factors such as medical conditions and the aforementioned psychological predictors on levels of distress, as well as to examine if these predictors are moderated by social factors such as partner support or psychosocial factors such as erotophobia-erotophilia.

\section{Research Questions and Hypotheses}

Research Question 1. Will anxiety be the strongest predictor of distress among the following three predictors: anxiety, stress, or depression?

$H_{0} 1$ : Anxiety will not account for the most variance of the three predictors of distress in women with PGAD.

$H_{1} 1$ : Anxiety will account for the most variance of the three predictors of distress in women with PGAD.

Research Question 2. Does erotophobia-erotophilia moderate the relationship between the strongest predictor (anxiety, stress, or depression) and level of distress? 
$H_{0}$ 2: Level of erotophobia-erotophilia will not moderate the relationship between the most significant predictor and level of distress in women with PGAD.

$H_{1}$ 2: Level of erotophobia-erotophilia will moderate the relationship between the most significant predictor and level of distress in women with PGAD.

Research Questions 3. Does the nature of perceived partner support moderate the relationship between the strongest predictor (anxiety, stress, or depression) and level of distress for PGAD women with partners?

$H_{0} 3$ : Nature of perceived partner support will not moderate the relationship between the most significant predictor and level of distress in women with PGAD.

$H_{1} 3$ : Nature of perceived partner support will moderate the relationship between the most significant predictor and level of distress in women with PGAD.

Research Questions 4. Is there a difference in distress levels for PGAD women with partners compared to PGAD women without partners?

$H_{0} 4$ : Level of distress will not be different for PGAD women without partners than for PGAD women with partners.

$H_{1} 4$ : Level of distress will be different for PGAD women without partners than for PGAD women with partners.

\section{Nature of Study}

This was a quantitative study. Quantitative research is characterized by examining associations between variables to test theories (Creswell, 2014), which was the primary focus of this study. The quantitative approach allows researchers to analyze 
numerical variables captured via instruments such as survey questionnaires, and enables them to use appropriate statistical procedures and reporting.

The independent variables in this study were anxiety, depression, and stress. The dependent variable was the level of distress in women with PGAD. Moderating variables were the level of erotophobia-erotophilia and the nature of perceived partner support.

From a review of the literature, I identified the variables for this study which I then collected and measured via the survey method of collection and analysis. I used the Female Sexual Distress Scale-Revised (FSDS-R), a validated 13-item questionnaire, to assess sexually-related distress in the absence of an available, validated PGAD-related distress scale (see Fisher, Davis, Yarber, \& Davis, 2013).

The instrument I used to assess depression, anxiety, and stress was the validated 21-item Depression Anxiety Stress Scales (DASS-21). There are seven items for each of the three factors. This instrument, using Cronbach’s alpha, has reliabilities of .88 for the Depression scale items, .82 for the Anxiety scale items, and .90 for the Stress scale items (Henry \& Crawford, 2005).

I used the Sexual Opinion Survey, a validated scale with adequate test-retest reliability (Davis, Yarber, Bauserman, Schreer, \& Davis, 1998; Fisher, et al., 1988), to assess erotophobia-erotophilia. The Actually Received Support, a subscale of the Berlin Social Support Scales with a Cronbach alpha's of .83, was utilized to examine the nature of perceived partner support (Schulz \& Schwarzer, 2003).

Demographic variables captured included age, race, education level, relationship status, sexual orientation, employment status, income level, and religion/spirituality. 
Basic gynecological and medical history including pre- or post-menopausal, number of pregnancies, number of children, method of childbirth (cesarean or vaginal), history of overactive bladder, history of restless leg syndrome, history of Tarlov cysts, history of pudendal neuropathy, and history of sexually transmitted infections (STIs) were also collected. I also collected data regarding onset of PGAD symptoms, exploratory distress and embarrassment levels, and whether a clinician was visited regarding PGAD and how satisfied the participant has been with clinician support. Other than a minimum age requirement of 18 years and meeting the criteria for PGAD, no further inclusion or exclusion criteria were dependent upon demographic variables.

\section{Operational Definitions}

The following operational definitions are provided for clarification:

Anxiety: Excessive worry or fear that may be combined with physiological and somatic symptoms such as irritability, inability to or difficulty in concentrating, fatigue, restlessness, tension, sweating, stomach upset, shakiness, and dry mouth (Wilson, Mannix, Oko-osi, \& Revicki, 2015).

Depression: A mood disorder encompassing such symptoms as sadness, lessened interest or pleasure, fatigue, appetite and/or weight changes, difficulty sleeping or falling asleep, difficulty concentrating, feelings of worthlessness, hopelessness, guilt, and sometimes suicidal thoughts or attempts (Fried \& Nesse, 2015).

Erotophilia: A personality trait reflecting a positive attitude or disposition regarding sexuality (Macapagal \& Janssen, 2011). 
Erotophobia: A personality trait reflecting a negative attitude or disposition regarding sexuality (Macapagal \& Janssen, 2011).

Perceived partner support: The perception that an individual is cared for and assisted by their partner, and the partner is available to provide emotional, instrumental, and informational sustenance when needed (Girme, Overall \& Simpson, 2015; Overall, Fletcher \& Simpson, 2010).

Stress: “An appraisal that one's coping resources are inadequate in relation to the level of demands, prompting emotional, physiological, and behavioral changes that ultimately endanger well-being” (Amirkhan, Urizar Jr., \& Clark, 2015, p. 985).

\section{Assumptions, Limitations, and Delimitations}

In this study, I assumed that (a) the self-report measures would yield valid data, (b) level of distress is a key component of PGAD, and (c) the instruments used for data collection would provide accurate measure of the variables under study. Limitations of the study included a small sample size from a convenience sample of one medical facility specializing in sexual medicine and one online PGAD support group, reducing the ability for generalization to an overall population. Delimitation of the study, resultant from time and financial constraints, included purposely choosing to use only the convenience sample as opposed to searching nationwide for other women with PGAD.

\section{Significance}

This project is important because there are currently limited data in the literature showing women with documented PGAD as having a potential association between level of distress and anxiety, depression, or stress. Further there is no literature in which 
researchers have addressed the potential moderation of distress by the level of erotophobia-erotophilia and/or the nature of perceived partner support. In a recent study, Carvalho et al. (2013) examined emotional determinants such as anxiety and depression in women with PGAD; however, their study limited this examination to beliefs, thoughts, and emotions during sexual intercourse. Data from my research can be used to better understand the relationship between these phenomena without restricting the study to emotional determinants during sexual intercourse, and may indicate novel therapeutic modalities such as mindfulness or cognitive centered approaches based on expanded characterization of the disorder. Given my use of the FSDS-R to measure distress, this study also provides a better understanding of sexual distress among women with PGAD, a phenomenon that has not been assessed in prior PGAD studies.

PGAD is unfortunately associated with embarrassment and shame for many of the women suffering from the disorder (Leiblum, 2008). This can theoretically lead to women being less likely to report their symptoms to healthcare providers (Leiblum, 2009), and increased distress and/or the development of suicidal ideation (Eibye \& Jensen, 2014). With so little research of the disorder in existence, women suffering from PGAD need more facts and clarity from well-conducted research in order to inform both the general public and clinicians about the nature of this disorder, the distress it brings, and viable treatment options. By better characterizing the condition and exploring potential contributors to distress, we may be able to identify risk factors and triggers, a key step in the development of psychological-based therapies for these women. 


\section{Summary}

In this chapter, I have outlined the definition of PGAD along with the background of its recent introduction into the literature of sexual dysfunction. I have provided theories regarding the low prevalence of information available to date, highlighting the need to fill the extraordinary gap in the literature. Furthermore, the chapter has included an explanation of the purpose of the study, the theoretical framework upon which the study was built, and a summary the quantitative methodology used.

Chapter 2 begins with an extensive overview of PGAD including the history, etiological theories, associated distress, and treatments attempted to date. Secondly, the chapter includes an explanation of how depression, anxiety, and stress have played a role in various areas of sexual dysfunction. Finally, I discuss erotophobia-erotophilia and why this phenomenon may be a contributor to distress levels in women with PGAD, and provide an examination of the role of perceived partner support in illness. 
Chapter 2: Literature Review

\section{Search Strategy}

Given the limited amount of research in the area of PGAD, I reviewed literature published from 1995 up until present time. Databases I used to search for relevant literature included: Walden University’s Thoreau, PsycINFO and PsycTESTS, SCOPUS, OVID, MEDLINE, PubMed, ScienceDirect, and Google Scholar.

Key search terms and phrases I used as search criteria included persistent genital arousal disorder, persistent sexual arousal syndrome, PSAS, PGAD, restless genital syndrome, erotophobia, erotophilia, female sexual dysfunction, female sexual distress, social support, depression, stress, anxiety, distress, and sexual distress.

\section{Persistent Genital Arousal Disorder}

\section{History}

PGAD first appeared in the literature in 2001 when Leiblum and Nathan (2001) published a seminal paper describing a rare condition they termed persistent sexual arousal syndrome. In 2003 an international committee of sexuality experts assembled to recommend revisions in the classification of women's sexual dysfunctions, and PSAS was provisionally included and characterized as:

- Spontaneous, intrusive, and unwanted genital arousal (e.g., tingling, throbbing, pulsating), which occurs in the absence of sexual interest and desire.

- Any awareness of subjective arousal is typically but not invariably unpleasant.

- The arousal is unrelieved by one or more orgasms and the feelings of arousal persists for hours or days (Basson et. al, 2003). 
Over the next few years, Leiblum and a small group of collaborators attempted to further explore this phenomenon using (novel at the time) internet methods to reach a larger sample of this rare phenomenon, culminating in recruitment of a sizable sample of women with PSAS (Leiblum, et al. 2005). The researchers used an in-depth, detailed questionnaire to obtain information from the subjects $(N=103), 56 \%$ of whom were subsequently characterized as having met criteria for PSAS. In a subsequent study, Leiblum et al. (2007) recruited several other cohorts of women using online methodologies for data collection, and determined the nomenclature should be changed to the current persistent genital arousal disorder (PGAD). Leiblum et al. surmised this to be a better reflection of the finding that this is a condition of genital rather than sexual arousal because sexual arousal and/or sexual desire and thoughts are often, if not always, absent, and that sexual behaviors such as masturbation or sex with a partner were used to relieve the genital arousal sensations. Subsequently, five criteria were established as the diagnostic characteristics of PGAD, including:

1. Spontaneous, persistent genital arousal such as clitoral tingling, throbbing, congestion, pain, pressure or spontaneous orgasm that occurs for an extended period of time, often hours, days, weeks or months.

2. The arousal does not resolve or only temporarily resolves upon orgasm(s).

3. The arousal is unrelated to sexual desire.

4. The arousal may be triggered by non-sexual stimuli (e.g. riding in a car, riding a bicycle), sexual stimuli, or no stimuli at all. 
5. The arousal is intrusive, unwanted, bothersome, and causes distress

(Komisaruk \& Lee, 2012; Leiblum et. al 2007).

Seeking to further understand the phenomenon, Leiblum and Seehuus (2009) next assessed overall sexual function using the validated Female Sexual Function index (Rosen et. al, 2000) to compare a cohort of women with PGAD to a cohort of women with female sexual arousal (FSA) disorder and normal controls, and found there was no evidence of any "hypersexuality” or excessive sexual desire (Carvalho et al., 2015) in the PGAD cohort. In fact, the researchers found that these women did not meet cutoff scores for sexual dysfunction at all. Leiblum and Seehuus indicated that this might be due, in part, to women with PGAD associating their arousal symptoms with desire. During this time, work by Chivers et al. (2004) into the nature of sexual arousal in non-PGAD women led to an understanding that genital arousal in women was a poorly and probably misunderstood phenomenon. Subsequently, Leiblum et al. (2007) further surveyed 388 women online, some of whom indicated all five PGAD symptoms and some of whom did not. The women with full-symptom PGAD reported greater levels of distress and symptom severity than those with non-full symptom PGAD, bringing the researchers to the conclusion that "there is a cohort of women who regularly, and intermittently, experience noticeable sensations of genital arousal that are pleasurable and even reassuring” (p. 686). There are other women, too, who experience more persistent feelings of genital arousal that is experienced as mildly distracting but not especially unwelcome or bothersome, as noted in a study conducted by Garvey, West, Latch, Leiblum and Goldmeier (2009), where, out of 96 women who completed the study, two 
women reported having four of the five criteria for PGAD; however, they were not overly distressed by their symptoms, while three other women reported three of the five criteria for PGAD and experienced no distress or intrusiveness. In one study, researchers noted that women with PGAD exhibited more negative thoughts during sexual intercourse such as those of sexual abuse or failure, more negative emotions during intercourse such as hostility or depression, and more conservative sexual beliefs such as sexual desire and pleasure being sinful, than did women without PGAD (Carvalho et al., 2013). Although the aforementioned factors have been associated with PGAD, it is uncertain as to whether they are causal or a result of PGAD. Finally, there are also women who describe more continuous, intense, and persistent genital arousal that is reported to be extremely distracting, distressing, and worrisome. It is this last group of symptoms that has been labeled as PGAD (Leiblum \& Chivers, 2007).

Eventually, other researchers began to study this phenomenon, most notably Marcel Waldinger, a Dutch neuropsychiatrist. Waldinger et al. (2009) reported on a small ( $N=18$ ) cohort of women with PGAD who also presented with symptoms of overactive bladder (OAB) and restless leg syndrome ([RLS]; Waldinger et. al, 2009; Waldinger \& Schweitzer, 2009). Based on this cohort of combined conditions, the researchers proposed a neurologic mechanism for the disorder, which they termed restless genital syndrome (Waldinger et. al, 2009). The researchers used electroencephalograms (EEG) and magnetic resonance imaging (MRI) of the brain and pelvis to gain insight into potential biological pathologies for PGAD. For any women who had abnormalities on these tests, transvaginal ultrasonongraphy and repeat MRI with contrast added were 
performed to investigate potential causes of PGAD. They found that some women had pelvic and ovarian varices (dilated veins); however, the researchers concluded that no explicit pathology could be determined for the PGAD symptoms experienced by the 18 women who participated, thus suggesting there may be no specific biological etiology. In more recent literature, researchers have continued to review the preceding body of evidence, as well as to report new cases and putative therapies (Rosenbaum, 2010; Ramic, 2013); however, there has been a paucity of original, systematic, prospectively designed research into the phenomenon. Recent contributions have been made by researchers such as Carvalho et al. (2013) who have examined the correlates of PGAD with cognitive and affective aspects as well as with potential psychological moderators such as narcissism and neuroticism. They found that, although not necessarily causal or a result of PGAD, women with PGAD symptoms had higher levels of sexual conservatism and more negative affective states (e.g. low excitement, low interest, distressed) and thoughts (e.g., lack of affection from partner) during sexual activity than women without PGAD. Yildirim, Hacioglu, Essizoglu and Kucukparlak (2012) raised the concern of underreporting or misdiagnosis of PGAD in three case studies of Muslim women previously diagnosed with obsessive-compulsive disorders. The diagnosis was based on the grounds of bathing rituals practiced by these women upon sexuality-related behaviors such as intercourse, orgasm, and even menstruation. Because these women were bathing multiple times per day due to their persistent arousal and orgasms, and were hesitant to discuss their concerns due to fear of being seen as hypersexual, they were instead treated for OCD and subsequently received no relief. Fear, guilt, shame, and embarrassment 
have been cited across the literature as the potential reasons behind low reporting levels of PGAD (Markos \& Dinsmore, 2013; Leiblum, 2008; Mahoney \& Zarate, 2007).

\section{Etiology}

The etiology of PGAD remains a mystery. Graziottin (2004) identified the potential for neural problems as an underlying cause of genital arousal disorders. Others, such as Leiblum (2009), have concurred with this theory, suggesting that pelvic nerve hypersensitivity or brain lesion anomaly may play a role.

As clinicians began to grow more aware of the phenomenon, case reports as well as putative therapies began to appear in the literature (Goldmeier \& Leiblum, 2008). Cases associated with medication-induced changes such as antidepressant use and withdrawal (Leiblum \& Goldmier, 2008), vascular malformations (Bedell et al., 2014), psychological factors (Leiblum et al., 2005), and the presence of Tarlov cysts (defined as sacral region cysts; Komisarik \& Lee, 2014; Eibye \& Jensen, 2014) have been reported, challenging the search for an underlying or unifying theory of etiology. As an example of the variety of etiological possibilities, Pink, Rancourt, and Gordon (2014) reported on a group of 15 women who presented at a pain clinic for pelvic and/or genital pain, bladderrelated pain, headache, and other non-cancerous pain conditions with concomitant symptoms of PGAD. One of the participants was noted to have a Tarlov cyst, one had adenomyosis (endometrial tissue growing in muscle outside the uterus), and one had nonspecific foci (white spots) of the brain noted on MRI. Physical examination of the women showed nine positive Q-tip tests (a cotton swab placed in the urethra to evaluate urethral mobility), four with pain on palpation or digital exam, three with clitoral pain, 
one with clitoral swelling, one with hyperalgesia (increased sensitivity to pain), and one with hypoesthesia (reduced sensation to stimuli, particularly touch). A third of the women had been taking SNRIs either currently and/or in the past, while a quarter of the women were on SSRIs in the past. Approximately 50\% of the women in this cohort had taken tricyclic antidepressants in the past. Five of the women reported having restless leg syndrome, nine had a history of depression, and six suffered from anxiety. Vincenzo Puppo (2014) argued, in a letter to the editor of The Journal of Obstetrics and Gynaecology Canada, against the Pink et al. report, suggesting that PGAD should not be the nomenclature used for this cluster of symptoms, but rather female priapism. He indicated that the symptoms are more in line with that of female priapism, a condition consisting of prolonged erection of the clitoris unassociated with sexual arousal and causing pain and swelling in the surrounding areas as well. Pink et al. (2014) offered an extensive rebuttal to his letter, sufficiently explaining why his argument does not hold for several reasons, including that the most substantiating symptom of PGAD is arousal, whereas priapism is unrelated to arousal. Pink et al. also pointed out that Puppo failed to associate the chronicity of PGAD to priapism, whereby PGAD often occurs for months, while priapism generally occurs for hours.

Pelvic Congestion and Varices. In an earlier paper, Graziottin (2004) ascertained that PGAD could be described as a painful congestion of the genitalia accompanied by persistent arousal not relieved by orgasm, resulting in distress and removing all feelings of pleasure. Thorne and Stuckey (2007) presented a case report of a 62-year-old woman diagnosed with pelvic congestion syndrome, a chronic condition caused by varicose veins 
in the pelvic region. She also presented with ovarian vein incompetencies and complained of PGAD. Several varices (dilation of veins) were noted in her pelvic and genital regions upon ultrasound and MRI examinations. Upon endovascular coil embolization (blockage of blood flow) of the ovarian vein, the patient experienced approximately 70\% relief of persistent arousal symptoms. These researchers concurred that pelvic congestion syndrome should be considered a high possibility among etiologies being suggested.

Krapf and Goldstein (2013) recently reported on two cases whereby PGAD was concomitantly present in a 25-year-old woman and a 22-year-old woman who both appeared to suffer from a condition known as hypermobility-type Ehlers-Danios syndrome, a connective tissue disorder causing chronic musculoskeletal and joint pain. The authors identified the condition as "a collagen disorder that may have gynecologic manifestations” (p. 2347). In each of these women, stark labial edema (swelling of the labia) was present along with bladder pressure upon intercourse in the 25-year-old woman, and pain and sensitivity upon intercourse in the 22-year-old woman. A third case of PGAD being concomitant with Ehlers-Danlos syndrome was noted by Pink, Rancourt, and Gordon (2014) in their aforementioned cohort study above. Because these are the only three cases of this reported in the literature, etiological generalization would be inappropriate at this time.

In 2008, Marcel Waldinger et al. conducted an extensive study of 18 Dutch women diagnosed with PGAD. The researchers used brain and pelvic MRI, electroencephalogram (EEG), transvaginal ultrasound (TVUS), and blood samplings to 
look for any marked findings or similarities among the women. Over half of the women were noted to have pelvic varices ranging from mild, moderate to severe, with the majority of those being mild to moderate. Interestingly, several of the women also were noted to have had leg varices.

Tarlov Cysts. Tarlov cysts, otherwise known as perineural or sacral cysts, are fluid-filled cysts that form on the dorsal roots of the sacral region (S1 - S4) of the spinal canal, typically around S2 and S3 (Komisaruk \& Lee, 2012). As mentioned by Komisaruk and Lee (2012), the dorsal roots are associated with the sensory pudendal and pelvic nerves, which intertwine with both internal and external genitalia and have the potential to promote atypical genital sensations, bringing rise to the hypothesis that Tarlov Cysts could be implicated as an etiology for PGAD.

Komisaruk and Lee (2012) conducted a study involving the use of a PGAD support group to recruit women who had prior MRIs. The researchers evaluated the MRIs of 18 women who self-reported PGAD (a limitation of the study) and noted that 12 of the 18 women had notable Tarlov cysts. Five of the 12 women had one cyst, six of the 12 women had two cysts, and one of the 12 women had three cysts. The researchers noted that in the general population, Tarlov cysts appear in $1.2-9 \%$ of the population and thereby concluded that Tarlov cysts could be considered a conceivable etiology for PGAD. Eibye and Jensen (2014) confirmed a Tarlov cyst present in a case report of a 31year-old female presenting with PGAD among other comorbidities including depression, anxiety and somatization. 
Restless Genital Syndrome. In 2009, Marcel Waldinger and Dave Schweitzer conducted a second review on the 18 Dutch women mentioned above. In their "Part 2" review of the data, they noted that 12 (67\%) of the 18 women not only suffered from PGAD, but also experienced restless leg syndrome (RLS) and overactive bladder with a frequency in micturition (the urge for emptying their bladders), which led to subsequent brief relief of PGAD symptoms. RLS is defined as "a neurological disorder characterized by throbbing, pulling, creeping, or other unpleasant sensations in the legs and an uncontrollable, and sometimes overwhelming, urge to move them” (National Institute of Neurological Disorders and Stroke, 2010). The researchers found that several of the women reported their RLS onset occurring at the same time as or prior to their PGAD onset. Waldinger and Schweitzer (2009) noted the similarities between RLS and PGAD such as, but not limited to, the uncomfortable sensations, the urge to alleviate the sensations through movement or rubbing the inflicted area, and varices in the inflicted regions (pelvis and legs). They further reported a "clustering” of these symptoms and theorized they could be lumped into a condition they deemed restless genital syndrome (RGS), a more complex syndrome that encompasses RLS, PGAD, overactive bladder and urethra hypersensitivity. Markos and Dinsmore (2013) concurred with the notation of similarities between PGAD and RGS and went on to suggest that these similarities can also be found in vulvodynia, a condition involving chronic pain of the external genital region. In their 2013 manuscript, Markos and Dinsmore suggested that PGAD and RGS be considered subtypes of vulvodynia and argued that it should be reclassified as a pain disorder as opposed to a sexual dysfunction, which appears to be based upon their 
interpretation of the similarity in symptoms and the evolving definition of vulvodynia over the past few decades.

Waldinger continued his research with Schweiter as well as Venema and van Gils (2009), utilizing the data from 12 of the 18 PGAD patients above and adding another 11 new PGAD women to conduct further testing and analysis of his newly defined RGS. The results of this study concluded that, in fact, all the women examined presented varices in the pelvic region and seven of them had varices in the legs. Eighteen of the 23 women reported RLS and sixteen reported overactive bladder. These figures give further merit to the theory of a clustering of symptoms grouped together as restless genital syndrome.

In this same study, the researchers also used a new approach in their examinations called genital tactile mapping testing (GTM) to conduct pressure point touch responses in the hopes of mapping the specific genital region involved in RGS. Using a cotton swab on the skin, the researchers touched upon several pressure points with fixed pressure on or around the genitals, the pubic bone, the groins, the anal area and the perineum to test for tactile sensations. Various trigger points were noted among the women, including the area above the pubic bone in 11 of the women and ranging moderately across all of the other aforementioned areas resulting in hypersensitivity from light to moderate pressure, as well as repeated intense orgasm in three of the women. For four of the women, the clitoris was the only notable trigger point to spark RGS.

Pudendal Neuropathy. It is speculated that the pudendal nerve, originating from the pudendal plexus in the S2-S4 sacral region, and the dorsal nerve of the clitoris (DNC) 
play a role in RGS and PGAD. The pudendal nerves extends from the pudendal plexus to the pudendal canal, perineum and gluteal regions, and has been previously identified as contributing to bladder issues, pelvic/genital/rectal pain and sexual dysfunction (Possover \& Forman, 2012), giving feasibility to the theory of its involvement in RGS and PGAD. The DNC function, however, is not quite clear, though in the Waldinger et. al (2009) study it was found to induce pre-orgasmic and orgasmic sensations albeit a lack of sexual desire.

Medication-Induced Changes. Researchers have presented case studies in the past noting patients who have developed PGAD upon use, change, or discontinuation of prescription medications such as selective serotonin reuptake inhibitors or serotonin and norepinephrine reuptake inhibitors. In particular, Battaglia and Venturoli (2009) shared a case study of a 29-year-old woman presenting with PGAD as well as uncontrollable orgasms for two years upon the use of Trazadone for depression/anxiety. Increased sexual arousal and spontaneous orgasm began one month into Trazadone use. The medication was abruptly stopped after three months; however, the persistent arousal and spontaneous orgasms remained, although slightly decreased. Clitoral and vaginal congestion were noted by the patient during examination by the researchers who suggested further study into clitoral vascularization in order to better understand what may be occurring in these cases. Leiblum et al. (2007) also suggested that, in some rare instances, clitoral priapism (an occurrence involving "increased intracavernosal pressure or vascular outflow problems” (p. 1359)) may be camouflaged as PGAD. 
In another case study, Mahoney and Zarate (2007) discussed a 32-year-old woman who reported increased clitoral sensation and PGAD-like symptoms upon an increase in venlafaxine dosage. Elimination of symptoms occurred for this particular patient upon dose reduction. In further research, using results from an online survey of 364 women conducted in 2005, Leiblum and Goldmeier (2008) found six women who indicated that symptoms of PGAD began upon the usage or discontinuation of SSRIs. Goldmeier, Bell, and Richardson (2006) hypothesized that discontinuation of SSRIs may trigger PGAD in women via withdrawal syndrome phenomena. This includes the potential for high production levels of atrial natriuretic peptide (ANP), which is related to vasodilatation and increased vascular endothelial permeability, potentially resulting in vasocongestion and edema in the genital region.

In the aforementioned Eibye and Jensen (2014) case, the woman had been previously diagnosed with dependent personality disorder and was prescribed a variety of different antidepressants and anti-anxiety medications. Per the patient, her PGAD symptoms began upon cessation of paroxetine, an SSRI used in the treatment of her psychological distress. Paroxetine was restarted in the hopes of alleviating the symptoms; however, complete relief was not obtained and, in fact, worsened upon the addition of agomelatine, another antidepressant.

Psychological Correlates. In 2007, Leiblum et al. sought not only to explore the medical and pharmacological correlates of PGAD, but also the psychological correlates. They noted that women with PGAD were "more likely than women who only met some of the criteria to report depression, anxiety, panic attacks, and certain obsessive- 
compulsive symptoms such as monitoring their physical sensations.” The authors hypothesized that for some women with PGAD, "psychological factors, namely anxiety, reinforce, exacerbate and maintain PGAD.” Gadit (2013) presented a case report of a 54year-old woman suffering from comorbidities of PGAD, including anxiety, obsessivecompulsive behavior, and non-specific stress syndrome, all of which were reported prior to the patient receiving prescribed medications. Stress induced psychological changes have also been attributed to exacerbating PGAD (Leiblum, 2009). Hamilton and Meston (2013) implicated chronic stress, including distraction in women with high chronic stress, as a contributing factor to sexual dysfunction. Leiblum and Goldmeier (2008) as well as Pink, Rancourt and Gordon (2014) have noted depression as a noteworthy correlate in women experiencing sexual dysfunction. Leiblum and Goldmeier pointed out that in some studies in upwards of $70 \%$ of women who suffered from major depression reported difficulties with sexual function. Pink, Rancourt and Gordon noted over half (60\%) of their study cohort of 15 women had symptoms of depression. Anxiety was also noted in six (40\%) of their 15 participants. While these factors have been associated with PGAD, no cause or effect relationship has been determined.

Other Associations. Other researchers have looked at further associations of PGAD factors. A case report by Anzellotti et al. (2010) centered on PGAD being associated with an epileptic focus in a 40-year-old female. This female had no notable abnormalities on prior MRI and EEG screenings. Upon administration of an antiepileptic pharmacological treatment, the woman’s PGAD symptoms subsided. 


\section{Distress}

One of the five diagnostic criteria of PGAD is that the arousal is intrusive, unwanted, bothersome and causes distress. Leiblum and Chivers (2007) noted varying levels of distress in women with PGAD and suggested three overall categories of arousal in women including: a) unprompted genital arousal sensations that are perceived as somewhat pleasurable; b) chronic arousal that is perceived as distracting but not distressful or unwelcome; c) chronic, intense arousal that is distressful, unwelcome and bothersome. The researchers provided statements from two women from an online survey who had several symptoms of PGAD without the distress, and appear to both accept and welcome their symptoms. One woman went so far as to state she hopes her symptoms remain into her senior years. These are followed by contrasting statements from others such as that of a 38-year-old woman indicating both the need to masturbate multiple times per day to relieve her unbearable genital sensations starting in early childhood years and the shame and guilt she felt in doing so. With so little research existing on PGAD overall, it is extremely difficult to find even minimal literature focusing solely on distress factors (none of which examine sexual distress) and the underlying influences for the variations in distress levels among PGAD women. The limited research available theorizes that negative appraisal, variability in intensity and/or length of the genital sensations, high cognitive awareness, and hypersensitivity to one's physical sensations resulting in anxiety could be implicated as distress predictors (Carvalho et al., 2015; Leiblum \& Chivers, 2007; Leiblum et al., (2007). Leiblum and Chivers (2007) also noted that psychological attributes may be the etiological culprits in 
cases where physical attributes cannot be ascertained. They proposed the following model:

1) "Women detect a spontaneous genital sensation;

2) They appraise their sensation or response as negative or inappropriate;

3) They experience anxiety subsequent to their appraisal;

4) The anxiety subsequently increases sympathetic nervous system activity, enhancing their perceptions of and attention to their genital sensations”; (p. 368).

Social and cultural constraints regarding women’s sexuality including sex negativity may contribute to feelings of shame, guilt, embarrassment and worry contributing to higher levels of distress, including sexual distress (Leiblum \& Chivers, 2007; Leiblum et al., 2005). Most recently, Carvalho et al. (2015) examined personality, sexual beliefs and relationship adjustment as contributors to PGAD. They found that neuroticism, as a personality factor, did not have a significant impact on distress in PGAD, while low openness did, and that dyadic adjustment and sexually conservative beliefs were significant predictors of distress. Again, these factors are only found to be associated with PGAD and not causal or consequential therefore limiting generalization.

\section{Treatment}

Definitive treatments for PGAD are still uncertain. Different treatment approaches have been utilized dependent upon the hypothesized pathology of the PGAD. In some instances, cognitive behavioral therapy or counseling has been utilized to assist with negative cognitions and stress (Leiblum \& Goldmeier, 2008; Leiblum, Seehus, 
Goldmeier, \& Brown, 2007; Gadit, 2013). In other cases, patients were treated by targeting Tarlov cysts with nerve or epidural blocks, or given anti-seizure medication, mood stabilizers (Komisaruk \& Lee, 2012) or antidepressants, though this remains controversial as SNRIs and SSRIs have been noted as potential triggers of PGAD (Pink, Rancourt, \& Gordon, 2014; Gadit, 2013).

Korda, Pfaus, Kellner and Goldstein (2009) presented a case report of a bipolar (type I) female with PGAD who was treated with electro-convulsive therapy (ECT), resulting in temporary relief of symptoms, slowly relapsing over time. Gadit reported on a patient who was offered ECT but was uncomfortable proceeding with the treatment. Waldinger et al. (2010) discussed a case report of a 77-year-old woman who underwent a clitoridectomy with moderate relief from spontaneous orgasm but little relief from arousal symptoms and sensations. In another study by Waldinger et al. (2009), two women who presented with RGS were given transcutaneous electrical nerve stimulation (TENS) with positive results. One of the women had a 90\% reduction in spontaneous orgasm as well as immediate relief from other arousal symptoms, while the second woman obtained complete relief of genital symptoms over a two-month period.

Philippsohn and Kruger (2012) presented case studies of two women: a 36-yearold treated with duloxetine and a 41-year-old treated with pregabalin, an SNRI and a gamma-Aminobutyric acid analog anticonvulsant and analgesic often used in neuropathy, respectively. The duloxetine treated woman obtained full remission from her symptoms while the pregabalin treated woman received substantial but not full relief. On the other 
hand, Gadit (2013) reported a patient was taking Citalopram, Clonazepam and Zopiclone with little effect on PGAD symptoms.

In Waldinger's and Schweitzer's (2009) Dutch study, the authors noted the participants had obtained various treatments over time with little or no improvement, including pelvic floor exercises, pharmacological treatment including antidepressants and/or cyproterone acetate and psychotherapy. Three women were prescribed pramiprexol, a non-ergoline dopamine agonist used in RLS and Parkinson's, with no improvement. Three women were given tramadol, an opioid used for pain, resulting in temporary (approximately 4 hours) reduction of symptoms for two of the women. Three women were given oxazepam, a benzodiazepine used for anxiety and depression, with reports of symptom reduction in two and overall relief in one. Finally, two menopausal women were given hormone replacement therapy (HRT) with symptom improvement in one woman but not the other.

Rosenbaum (2010) provided a case report of a 27-year-old pregnant woman who received pelvic floor manual therapy focusing on the pudendal nerve. Per the patient, the symptoms of PGAD significantly reduced after approximately one week of therapy. Upon 10 and 14 week follow up phone contacts, the patient indicated complete resolution of symptoms.

In one case, a 71-year-old woman, with comorbidity of Parkinson’s disease (onset ten years prior to PGAD onset) received nine sessions of hypnotherapy as treatment approach to her PGAD symptoms (Elkins, Ramsey, \& Yu, 2014). Her symptoms first appeared shortly after a Mirapex (pramiprexole) dose increase. The patient had 
previously used Lidocaine and ice baths for her symptoms, but with minimal temporary effect. Upon completion of the nine hypnotherapy sessions, the woman showed marked improvement in her PGAD symptoms.

As many of these treatment approaches consist of only single case reports or small participant numbers, it is difficult to surmise or generalize consistent, appropriate, effective treatment patterns across the population. Further research on PGAD treatments is direly needed.

\section{Depression, Anxiety and Stress in Sexual Dysfunction}

Research over the last decades has shown consistent associations between sexual dysfunction and affective states, depression, and anxiety (Lewis et al, 2010, Laumann, Paik, \& Rosen, 1999; Shifren, Monz, Russo, Segreti,\& Johannes, 2008). Unfortunately, almost all of these studies have significant methodological problems and have been largely cross-sectional. Thus, despite the plethora of correlations noted, to date almost no longitudinal or experimental studies have been undertaken that would demonstrate potential causality between the occurrence of sexual dysfunction and these affective disorders. Although this study will also have the limitation of being cross-sectional due to time and financial constraints, the study is expected to add a significant contribution to the limited PGAD research available.

Lovibond and Lovibond (1995) examined the DASS (Depression Anxiety Stress Scales) scales to determine overlap potential among depression, anxiety and stress. While they noted that some overlap does occur, particularly between anxiety and stress, the scale does portray notable differences among the three constructs. Depression was 
marked by low or loss of self-esteem, loss of incentive, and a low perception of life goal achievability among the more commonly addressed symptoms of sadness and worthlessness. Anxiety was marked by nervousness, shakiness, dry mouth, panic, fear and worry. Stress was marked by difficulty with relaxing, nervousness (overlap with anxiety), agitation, irritability and impatience.

Anxiety. From Freud's earliest writings, anxiety played a significant role in early psychodynamic formulations of sexual dysfunction and later became the foundation for the etiological concepts of sex therapy established by Masters and Johnson and Helen Singer Kaplan. Indeed, Kaplan (1995) asserted that sexually-related anxiety was "the 'final' common pathway via which various biological and psychosocial factors influence the modulation and adjustment” of human sexuality (p. 19). More recently, studies have repeatedly found that individuals with sexual dysfunction exhibit heightened levels of anxiety, supporting the possibility of a central role of anxiety in the subjective experience and maintenance of sexual concerns.

The central role of anxiety historically reported by sex therapists (Kaplan, 1995; Rosen \& Leiblum, 1995) has been challenged by a number of sophisticated laboratory studies aimed at unraveling the sequence of cognitive-affective processes during sexual arousal in dysfunctional and functional men and, to a lesser extent, women. Contrary to the clinical studies' findings for an inhibition effect of anxiety, the laboratory evidence indicated that anxiety (as induced in the lab setting) either facilitates or does not affect sexual arousal in sexually functional subjects. The evidence for sexually dysfunctional subjects is more mixed (Mitchell, DiBartolo, Brown, \& Barlow, 1998). 
Anxiety researcher David Barlow offered a theoretical model explaining why anxiety may operate differentially in subjects with and without sexual dysfunction. His model emphasizes the role of cognitive interference; in general, he suggests that what distinguishes functional from dysfunctional responding is a difference in selective attention and distractibility (Barlow, 1986). What has long been described clinically as performance anxiety, or fear of inadequacy or "spectatoring” can all be seen as a situation-specific cognitive activity that distracts dysfunctional individuals from processing of stimuli in a positive sexual context (Althof et al., 2005). Experimental psychophysiology studies in women suggest the relationship between anxiety and sexual function is mixed, with the suggestion that it is more negative than enhancing (Meston \& Bradford, 2007). Leiblum and Chivers (2007) addressed both the hyperactive physiological and psychological effects anxiety can produce within the body, thus providing conceptual support for the hypothesis that anxiety will be the strongest predictor of PGAD in this study.

In summary, psychophysiology studies examining the relationship between anxiety, distraction, general sympathetic activation, and sexual response have demonstrated that anxiety is not universally disruptive to sexual functioning in women (Wiegel, Scepkowski, \& Barlow, 2006). Importantly, results have suggested that the relationship between anxiety and sexuality - particularly in women - is complex, with evidence suggesting context, level and the nature of anxiety and its history are important determinants. As an example, moderate anxiety and perceived "safe” settings may heighten sexual arousal, while higher levels of anxiety, loss of personal control, or a 
history of abuse seem to impair sexual functioning (Meston et al., 2006; Lemieus \& Byers, 2008).

Depression. Although sexual arousal disorders have not been as extensively investigated in women as in men, it is generally accepted that there is a bi-directional impact of depressive symptomatology and sexual function. Clinical treatment guidelines recommend that women with depressive symptomatology be screened for sexual dysfunction and vice versa, that women presenting with sexual dysfunction be assessed for the presence of depression (Basson et al., 2004; Althof et al., 2005; McCabe et al., 2010).

In his landmark study of sexual concerns in the United States, Laumann et al. (1999) reported a latent class analysis demonstrating that depressive symptoms and/or stress predicted a 4.6 times greater likelihood of arousal disorder in women. A study in the UK utilizing similar methodology by Dunn, Croft and Hackett (1999) found that in a community sample of 615 women, self-reported problems with arousal were six times more likely in women with, versus those without, depression.

Cyranowski et al. (2004a) noted that arousal was significantly lower in a sample of 914 women with recurrent major depressive disorder compared to women with no history of depressive disorder. This was so even when controlling for current depression scores, psychotropic medication use, comorbid anxiety or substance abuse; whereas women who had only a single episode of major depression did not show such an effect. Another study by Cyranowski et al. (2004b) showed that of 68 women experiencing at least their 2nd episode of major depression and not using psychotropic medication at 
study entry, $41 \%$ reported that in the past 30 days they had not had normal lubrication throughout sexual relations. Even after interpersonal therapy, an assessment at 12months showed that $38 \%$ of women still reported disturbances in arousal, suggesting that sexual symptoms comorbid with depression do not easily disappear, even when the depression is treated and/or in remission. Similar findings were reported by Kennedy, Dickens, Eisfeld, and Bagsby (1999), in which similar rates of dysfunction with arousal was noted in a sample of 102 depressed women, 50\% of whom reported less sexual arousal overall, and 40\% reporting difficulty in achieving adequate vaginal lubrication.

In women, only one experimental study was found involving depression and arousal. In this repeated measures psychophysiology experiment, 56 women with normal sexual function were classified as either depressive or not and vaginal photoplethysmography was taken as an objective measure of sexual arousal; self-report measures of arousal were also taken. Although there were no significant differences between women classified as depressed or not depressed on objective or subjective measures of arousal, the findings suggested that holding negative thoughts or affect that are experienced before sexual activity commences can decrease sexual responding, even when positive affect is introduced later (Kuffel \& Heiman, 2006).

Stress. The potential role of stress in sexual dysfunction is far more difficult to assess, because stress can be conceptualized in many different ways. In their study of older American sexuality, Laumann et al. (2008) noted “sexual problems among the elderly seem more of a response to stressors in multiple domains of life, from physical health to features of the intimate relationship” (p. 2309). Similarly, broad findings were 
noted by Shifren, Monz, Russo, Segreti, and Johannes (2008) in their large $(N=31,581)$ survey of women. They found that poor self-assessed health, low education level, depression, anxiety, thyroid conditions, and urinary incontinence all correlated with distressing sexuality. Thus, while stress appears to be implicated negatively with sexual function, a specific role that can be parsed out does not present itself given the broad nature of the construct and the variability to what may constitute stressors to an individual. The literature does not reflect it having ever been tested in a prospective manner as to do so would require a complex longitudinal methodology, e.g. recruitment of a cohort with low stress, anxiety and depressive symptoms at baseline in order to ascertain the relationship between the variables over time. Alternatively, although more experimental in nature, a cohort of subjects could be recruited and their subjective and objective sexual responses assessed in response to sexual stimuli in the presence or absence of co-administered stress stimuli.

\section{Erotophobia and Erotophilia}

As indicated by Macapagal and Janssen (2011), sex involves a wide range of emotions, sentiments, reactions, and connotations, some positive and some negative. Sexuality experts have noted that all individuals can be categorized as having an erotophobic or erotophilic disposition (Furnham \& Hirandani, 2009; Fisher, Byrne, \& White, 1983). Erotophobia falls at the negative end of the erotophobia-erotophilia spectrum eliciting negative responses to sexual stimuli, while erotophilia falls at the positive end, igniting positive responses to sexual stimuli (Furnham \& Hirandani, 2009; Fisher et al., 1988). Byrne and Kelley (1986) contended that those individuals who fall 
somewhere in the middle of the spectrum have a greater tendency to lean more toward erotophilia as they gain more gratifying sexual experiences over time.

Furnham and Hiranandani (2009) indicated that sex guilt mediates negative emotions such as anxiety and avoidance reactions in erotophobes in response to sexual stimuli. Such sex guilt may consist of anxiety-inducing emotions when thoughts or feelings related to sexual behavior arise in the erotophobe (Furnham \& Hirandani, 2009). Hall, Hogue and Guo (2014) contended that erotophobic individuals have higher sex guilt than erotophilic individuals. Researchers also note that sex can elicit not only guilt, avoidance and anxiety, but also disgust, annoyance, threat (Cherner \& Reissing, 2013) and distress for some individuals, including those who are considered erotophobic or even those suffering from sexual dysfunction (Macapagal \& Janssen, 2011; Wiegel, Scepkowski, \& Barlow, 2007). In the aforementioned case report by Rosenbaum (2010), the 27-year-old pregnant woman suffering from PGAD expressed feelings of concern regarding her newly compulsive need to masturbate to relieve her symptoms because of her religiosity, role identity and self-image. Per the case report, the woman indicated she attributed arousal to sexual feelings, which should only be experienced through sexual relations with her spouse, and therefore was distressed by her “overly sexual” symptoms (p. 1307).

An earlier study by Helweg-Larsen and Howell (2000) regarding the processing of sexually related material supported the theory of avoidance in that it showed women who were considered erotophobic, or sex negative, were more likely to avoid sexual information, and that erotophobia appears to interfere with centrally-routed processing of 
sexual information. Smith, Hedges, Gerrard, and Gibbons (1996) found similar results in their study in that sexually related cognition and concepts may be altered dependent upon one's tendency to be considered either erotophobic or erotophilic.

Other researchers such as Evans (2000) have noted that myths relating to sex contribute to erotophobia. Myths such as masturbation causing harm or women having sex or swimming during menstruation leading to negative outcomes and detrimental effects on one's livelihood can stimulate erotophobic attitudes in individuals. According to Evans (2000), these myths can be unintentionally substantiated by erotophobic clinicians who avoid discussing sexually related concerns with clients and patients, a term noted by Evans as institutional erotophobia. Institutional erotophobia combined with individual erotophobia may theoretically provide one explanation for the low prevalence of PGAD cases reported to date, particularly when stigma is factored in.

Stigma, specifically with respect to sexually related illness, or perceived sexually related illness such as PGAD, can have ubiquitous influence on individuals inflicted with these disorders. In a study on the prevalence of sexual dysfunction in a population of 417 male and female teachers, Adegunloye and Gwabachi Ezeoke (2011) found that 53\% of respondents who had indicated they had some form of sexual dysfunction (i.e. painful sex, female orgasm disorder, low or no desire, premature ejaculation) did not seek treatment. Of those 53\%, fear of stigma accounted for $26 \%$ of the reasoning for not seeking treatment. Smith and Nave (2007) indicated social rejection scores of erotophobes towards fictitious patients with sexually transmitted infections were higher in their study than those of fictitious patients with non-sexually transmitted illnesses. 
These stigmas not only contribute to social rejection, but have also been shown to contribute to depression, anxiety, and stress among people suffering from sexually related illness (Smith \& Nave, 2007; Fife \& Wright, 2000; Barth, et al., 2002; Crandall \& Moriarty, 1995) and sexual dysfunction.

Manifestation of erotophobia in sexually related illness or perceived sexually related illness such as PGAD has been noted in the literature. Bergeron, Rosen and Morin (2011) referenced a randomized vestibulodynia (pelvic pain) treatment trial whereby level of erotophobia contributed to $27 \%$ of surgical outcome variance. Women who were more erotophobic, based on their results of the Sexual Opinion Survey, responded less favorably to vestibular surgery than women who were less erotophobic. The researchers suggested that erotophobic beliefs could ultimately contribute to continuance of pain behavior and avoidance of sex even after pathophysiologic symptoms had been addressed (Bergeron, Khalife, Glazer \& Binik, 2008). Other researchers such as Lucena, Mukhopadhyay and Morris (2015) concurred with the recognition of erotophobic manifestation in sexually related illness, noting that high levels of erotophobia have also been found in women with dyspareunia (pain with intercourse). Manifestations such as these provide the foundation and reasoning for the hypothesis that erotophobia could be a predictor or moderator in cases of PGAD. For example, women with erotophobic dispositions might experience genital sensations as suggestive that they are somehow “dirty" or "shameful”, contributing to a state of anxiety and / or distress. Erotophilic individuals may experience the same level of genital throbbing or fullness but experience it cognitively as either a neutral or even mildly pleasurable sensation. 


\section{Social Support System}

Social support, and more specifically partner support, is an important factor to consider when discussing predictors, correlates, moderators and mediators of the everincreasing abundance of illnesses faced today. Peloquin, Bigras, Brassard and Godbout (2014) emphasized the importance of recognizing partner support as crucial with respect to the role it plays in both sexual health and overall well-being. These researchers, in particular, examined whether the perception of partner support would be a protective factor in correlations between psychosexual adjustment and several aspects of sexuality, including assertiveness, anxiety, avoidance, self-esteem under the realm of attachment theory. Their findings noted that people with high attachment avoidance levels exhibited more erotophobic reactions to sexually related stimuli and were more uncomfortable regarding sexually related partner interactions than those with lower attachment avoidance levels. Early research by Dewitte (2012) noted similar findings, as did that of Girme, Overall, Simpson and Fletcher (2015) who indicated that those with high attachment avoidance can react defensively when approached with partner support. Interestingly, Peloquin et al. (2014) also noted that, in those with high attachment avoidance levels, sexual anxiety was higher when partner support was perceived as high. The researchers speculated that when partner support was perceived as high in high attachment avoidance individuals, poor psychosexual adjustment was amplified, perhaps elucidated by the intensified discomfort level of closeness and intimacy in these individuals. This concurred with Girme et al. assessment above regarding the potential for high avoidance individuals to react negatively to partner support. Brock and 
Lawrence (2014; 2009) went one step further, first by acknowledging that not all support is adequate support, and second by defining two dimensions of inadequate partner support: underprovision, or lack of adequate support and overprovision, or too much support. Subsequently, they indicated that perceived overprovision of the wrong kind of partner support, such as too much advice, can actually be more detrimental to a relationship than perceived underprovision of support. This is perhaps due to the imbalance of support level each partner requires, meaning, for example that the receiving partner may not respond favorably to an "overdose” of support and experience it as psychologically or emotionally unsettling.

In further research, Peloquin, Brassard, Lafontaine and Shaver (2014) noted that partner support provided, whereby the partner attempted to solve all the problems and minimized the opportunity for the affected partner to solve problems, correlated to lower sexual satisfaction in women. This coincided with Brock’s and Lawrence's (2014) research above regarding the relationship of overprovisioning and satisfaction.

Other researchers, however, held contrary views. Simpson, Winterheld, Rholes and Orina (2007) supported the notion that for some high attachment avoidant individuals, partner support is well-received and meritoriously calms the recipient, particularly when the level of support is high. Rholes et al. (2011) similarly concluded that high levels of support in high attachment individuals ultimately brought about less depressive symptoms.

Low avoidance individuals tend to receive all levels of support in a positive, accepting manner (Girme et al., 2015; Collins \& Feeney, 2004). This population tends to 
harbor more trust in partner support and are less likely to ascertain the level of support equates to level of acceptance or rejection. Interestingly, Bolger and Amarel (2007) as well as Gleason, Iida, Shrout and Bolder (2008) have suggested that high levels of partner support may actually interfere with resilience in low avoidance individuals by challenging their coping competencies.

Researchers such as Bergeron, Rosen and Morin (2011) pointed out that in genital related disorders such as dyspareunia and vaginismus, relationship support and response are often ignored. Research can be more readily found regarding partner support in illnesses such as cancer. Sawin (2012) described the experiences of non-supportive partner relationships in older women with breast cancer, indicating that silence and shutting down was prevalent in the relationship, acting as defense mechanisms for both relationship participants. Kinsinger, Laurenceau, Carver and Antoni (2011), in their study of perceived partner support in breast cancer patients, indicated that women who perceived their partner to be exhibiting supportive behaviors were more positively influenced with respect to relationship satisfaction and sexual adjustment. As these researchers point out, perceived support has been shown to play a vital role in relationships inflicted with illness and therefore should be examined more closely by researchers across a myriad of fields of study.

\section{Summary}

Persistent genital arousal disorder has become a matter of discussion among clinicians and researchers within the past two decades. Sandra Leiblum was a pioneer in bringing the disorder to our attention and shedding light on its distressing symptoms 
(Leiblum \& Nathan, 2001). Over the past few years, other researchers have continued the quest to uncover the pathology underlying the disorder. Those such as Graziottin (2004), Goldmeier and Leiblum (2008), Bedell et al. (2014), Leiblum et al. (2005), Komisarik and Lee (2014), Eibye and Jensen (2014), and Pink, Rancourt and Gordon (2014) have unearthed a broad potential of etiologies such as Tarlov Cysts, pelvic varices, neural concerns, psychological factors, or previous SSRI/SNRI use. Waldinger et al. (2010, 2009) expanded the etiological theories to suggest PGAD may be part of a grouping of symptoms they collectively deem restless genital syndrome. Treatments currently remain limited, emphasizing the importance of further research needed on the disorder.

With so little research available on PGAD, the gaps in the literature remain large. Psychological factors have been considered, including the premise that anxiety may play a role; however, the literature remains deprived of significant psychological predictors and moderators of PGAD. Two of the more puzzling questions that remain regarding PGAD center on causality and nature of the distress that women experience. A number of hypotheses regarding potential causality have been generated, including Tarlov cysts, pelvic congestion, restless leg/genital syndrome and pudendal neuropathy.

Chapter 2 illustrated the small amount of empirical support available for these hypotheses. Of note however, most of these have been based on case-reports or post-hoc analyses of small samples. No one has yet attempted to ascertain utilizing open-ended items to determine what, if anything, women with PGAD associate with its development / onset. Regarding distress, it is important to note there are no valid, reliable instruments 
that have been developed to specifically address the distress that women with PGAD experience, leaving researchers with an imperfect understanding of this important phenomenon. 
Chapter 3: Research Method

\section{Introduction}

The purpose of this study was to provide a broader understanding of significant psychological correlates of distress in women with PGAD, specifically the potential contribution of depression, anxiety, and stress, as well as the impact of erotophobiaerotophilia and the nature of perceived partner support as moderators. In this chapter, I explain the methodology and procedural approach that I used in the study. Research design, rationale for the design, sampling procedures, instrumentation and operational procedures will also be illuminated.

\section{Research Design and Rationale}

PGAD is still in the early stages of discovery and understanding. Little is known about the pathology of this disorder, or why some women have higher distress levels than others. Psychological factors have been implicated as possible etiologies in prior research; however, no formal studies have been done to hone in on psychological contributors to distress, and there is no clear clinical understanding of what factors might modulate the distress. My goal in this study was to do just that: examine the role of three psychological correlates (depression, anxiety, stress) on levels of distress and the potential for erotophobia-erotophilia or positive/negative levels of perceived partner support to moderate those levels of distress.

A quantitative, cross-sectional, correlational, non-experimental, survey methodological approach was used with a convenience sample of women with PGAD. I recruited the participants from those individuals who have sought consultation and/or 
treatment from San Diego Sexual Medicine (SDSM) in San Diego, California, and from an online Facebook PGAD support group. I chose the clinical site because of its stellar reputation as a major sexual health clinic, along with its history of working with women who have PGAD and the willingness of the staff to work with up and coming researchers in the field of sexual health. I chose the online support group as a means to recruit an adequate number of women with PGAD, considering the small population who report the disorder. The addition of the support group increased the potential for recruiting women who had not yet sought treatment for PGAD, thereby increasing heterogeneity and reducing bias towards women who have sought treatment.

As previously noted the quantitative approach allows for the analysis of numerical variables captured via instruments such as survey questionnaires and allows for the utilization of appropriate statistical procedures and reporting. Correlational methodology provides for the measurement of predictability and relationships among variables; however, this method should not be confused with causal relationships (Creswell, 2008). Using a cross-sectional survey design allows for the collection of data at a given time point in a more economical and timely manner than a longitudinal framework (Creswell, 2014). With so little research currently available on PGAD, I determined that a crosssectional study with previously unstudied moderator variables could provide a significant contribution to the literature. 


\section{Methodology}

\section{Population}

The dissertation study recruited female participants meeting the five criteria for PGAD from two sources: the office of Dr. Irwin Goldstein at SDSM in San Diego, California, and an online private Facebook PGAD Support Group for Men and Women. While the women I recruited from SDSM had sought consultation and/or treatment from SDSM, they may physically be located throughout the globe, as are the women in the support group. Because this disorder is currently considered rare, potentially due to underreporting, the target population was low.

SDSM, which opened its doors in 2007, collects a variety of demographic data for their patients, including email addresses. Patients have agreed to contact from SDSM via email. Aggregated patient records from this institution indicate that approximately 250 (+/- 5\%) adult women are receiving or have received treatment or advice for PGAD from Dr. Goldstein since 2007 and continue to suffer from PGAD. I recruited 50 women meeting the inclusion criteria from the SDSM convenience sample via targeted emails by site staff. To keep the identities of participants confidential, I provided the study site with a letter (see Appendix B) along with the survey link to send to potential participants via email requesting their voluntary participation in the online study. I had no access to any medical charts. Once recruited into the study, participants completed a series of self-report questionnaires via Survey Monkey, an online survey center. A reminder/follow-up email was sent to potential participants 7 days after the original email thanking those who already completed the 
survey and reminding those who had not to please consider participating, followed by a final reminder at day 14.

For the participants in the online Facebook PGAD support group consisting of 131 female participants at the time of the study, I provided, via an online posting to the group, an invitation for survey participation (see Appendix C), an explanation of what the survey is and who may be eligible, and a link to the survey, including the informed consent. A reminder/follow-up post regarding voluntary participation was posted in the support group 7 days after the original email, followed by a second/final reminder 14 days after the original post.

\section{Sampling}

To determine the statistically appropriate sample size, I conducted a power analysis using G*Power 3.1 statistical software (see Faul et al., 2009). A medium effect size (0.30) was considered to be appropriate under the rationale that there was little empirical research to guide me to the ultimate effect size I should have been aiming for. Using the Test Family setting in $\mathrm{G}^{*}$ Power of $t$-tests, and the Type of Analysis set to $a$ priori, an effect size of 0.30 , and a conventional alpha level of .05, the G*Power calculator, with power of .80 , indicated a required sample size 64 . I chose this method over others such as prediction models (e.g. logistic regression) because the underlying function of the study was to detect correlations in PGAD and anxiety, depression, and stress with erotophobia-erotophilia and perceived partner support as moderators of these factors, and it was not to predict these factors as a cause or result of PGAD. 


\section{Data Collection Process}

I collected data from participants via an email/posted link to an online survey located on the Survey Monkey website. The survey consisted of multiple questionnaires including: the FSDS-R (Deragotis et al. 2008; Fisher et al., 2013), the DASS-21 (Henry \& Crawford, 2005), the Sexual Opinion survey (Davis, Yarber, Bauserman, Schreer, \& Davis, 1998; Fisher, et. al, 1988), and the Actually Received Support, a subscale of the Berlin Social Support Scales (Schulz \& Schwarzer, 2003), as well as a questionnaire that eliciting demographic and gynecological information.

The format of the survey was as follows: (a) an invitation to participate in the survey, (b) an explanation of the survey's purpose, (c) a description of what the survey entailed, (d) an explanation of the voluntary nature of the study and the risks and benefits of participation, (e) a confidentiality statement, (f) my contact information should any questions or concerns have arisen, (g) informed consent, and (h) a description of inclusion and exclusion criteria. These items were followed by a collection of demographic information including age, race, education, relationship status, income level, religion, spirituality, and sexual orientation. The following gynecological/medical questions were collected to assess their prevalence in women with PGAD for notation of potential biological pathology, as I noted in Chapter 1: pre- or post-menopausal, number of pregnancies, number of children, method of childbirth, history of overactive bladder (a yes/no question), history of restless leg syndrome (a yes/no question), history of Tarlov cysts (a yes/no question), history of pudendal neuropathy (a yes/no question), and history of sexually transmitted infections (a yes/no question). I collected history of 
SSRI and SNRI use, asking if these were taken "currently" and/or "in the past." Length of time the participant had PGAD symptoms and exploratory distress and embarrassment levels was collected, along with whether or not the participant had seen a healthcare provide regarding PGAD symptoms, how satisfied they were with the care received, and if they related their symptoms to any specific event.

The remaining items included all questionnaires mentioned above. The order of flow was as follows:

1. The FSDS-R.

2. The DASS-21.

3. The Sexual Opinion Survey.

4. The Actually Received Support subscale.

The final items at the end of the survey were two open-ended questions including:

1. Please describe in detail how PGAD has affected your life.

2. What aspects of PGAD do you feel contribute most to your distress? Inclusion criteria included:

1. Women age 18 and over who meet criteria for PGAD defined as the following (Waldinger \& Schweitzer, 2008):
a. Involuntary genital arousal that persists for an extended period of time.
b. The arousal does not dissipate upon orgasm.
c. The arousal is not related to sexual desire.
d. The arousal is intrusive and unwanted.
e. The arousal is distressful and / or bothersome. 
2. PGAD has been present for minimum of six months.

3. Ability to read, understand and provide informed consent.

Exclusion criteria included:

1. Inability to read and understand English.

\section{Instrumentation and Operational Constructs}

\section{Dependent Variable -Distress}

Distress associated with PGAD, the dependent variable, was measured using the FSDS-R (Deragotis et al., 2008; Fisher et al., 2013). This is due in part to the lack of an available PGAD distress scale. The FSDS-R is a validated 13-item, Likert-type questionnaire with responses ranging from 0 (Never) to 4 (Always), with higher scores equating to higher distress. An example from the questionnaire is: "How often did you feel guilty about your sexual difficulties?” Test-retest reliability for the FSDS-R showed greater than 0.74 for the intraclass correlation coefficient, and Cronbach's alpha of greater than 0.86 for internal consistency (Derogatis, et al., 2008). A copy of the scale can be found in Appendix A.

\section{Independent Variables - Depression, Anxiety, and Stress}

I assessed independent variables using a multilinear regression analysis. These variables included depression, anxiety, and stress, and I measured them using the validated, 21-item Likert-type DASS-21 (Henry \& Crawford, 2005). Responses range from 0 (Never) to 3 (Almost Always) and provided a separate score for each of the three factors under study. Scores indicate Normal, Mild, Moderate, Severe, or Extremely Severe levels of depression, anxiety, and stress. An example of a question is: "I found it 
hard to wind down.” Reliability for this instrument, using Cronbach’s alpha, is .88 for the Depression scale items, .82 for the Anxiety scale items, and .90 for the Stress scale items (Henry \& Crawford, 2005). A copy of the scale can be found Appendix A. Moderating Variables - Erotophobia/Erotophilia and Perceived Partner Support

I used moderation analysis to test the effects of the level of erotophobiaerotophilia and the nature of perceived partner support in women with a partner as moderating variables. A Mann-Whitney test was conducted to compare women with partners and women without. Assessment involved the determination of any increase or decrease in the established effects of depression, anxiety, and stress on PGAD distress. The Sexual Opinion Survey (SOS), a validated 21-item Likert-type scale with acceptable test-retest reliability with Cronbach’s alpha of 0.90 (Davis, Yarber, Bauserman, Schreer, \& Davis, 1998; Fisher et. al, 1988; Gilbert \& Gamache, 1984) was the instrument I used to assess erotophobia-erotophilia. An example question is: "If people thought I was interested in oral sex I would be embarrassed.” Responses range from Strongly Agree to Strongly Disagree. Negative (low) scores demonstrate erotophobia, while positive (high) scores demonstrate erotophilia. Scores for the SOS range from 0-126 upon calculation, using the addition of +67 in the scoring method to eliminate negative scores for simplification of scoring interpretation by keeping all scores positive (see Fisher et al., 1988). A copy of the scale can be found Appendix A.

I used the Actually Received Support, a Likert-type 14-item subscale of the Berlin Social Support Scales to examine the nature of perceived partner support for women with a partner (Schulz \& Schwarzer, 2003). This scale was skipped for those women who did 
not have a partner. An example of a question on the scale is: "The person comforted me when I was feeling bad.” Responses range from 1 (Strongly Disagree) to 4 (Strongly Agree). The scale consists of both positive (11) and negative (3) questions, resulting in negative question scores having to be reversed for questions 3, 4, and 5 . Higher scores demonstrate positive support received, while lower scores demonstrate less or lack of positive support. Reliability and validity have been established in several studies, indicating a Cronbach’s alpha for internal consistency and reliability of .83 (Schulz \& Schwarzer, 2003). A copy of the scale can be found Appendix A.

\section{Summary of Scales}

I chose the scales listed above based on their validity and extensive use in research. The length of each scale is adequate for sufficient data capture while short enough to keep survey fatigue at a minimum and encourage full completion by participants. All scale items combined equate to 69 questions, in addition to demographic, gynecological information and open-ended questions.

\section{Demographics and Gynecological Information}

Each participant was requested to complete demographic and gynecological information in order to categorize participants and capture prevalence of these variables among women with PGAD. Descriptive tables are presented in chapter 4 to show how many women fall into each of the categories.

\section{Open-ended Questions}

Although I used a validated measure of sexually-related distress in this study, its use does not suggest that sexually-related distress is the only, or even the most salient, 
aspect of the personal distress these women experience. In order to generate possible themes for hypothesis generation I asked a total of three open-ended questions in the survey, including:

o If participant answered "yes" to association of PGAD with a specific event, please specify event.

o Please describe in detail how PGAD has affected your life.

o What aspects of PGAD do you feel contribute most to your distress?

A significant number of comments were provided by the participants. I did not conduct a thematic analysis on these comments in this dissertation. These questions were intended for information gathering items to be coded and analyzed in future qualitative publications. Samples of comments with minimal discussion are presented later in this dissertation to be used as illustrative reference points for discussion in chapter 5 .

\section{Research Questions, Hypotheses, and Statistical Analysis Methodology}

\section{Data Screening and Cleaning}

I collected and retrieved data by export from SurveyMonkey.com. I performed statistical analysis using SPSS version 21. Statistical significance was based on an alpha level of .05. I performed an analysis to determine the degree of missing data. For questionnaires, no data points were present where less than $5 \%$ of the data were missing; therefore, eliminating the need for imputation of the mean.

I analyzed several items study including whether or not anxiety, stress, and/or depression are significant correlates of distress; which of these three psychological factors proved to have the strongest relation to distress; and if the relationship between 
the level of PGAD distress and anxiety, stress and/or depression was moderated by level of erotophobia-erotophilia and/or the nature of perceived partner support.

\section{Research Questions and Hypothesis}

Research Question 1. Will anxiety be the strongest predictor of distress among the following three predictors: anxiety, stress, or depression?

$H_{0} 1$ : Anxiety will not account for the most variance of the three predictors of distress in women with PGAD.

$H_{1} 1$ : Anxiety will account for the most variance of the three predictors of distress in women with PGAD.

Analysis: To analyze whether or not anxiety, stress, and/or depression are significant correlates of distress in women with PGAD, a hierarchical multiple linear regression analysis was utilized (Frankfort-Nachmias \& Nachmias, 2008). Assumptions for this test include normal distribution in the population and a random sample of observations drawn from the population of women with PGAD with variable scores independent of other scores on the same variables. This test was utilized because there are more than three variables involved, with distress being the dependent variable and all others being independent variables.

Research Question 2. Does erotophobia-erotophilia moderate the relationship between the strongest predictor (anxiety, stress, or depression) and level of distress?

$H_{0} 2$ : Level of erotophobia-erotophilia will not moderate the relationship between the most significant predictor and level of distress in women with PGAD. 
$H_{1}$ 2: Level of erotophobia-erotophilia will moderate the relationship between the most significant predictor and level of distress in women with PGAD.

Analysis: To analyze whether the relationship of distress in women with PGAD and anxiety, stress and/or depression is moderated by level of erotophobia-erotophilia, a multiple regression analysis was utilized under the assumption of normal distribution in the population. Holmbeck (1997) indicates this test is optimal for testing moderated effects on continuous variables and is specifically designed to test the degree of linear relationship between predictor and moderator variables that may or may not exist in a sample using quantitative variables.

Research Questions 3. Does the nature of perceived partner support moderate the relationship between the strongest predictor (anxiety, stress, or depression) and level of distress for PGAD women with partners?

$H_{0} 3$ : Nature of perceived partner support will not moderate the relationship between the most significant predictor and level of distress in women with PGAD.

$H_{1} 3$ : Nature of perceived partner support will moderate the relationship between the most significant predictor and level of distress in women with PGAD. Analysis: To analyze whether the relationship of distress in women with PGAD and anxiety, stress and/or depression is moderated by the nature of perceived partner support, a multiple regression analysis (Field, 2013) was performed to assess the contribution of the moderator variable that has association to distress as described above for erotophobiaerotophilia. 
Research Questions 4. Is there a difference in distress levels for PGAD women with partners compared to PGAD women without partners?

$H_{0} 4$ : Level of distress will not be different for PGAD women without partners than for PGAD women with partners.

$H_{1} 4$ : Level of distress will be different for PGAD women without partners than for PGAD women with partners.

Analysis: To analyze whether PGAD women with partners will have different levels of distress than PGAD women without partners, a one-way ANOVA was conducted to compare the two groups.

\section{Threats to Validity}

Due to the cross-sectional approach to the study, external validity is limited with respect to generalizing from the study sample to the population. As a convenience sample was used, limitations of reflecting the overall PGAD population are a consideration. Due to the nature of the study, reluctance to answer sensitive questions could have contributed to missing information and small sample size. No conclusions regarding cause and effect can be presumed from this study.

\section{Ethical Considerations}

I sought and obtained IRB approval prior to study initiation. The IRB approval number is 02-22-16-0023616. Each participant was provided an online informed consent form when they clicked on the link to the study. This form included an explanation of the nature of the study, the risks and benefits associated with study participation, a confidentiality statement, a statement of consent and age verification, and contact 
information of the researcher. Each participant was made aware of their voluntary participation and that they could have withdrawn from the study at any time without consequence simply by closing and submitting the survey either completed or incomplete. Participants were made aware that no follow-up to the survey is required. Personal monetary rewards were not provided for study participation.

The identity of the participants remained confidential. Email addresses were maintained by the clinical site and only the primary researcher had additional email access. I obtained no identifying information (e.g. name, address, birth date). Potential risks of the study included those associated with negative emotions and experiences based on the sensitive nature of the study. I provided links to informational resources regarding depression and anxiety (http://www.apa.org/helpcenter/understandingdepression.aspx and http://www.apa.org/topics/anxiety/index.aspx) to participants at the end of the study and encouraged participants to call their primary care physician if they became distressed upon taking the survey. I had no conflicts of interest in conducting this study.

\section{Summary}

In chapter 3, I provided an outline of the research design and methodology for the study of sexual distress in women with PGAD. Sample selection strategy, data collection, instrumentation tools, and analysis procedures were defined, including justifications and assumptions. I provided research questions and hypotheses along with how each was analyzed. Finally, I described inclusion and exclusion criteria, examined 
ethical considerations, and provided a notation of where all items (instruments and informed consent) can be found. 
Chapter 4: Results

\section{Introduction}

This chapter contains the results of the data analyses of the completed surveys. The purpose of the study was to examine predictors (anxiety, depression, stress) and moderators (erotophobia-erotophilia and partner support) of distress in women with PGAD. I examined (a) whether or not anxiety would be the strongest predictor, (b) whether or not erotophobia-erotophilia moderated the relationship between the strongest predictor and the level of PGAD distress, (c) whether or not the nature of perceived partner support moderated the relationship between the strongest predictor and the level of distress in women with PGAD, and (d) whether or not there is a difference in distress levels for women with PGAD with partners compared to women with PGAD without partners.

\section{Participant Demographic and Medical Information}

Seventy-three female participants agreed to participate in the online survey. Of the 73 responses received, 22 were excluded because of not meeting all five criteria for PGAD. Of the 51 remaining participants, 21 (41.2\%) were clients of SDSM, while 30 (58.8\%) were participants of an online PGAD support group. Response rates were 42\% (22 out of 50) for the SDSM group, and 22.9\% (30 out of 131) for the online support group. I conducted a one-way ANOVA on the dependent variable (FSDS-R scores) to compare the two groups of respondents from SDSM and the online support group. The ANOVA was not statistically significant, $F(1,49)=.577, p=.45$, indicating no significant difference between the two groups with respect to the dependent variable; 
therefore, the groups were combined for analysis of the research questions. Similarly, I conducted ANOVAs on the independent variables of anxiety scores, depression scores, and stress scores to compare the two groups of respondents from SDSM and the online support group. All three ANOVAs were not statistically significant $[F(1,50)=.266, p=$ $.814 ; F(1,50)=.056, p=.608 ; F(1,50)=2.66, p=.109$, respectively], indicating no significant difference between the two groups on these parameters.

The majority of participants (88.2\%) were located in the United States, while the remaining participants (11.8\%) were from Europe (i.e., England, Finland, Germany, Slovakia, and Sweden). The age range of the participants was from 21-years to 74-years old, with a mean age of $47(S D=13.37)$. The average amount of time respondents had had PGAD was 106 months, with the minimum number at 5 months and the maximum at 480 months (40 years).

No statistically significant correlation appeared between age and FSDS-R score ( $p$ $=$.76), as shown in Table 2. Similarly, a Pearson correlation was computed on duration of PGAD and FSDS-R score, and the result was not statistically significant $(p=.68)$. Participants reported varied backgrounds with regards to education, religion, spirituality, and sexuality. Table 1 illustrates the variability in responses. The majority of participants were either married (62.7\%) or in a relationship living with a partner (7.8\%), for a combined total of 70.5\%. Approximately half of the participants indicated working full- or part-time (27.5\% and $21.6 \%$ respectively, for a combined total of 49.1\%). Income levels were also varied, ranging from less than $\$ 25,000$ (8\%) to greater than 100,000 (14\%). The majority of participants were either exclusively or 
predominantly heterosexual both with respect to behavior (98\%) and fantasy (84.3\%) over the course of their lifetime.

Table 1

Demographic Characteristics of Study Sample

\begin{tabular}{|c|c|c|}
\hline Demographic & $N$ & $\%$ \\
\hline \multicolumn{3}{|l|}{ Association } \\
\hline San Diego Sexual Medicine & 21 & 41.2 \\
\hline Facebook $^{\mathrm{TM}}$ online support group & 30 & 58.8 \\
\hline \multicolumn{3}{|l|}{ Race } \\
\hline Caucasian & 47 & 92.2 \\
\hline Black or African-American & 2 & 3.9 \\
\hline Multiracial & 1 & 2.0 \\
\hline Other & 1 & 2.0 \\
\hline \multicolumn{3}{|l|}{ Education } \\
\hline High School Diploma & 11 & 21.6 \\
\hline Associate’s Degree & 7 & 13.7 \\
\hline Bachelor's Degree & 13 & 25.5 \\
\hline Master’s Degree & 9 & 17.6 \\
\hline Doctorate Degree & 4 & 7.8 \\
\hline Other & 7 & 13.7 \\
\hline \multicolumn{3}{|l|}{ Religious Affiliation } \\
\hline Christian & 27 & 52.9 \\
\hline Jewish & 2 & 3.9 \\
\hline Atheist / Agnostic & 6 & 11.8 \\
\hline No religious affiliation & 10 & 19.6 \\
\hline Other & 6 & 11.8 \\
\hline
\end{tabular}




\begin{tabular}{|c|c|c|}
\hline Demographic & $N$ & $\%$ \\
\hline \multicolumn{3}{|l|}{ Spirituality } \\
\hline Not at all Spiritual & 4 & 7.8 \\
\hline Slightly Spiritual & 9 & 17.6 \\
\hline Somewhat Spiritual & 12 & 23.5 \\
\hline Quite Spiritual & 18 & 35.3 \\
\hline Extremely Spiritual & 8 & 15.7 \\
\hline \multicolumn{3}{|l|}{ Employment } \\
\hline Full time employed & 14 & 27.5 \\
\hline Part time employed & 11 & 21.6 \\
\hline Student & 2 & 3.9 \\
\hline Retired & 7 & 13.7 \\
\hline Non-employed & 17 & 33.3 \\
\hline \multicolumn{3}{|l|}{ Household Income } \\
\hline$<\$ 25,000$ & 8 & 15.7 \\
\hline$\$ 25,001$ to 50,000 & 10 & 19.6 \\
\hline$\$ 50,001$ to 75,000 & 7 & 13.7 \\
\hline$\$ 75,001$ to 100,000 & 11 & 21.6 \\
\hline$>\$ 100,000$ & 14 & 27.5 \\
\hline Did not respond & 1 & 2.0 \\
\hline \multicolumn{3}{|l|}{ Relationship Status } \\
\hline Single & 8 & 15.7 \\
\hline Married & 32 & 62.7 \\
\hline Separated & 1 & 2.0 \\
\hline Divorced & 6 & 11.8 \\
\hline In a relationship, living with partner & 4 & 7.8 \\
\hline
\end{tabular}




\begin{tabular}{lcc}
\hline Demographic & $N$ & $\%$ \\
\hline Sexuality - Kinsey Scale -Fantasy & & \\
Exclusively heterosexual & 23 & 45.1 \\
Predominantly heterosexual, rare homosexual & 20 & 39.2 \\
Predominantly heterosexual, more than rare homosexual & 3 & 5.9 \\
Equally heterosexual/homosexual & 3 & 5.9 \\
Predominantly homosexual, more than rare heterosexual & 1 & 2.0 \\
Predominantly homosexual, rare heterosexual & 1 & 2.0 \\
Exclusively homosexual & 0 & 0.0 \\
Sexuality - Kinsey Scale - Behavior & & \\
Exclusively heterosexual & 35 & 68.6 \\
Predominantly heterosexual, rare homosexual & 15 & 29.4 \\
Predominantly heterosexual, more than rare homosexual & 1 & 2.0 \\
Equally heterosexual/homosexual & 0 & 0.0 \\
Predominantly homosexual, more than rare heterosexual & 0 & 0.0 \\
Predominantly homosexual, rare heterosexual & 0 & 0.0 \\
Exclusively homosexual & 0 & 0.0 \\
\end{tabular}


Table 2

Correlation Between Study Variables and Distress

\begin{tabular}{lc}
\hline Measure & FSDS-R
\end{tabular}

FSDS-R $^{1}$

Age

$-.04$

Duration of PGAD ${ }^{2}$

.06

Religion

.20

Spirituality

.13

Sexuality-fantasy

.13

Sexuality-behavior

.20

No. of Medical Diagnoses

$.36^{* *}$

No. of Pregnancies

$.35 *$

No. of Births

.25

Depression

$.51^{* *}$

Anxiety

$.28 *$

Stress

.25

Sexual Opinion Survey

.00

Partner Support

.07

${ }^{*} \mathrm{p}<.05 ;{ }^{* *} \mathrm{p}<.01 ;{ }^{1}$ Female Sexual Distress Scale-R; ${ }^{2}$ Persistent Genital Arousal Disorder 
I conducted bivariate correlations between sexual distress and the following demographic variables: age, religiosity, spirituality, sexual fantasy, sexual behavior, erotophobia-erotophilia, and partner support (see Table 2). None of these variables were found to have a correlation with sexual distress measured by the FSDS-R. Bivariate correlations were also conducted on sexual distress and medical diagnoses and gynecological history variables including total number of medical diagnoses, number of pregnancies and number of births (see Table 2). Total number of medical diagnoses and number of pregnancies were the only variables found to have a significant correlation with sexual distress measured by the FSDS-R. Pearson correlations were computed between erotophobia-erotophilia and FSDS-R, as well as partner support and FSDS-R. No statistically significant correlations were found between sexual distress measured by the FSDS-R and perceived partner support or the SOS (see Table 2).

Using sexual distress as the dependent variable, I conducted a series of ANOVAs on other demographic variables including race, $F(3,47)=1.44, p=.244$; education, $F(5$, $45)=.47, p=.794$; employment, $F(4,46)=5.15, p=.002$; income, $F(4,45)=1.25, p=$ .304 ; and religion, $F(4,46)=.552, p=.699$; as well as on the nominal gynecological variable menopausal status, $F(3,47)=.634, p=.597$. Employment status was the only variable of these to have a relationship to distress measured by the FSDS-R. Follow-up tests were conducted on employment status to evaluate the pairwise differences among the means. Dunnett's $C$ test was used, a test that does not assume equal variances, due to the small sample size (Green \& Salkind, 2011). There was a significant difference in the means between part-time employed and the non-employed groups, but no significant 
differences between the others. The non-employed group showed higher FSDS-R scores compared to the part-time employed group (see Table 3). Similarly, an ANOVA conducted on the non-employed group showed higher scores on all three subsets of the DASS-21 psychological measurements (depression, anxiety, and stress).

Table 3

Employment Descriptives

\begin{tabular}{lllll}
\hline Employment Status & $M^{1}-$ FSDS-R & $M-$ Depression & $M-$ Anxiety & $M-$ Stress \\
\hline Full-time & 35.57 & 6.14 & 4.50 & 9.79 \\
Part-time & 28.73 & 9.09 & 8.09 & 13.18 \\
Student & 45.00 & 14.00 & 4.00 & 14.50 \\
Retired & 37.71 & 5.57 & 5.00 & 9.29 \\
Non-employed & 48.47 & 14.53 & 10.82 & 15.24 \\
& & & &
\end{tabular}

Frequencies of medical diagnoses and gynecological history were performed and presented in Table 4. Several respondents reported more than one medical diagnosis (39.2\%). 
Table 4

Medical History

\begin{tabular}{|c|c|c|}
\hline & $N$ & $\%$ \\
\hline \multicolumn{3}{|l|}{ Ever been diagnosed with? } \\
\hline Overactive Bladder & 15 & 29.4 \\
\hline Restless Leg Syndrome & 12 & 23.5 \\
\hline Tarlov Cysts & 4 & 7.8 \\
\hline Pelvic Congestion Syndrome & 2 & 3.9 \\
\hline Pudendal Neuropathy & 16 & 31.4 \\
\hline Sexually Transmitted Infection & 16 & 31.4 \\
\hline \multicolumn{3}{|l|}{ Number of diagnoses from above } \\
\hline 0 & 15 & 29.4 \\
\hline 1 & 16 & 31.4 \\
\hline 2 & 11 & 21.6 \\
\hline 3 & 9 & 17.6 \\
\hline \multicolumn{3}{|l|}{$\begin{array}{l}\text { Gynecological History } \\
\text { Menopausal Status }\end{array}$} \\
\hline Pre-menopausal & 16 & 31.4 \\
\hline Peri-menopausal & 9 & 17.6 \\
\hline Post-menopausal & 21 & 41.2 \\
\hline Unknown & 5 & 9.8 \\
\hline
\end{tabular}




\begin{tabular}{lcc}
\hline & $\mathrm{N}$ & $\%$ \\
\hline Number of Pregnancies & 16 & 31.4 \\
0 & 7 & 13.7 \\
1 & 11 & 21.6 \\
2 & 8 & 15.7 \\
3 & 9 & 17.6 \\
4 or more & & \\
Number of Births & 19 & 37.3 \\
0 & 10 & 19.6 \\
1 & 11 & 21.6 \\
2 & 5 & 9.8 \\
3 & 19 & 11.8 \\
4 or more & 6 & 37.3 \\
Method of Birth & & \\
Vaginal & 59.2 \\
C-Section & 20 & 13.7 \\
Both & 7 & 9.8 \\
Not Applicable & 5 & \\
\hline & & \\
\hline & & \\
\hline & & \\
\hline
\end{tabular}


Antidepressant medication (SSRIs and others) use was assessed for current use, past use, and both. Of the 51 participants, 40 reported they had taken an SSRI or other antidepressant in the past, while 22 were currently taking at least one of these medications, and 18 indicated both current and past use (Table 5).

Table 5

SSRI and other Antidepressant Use

\begin{tabular}{lcc}
\hline Use of medication & $N$ & $\%$ \\
\hline Current use & 22 & 43.1 \\
Past use & 40 & 78.4 \\
Both current and past use & 18 & 35.3 \\
\end{tabular}

A majority of the women (58.8\%) who participated in the study indicated they have seen four or more healthcare providers regarding their PGAD symptoms, with less than one-third (27.4\%) indicating they are at least somewhat satisfied with the support they have received from their providers. In response to a non-validated question inquiring about PGAD symptom distress over the past six months, the majority of respondents indicated they were quite or extremely distressed (84.3\% combined total). Participants also indicated they were at least somewhat embarrassed about their PGAD symptoms (somewhat $=23.5 \%$; quite $=19 \%$, extremely $=9 \%$ ). Almost two-thirds of the participants (62.8\%) associated the onset of their PGAD symptoms with a specific event (i.e. - SSRI or other medication use, pregnancy, hysterectomy). 


\section{Predictors of Sexual Distress}

\section{Hypotheses - Research Question 1}

The null and alternative hypotheses for Research Question 1 were as follows:

Research Question 1. Will anxiety be the strongest predictor of distress among the following three predictors: anxiety, stress, or depression?

$H_{0} 1$ : Anxiety will not account for the most variance of the three predictors of distress in women with PGAD.

$H_{1} 1$ : Anxiety will account for the most variance of the three predictors of distress in women with PGAD.

I conducted data analysis using the SPSS 21 software package. Collinearity between the three predictor variables (anxiety, depression, stress) was assessed and confirmed based on the Variance Inflation Factor (VIF) statistics (anxiety: VIF = 2.38; depression: $V I F=2.51$; stress: $V I F=2.87)$.

Simple bivariate correlations between anxiety, depression, and stress were computed using Pearson's r. Statistically significant correlations occurred between anxiety and depression $(r=.69, \mathrm{p}<.01)$ and anxiety and stress $(r=.73, \mathrm{p}<.01)$, and stress and depression $(r=.75, \mathrm{p}<.01)$. There were also statistically significant correlations between the stress and age $(r=-.41, \mathrm{p}<.05)$ as well as depression and age ( $r$ $=-.35, \mathrm{p}<.05$ ). Sexual distress measured by the FSDS-R was significantly correlated to depression $(r=.51, \mathrm{p}=.00)$ and anxiety $(r=.28, \mathrm{p}=.044)$. Stress $(r=.25, \mathrm{p}=.083)$ was not statistically significant in correlation computations to sexual distress measured by the FSDS-R. Mean scores for FSDS-R and subsections of the DASS-21 are shown in Table 
6. Mean scores for all three psychological factors fell within the moderate range of severity per DASS-21 scoring measures (Henry \& Crawford, 2005).

Table 6

Means and Standard Deviations

\begin{tabular}{lcccc}
\hline Measure & Mean & SD & Possible Range & Obtained Range \\
\hline FSDS-R Distress $^{1}$ & 25.24 & 14.53 & $0-52$ & $0-52$ \\
DASS & & & & \\
DASS Anxiety & 9.80 & 5.82 & $0-21$ & $0-21$ \\
DASS Stress & 7.43 & 7.96 & $0-21$ & $0-21$ \\
Sexual Opinion Survey & 61 & 21.64 & $0-126$ & $2-21$ \\
BSSS $^{3}$ Partner Scale & 48 & 10.00 & $15-60$ & $0-97$
\end{tabular}

${ }^{1}$ Female Sexual Dysfunction Scale; ${ }^{2}$ Depression Anxiety Stress Scale; ${ }^{3}$ Berlin Social Support Scale.

I conducted a hierarchical multiple regression analysis to determine the strongest significant predictor of sexual distress, if any. I chose a hierarchical multiple regression based on research and theory in the literature that have demonstrated anxiety to be a significant contributor to distress in PGAD and also to allow for the possibility that anxiety is statistically not the best predictor (i.e. to allow for the opportunity to reject the hypothesis). Depression and stress were put into block 1, while anxiety was put into block 2 to determine if it added to the prediction over and above the prior two variables. Preliminary analyses were performed to ensure there was no violation of the assumption of normality, linearity and multicollinearity. A statistically significant regression equation was found, $F(2,48)=10.01, p<.01$. The $R^{2}$ for step 1 was .30 as it was for step 2 . 
Anxiety was excluded from the equation $(p=.97)$ while stress showed no statistical significance ( $p=.10)$, indicating that depression was the best and only statistically significant predictor of sexual distress measured by the FSDS-R, accounting for $30 \%$ of the variance, while anxiety did not contribute to the variance (Table 7). Therefore, I failed to reject null hypothesis.

Table 7

Hierarchical Regression

\begin{tabular}{lcccccc}
\hline Step 1 & $b$ & SE B & $\beta$ & $p$ & $R^{2}$ & $\Delta R^{2}$ \\
\hline Constant & 32.46 & 5.24 & -- & $<.01$ & .30 & .27 \\
Depression & 1.83 & .46 & .73 & $<.01$ & -- & -- \\
Stress & -.98 & .59 & -.30 & .10 & -- & -- \\
\hline Step 2 & & & & & & \\
\hline Constant & 32.50 & 5.41 & -- & .97 & .30 & .25 \\
Anxiety & .02 & .55 & .01 & .97 & -- & -- \\
\hline
\end{tabular}

Moderators of Sexual Distress

\section{Hypotheses - Research Questions 2 and 3}

The null and alternatives hypotheses for Research Question 2 The null and alternatives hypotheses for Research Question 2 were as follows:

Research Question 2. Does erotophobia-erotophilia moderate the relationship between the strongest predictor (anxiety, stress, or depression) and level of distress? 
$H_{0}$ 2: Level of erotophobia-erotophilia will not moderate the relationship between the most significant predictor and level of distress in women with PGAD.

$H_{1}$ 2: Level of erotophobia-erotophilia will moderate the relationship between the most significant predictor and level of distress in women with PGAD.

The null and alternatives hypotheses for Research Question 3 were as follows:

Research Questions 3. Does the nature of perceived partner support moderate the relationship between the strongest predictor (anxiety, stress, or depression) and level of distress for PGAD women with partners?

$H_{0} 3$ : Nature of perceived partner support will not moderate the relationship between the most significant predictor and level of distress in women with PGAD.

$H_{1} 3$ : Nature of perceived partner support will moderate the relationship between the most significant predictor and level of distress in women with PGAD.

I conducted a moderation analysis using the PROCESS feature created by Hayes (2012) for use in SPSS to assess if depression, the most significant predictor of sexual distress, would be moderated by erotophobia-erotophilia or by the nature of perceived partner support. Fifty-one participants were again analyzed for the erotophobiaerotophilia moderation and 38 participants (those who indicated they had partners and completed the Berlin Social Support - Actually Received Support scale (BSSS)) were analyzed for the perceived partner support moderation.

The moderation analysis revealed no statistically significant alteration in the variance accounted for when the interaction effect between the Sexual Opinion Survey scores and depression scores was computed $(b=-0.001,95 \%$ CI [-0.034, 0.032], $t=$ 
$-0.45, p=.965)$. The relationship between depression and sexual distress measured by the FSDS-R is not moderated by erotophobia-erotophilia (see Table 8). Similarly, no statistically significant alteration in the variance was accounted for when the interaction effect was computed between the nature of perceived partner support and depression scores moderation analysis $(b=-0.005$, 95\% CI [-0.079, 0.069], $t=-0.15, p=.883)$. The relationship between depression and sexual distress measured by the FSDS-R is not moderated by perceived partner support (see Table 9). I failed to reject the null hypotheses for research questions 2 and 3.

Table 8

Moderation Analysis of Erotophobia-Erotophilia

\begin{tabular}{|c|c|c|c|c|c|c|}
\hline & $b$ & se & $t$ & $P$ & $L L C I^{2}$ & $U L C I^{3}$ \\
\hline constant & 38.224 & 1.90 & 20.14 & 0.00 & 34.42 & 42.05 \\
\hline SOS $^{1}$ total score & 0.005 & 0.10 & 0.05 & 0.96 & -0.20 & 0.21 \\
\hline Depression total score & 1.263 & 0.33 & 3.81 & 0.00 & 0.60 & 1.93 \\
\hline Interaction effect & -0.007 & .02 & -0.45 & 0.96 & -0.03 & 0.03 \\
\hline
\end{tabular}


Table 9

Moderation Analysis of Partner Support

\begin{tabular}{|c|c|c|c|c|c|c|}
\hline & $b$ & se & $t$ & $p$ & $L L C I^{2}$ & $\mathrm{ULCI}^{3}$ \\
\hline constant & 38.736 & 2.20 & 17.57 & 0.00 & 34.26 & 73.22 \\
\hline BSSS Total Score $^{1}$ & -0.184 & 0.21 & -0.88 & 0.39 & -0.61 & 0.24 \\
\hline Depression Total Score & 1.307 & 0.41 & 3.21 & 0.00 & 0.48 & 2.13 \\
\hline Int_1 & -0.005 & .04 & -0.15 & 0.88 & -0.08 & 0.69 \\
\hline
\end{tabular}

\section{Women with Partners vs. Women without Partners}

\section{Hypothesis - Research Question 4}

The null and alternative hypotheses for Research Question 4 were as follows:

Research Questions 4. Is there a difference in distress levels for PGAD women with partners compared to PGAD women without partners?

$H_{0} 4$ : Level of distress will not be different for PGAD women without partners than for PGAD women with partners.

$H_{1} 4$ : Level of distress will be different for PGAD women without partners than for PGAD women with partners.

I conducted a one-way ANOVA analysis to examine differences in sexual distress scores by relationship status. Fifty-one participants were again analyzed, with 38 women completing the BSSS scale (those who indicated they had partners and completed the 
Berlin Social Support - Actually Received Support scale). The independent variable (partnered) included two levels: women with partners and women without partners. The dependent variable was the FSDS-R score (sexual distress). The results indicated no statistically significant difference in FSDS-R scores between women with partners (n = 38) and women without partners $(\mathrm{n}=13), F(1,49)=.23, p=.630$. Mean FDSD-R scores for partnered versus not partnered are shown in Table 10. Therefore, I failed to reject the null hypothesis.

Table 10

Partner Descriptives

\begin{tabular}{lllll}
\hline Partner status & $M$ & $S D$ & $S E$ & $C I$ \\
\hline Partnered & 23.54 & 13.72 & 4.06 & {$[16.24,30.61]$} \\
Not partnered & 25.82 & 14.93 & 2.38 & {$[21.22,31.02]$} \\
\hline
\end{tabular}

\section{Further Analyses}

Because several participants reported SSRI or other anti-depressant use, I used a Mann-Whitney $U$ test (Table 11) on current, past and both current and past antidepressant use with sexual distress measured by the FSDS-R as the dependent variable. This test determined that there is no difference in women who are currently using one or more of these medications when compared to women who used these medications in the past, or when compared to women who are both currently using these medications and used one or more in the past. 
Table 11

Sexual Distress in SSRI and Other Antidepressant Use Comparison to Non-use

\begin{tabular}{lccccccc}
\hline & & & & \multicolumn{3}{c}{ Standard } \\
& Mann- & Wilcoxon & Test & \multicolumn{3}{c}{ Test } & \\
& Whitney $U$ & $W$ & Statistic & SE & Statistic & $p$ \\
& & & & & & & .601 \\
Use of medication & & & & & & \\
Current/Noncurrent & 270.50 & 441.50 & 270.50 & 50.683 & -.523 & \\
Past/Non-past & 193.00 & 1013.00 & 193.00 & 43.621 & -.619 & .536 \\
Both & 326.00 & 579.00 & 326.00 & 52.527 & .133 & .894 \\
\hline
\end{tabular}

\section{Comments from Open-Ended Questions}

This is not an exhaustive review of the qualitative data, but selected as illustrative of the aforementioned points.

\section{First Open-Ended Question}

The first open-ended question sought to determine if participants felt there was a relationship between the onset of their PGAD and any specific trigger: "If participant answered “yes” to association of PGAD with a specific event, please specify event.” Of the 51 respondents, 31 provided comments indicating potential triggers, the majority of which pertained to medication use or withdrawal. One respondent indicated that “stopping SSRI trials (Prozac, Effexor, and Brintellix)” may have triggered the onset of her PGAD. Another respondent indicated a trigger of "switching my "cocktail" of antidepressants around". A third respondent indicated "I'm not sure if it was stopping the Prozac or a fall but they happened at the same time”; while a fourth similarly indicated 
“the abrupt discontinuation of SSRI”. Other responses included "birth control pills. Also Pn nerve injury from Zumba” and “pregnancy” as possible triggers.

\section{Second open-ended question}

The second open-ended question sought information on how the respondent's life was affected by PGAD: “Please describe what ways, if any, PGAD has affected your life.”

Of the 51 respondents, all of them supplied comments to this question. One respondent describes how she "had to quit my career job early. I became dependent on pain meds. I can't make solid appointments. I feel like an invalid much of the time. PGAD intrudes at the worst times.” Another respondent mentions how "over the years it has debilitated me. Cost me to lose my marriage. Has caused me to be unable to work. Depressed me.” Another respondent has “dealt with suicidal thoughts, anxiety that lasts for days, and I have become very anti-social. PGAD dictates what I can and cannot do because my life is scheduled around my times of masturbation, which is every 7 to 10 days. I have depression and suicidal ideation because of PGAD.” Another respondent discusses how "pgad has destroyed my life. I am unable to leave my house. Unable to sit, unable to wear anything but very loose clothes, and am thoroughly miserable. I miss intimacy.” Similary, another respondent indicates “It has ruined my life...causes me divorce.... My husband made me and my family think I was crazy and I was worthless....”. 


\section{Third open-ended question}

The third open-ended question sought to hone in on which aspect of PGAD was having the most impact on the quality of life of the respondent: "What aspects of PGAD, if any, do you feel have most impacted your quality of life?” This is important to consider for therapeutic intervention of symptom management and increasing quality of life for these patients.

Of the 51 respondents, all of them provided comments to this question. One respondent wrote that "constantly being aroused never knowing if I can go outside or not. Mentally making me feel like I'm a freak. Cost me my family” impacted her quality of life. Another respondent indicated "the pain, the embarrassment involved in seeking help, and the almost-impossibility of discussing it with anyone, even close friends. it's a very lonely condition.” Another telling comment from one of the respondents was "I had to quit work which had affected our income. We had to move our house we owned to a senior mobile home park. I didn't try to get Disability until just recently and then they told me it was too late because I was already getting my Social Security. Sometimes I just don't feel like I want to be with people because I feel too uncomfortable and I used to be such a people person! The biggest thing I think that bothers me is that my brain is so off! It's just too difficult to concentrate! I miss so much or I forget and I'm sure this ongoing arousal has a lot to do with it!” Another comment made was “I feel like I am useless and do not understand why I didn't just die in the OR" and similarly "Isolate myself at times don't want to go on.” 
Finally, one of the longest comments by a respondent indicated "PGAD has altered my entire life. I have not been able to have a healthy sex life with my husband of 21 years, I am limited as to being the kind of mother I'd like to be for my son, for example, I'd love to go see movies with him, spend more time with him, even at home, because I want to be supportive and involved in his life, but since his birth, my PGAD has limited our relationship, because it turns my mind inward, and I retreat to my own, quiet space where nothing will disturb my fragile state of mind. Before PGAD I was out going, able to socialize, spend time with family, focus on other people and travel. All of this has been taken away by PGAD. Now that other health issues are arising, such as I found out yesterday that I have osteoarthritis, I honestly fear the future. My father killed himself in 2007 in my front yard due to chronic pain, and I fear every day that that is how my life will end, and I fear the pain I know it will inflict upon my family.”

\section{Summary}

In chapter 4, I provided an outline of the results of the four research questions under study, as well as several frequencies and descriptive analyses. Analysis of the survey data indicated anxiety was not the strongest predictor of sexual distress in women with PGAD. Utilizing the FSDS-R (Female Sexual Distress Scale - Revised) as a measure of sexual distress, I concluded the strongest predictor of the three predictors under study using the DASS-21 (Depression Anxiety and Stress Scale - 21 items) turned out to be depression. . Neither erotophobia/erotophilia nor the nature of partner support moderated the effect of depression on sexual distress measured by the FSDS-R. There was also no significant difference between the group of participants with partners and the 
group of participants without partners. Samples were provided of comments received for the three open-ended questions asked in the survey. 
Chapter 5: Discussion, Conclusions, and Recommendations

\section{Introduction}

The purpose of the study was to examine predictors (anxiety, depression, stress) and moderators (erotophobia/erotophilia and partner support) of sexual distress in women with PGAD. I measured these variables using the DASS-21, the FSDS-R, the SOS, and the BSSS.

In the study, I examined (a) whether or not anxiety would be the strongest predictor, (b) whether or not erotophobia-erotophilia moderated the relationship between the strongest predictor and the level of sexual distress, (c) whether or not the nature of perceived partner support moderated the relationship between the strongest predictor and the level of sexual distress measured by the FSDS-R, and (d) whether or not there is a difference in sexual distress levels for PGAD women with partners compared to PGAD women without partners. In this chapter, I provide a detailed discussion and interpretation of the results presented in Chapter 4. Limitations of the study, recommendations for future studies, and implications for social changed will also be provided.

\section{The Significant Predictors}

\section{Correlations}

I found statistically significant correlations among depression, anxiety, and stress. Also, age was significantly correlated with depression and stress. Interestingly, differences in mean scores on each of the DASS-21 subscales (anxiety, depression, stress) as well as the FSDS-R were quite noticeable when comparing scores with 
employment status, with those being non-employed having the highest mean scores. Still, cause and effect conclusions cannot be drawn given that the study was a crosssectional single-point in time, and not longitudinal in nature. It can be hypothesized, however, that the distress experienced from PGAD symptomatology make it difficult for women with PGAD to hold a job. The comments provided to the open-ended questions further strengthen this hypothesis.

While there was no evidence to suggest any one of the underlying conditions presented in the survey (Tarlov cysts, restless leg syndrome, overactive bladder, pudendal neuropathy, and sexually transmitted infections) was significantly correlated with sexual distress, many of the participants are facing multiple medical diagnoses from the aforementioned which did correlate with sexual distress. Interestingly, multiple medical diagnoses were not correlated to depression, possibly due to the large antidepressant usage reported by participants. Number of pregnancies was also significantly correlated with sexual distress. It could be hypothesized that this correlation was due in part to respondents seeking relief of symptoms and ultimately becoming pregnant. It could also be hypothesized that the number of pregnancies contributed to the onset of the symptoms of PGAD indirectly due to subsequent pregnancy-related etiologies such as pelvic floor concerns, bladder concerns, or nerve related issues. Bilgic and Kizilkaya Beji (2014) discussed the potential role risk factors such as pregnancy and pelvic floor muscle atrophy on sexual dysfunction and quality of life for women with lower urinary tract symptoms. Both number of medical diagnoses and number of pregnancies should be examined in more depth in future research to explore cause and effect. 
No correlations were found between sexuality, religion, spirituality, number of births, erotophobia-erotophilia, or partner support with sexual distress. It is possible that correlations were lacking with respect to sexuality and erotophobia/erotophilia due to lack of diversity in the responses to these questions. The majority of respondents were heterosexual or predominantly heterosexual, and levels of erotophobia were low. Most of the respondents had average scores on the erotophobia/erotophilia scale, falling somewhere in-between, or in the neutral range. Similarly, a wide range of diversity did not present itself in the area of religion. While more diversity was present in the area of spirituality, it could simply be that sexual distress resulting from symptoms of PGAD does not discriminate based on spirituality, religion, or sexuality. Further discussion on erotophobia-erotophilia and partner support appear later in the chapter.

Duration of PGAD was also not correlated to sexual distress nor to age, depression, anxiety, or stress; however, age was significantly correlated to depression and stress. Theoretically, it could be that sexual distress does not change over the duration of this particular disorder considering the nature PGAD symptomatology. It could also be theorized that, given the cross-sectional nature of the study, it could simply be a matter of the current level of sexual distress at the time of the survey by those who were willing to participate. Longitudinal studies may show changes in distress levels over the course of time, such as that in Cooley, Short, and Moriarty’s (2003) study of distress in patients with lung cancer. 


\section{Depression Versus Anxiety and Stress as Predictors}

Given findings from prior studies suggesting the possibility that symptoms are due to high sensitivity towards one’s self and one’s surroundings (Leiblum \& Chivers, 2007; Leiblum, Seehuus, Goldmeier, \& Brown, 2007), I hypothesized that anxiety would be the most significant predictor of the three psychological factors under study. I further based this hypothesis on the model proposed by Leiblum and Chivers (2007) whereby it was suggested that women’s appraisal, response and potential hypersensitivity to detection and awareness of genital sensations may cause them to experience anxiety, triggering the sympathetic nervous system to arouse and enhance their not only their perception and awareness of the genital sensations but also their attentiveness to such sensations. Interestingly, although anxiety had a statistically significant correlation to sexual distress, when competing with depression as a predictor, it did not add to the prediction, nor did stress. Instead, depression severity surfaced as the best statistically significant predictor of the three psychological factors under study. Although several prior studies have noted high rates of depression in PGAD participants as assessed by self-report (Pink, Rancourt \& Gordon , 2014; Leiblum et al., 2005), only one prior study has assessed depression, anxiety, or stress with the use of a validated measure like the DASS. Validated measures provide more thorough constructs of the factors being measured. Garvey et al. (2009) noted the use of the Hospital Anxiety and Depression Scale in a study assessing spontaneous genital arousal and PGAD of clients of a walk-in sexual health clinic in London; however only one participant in their study of 96 participants indicated having all five criteria of PGAD. 


\section{Theories Behind the Results}

There are several relevant theories that bear on my interpretation of the results; however, this exercise is made extremely challenging due to the overlap between the certain aspects of the underlying constructs of depression, anxiety, and stress.

To begin with, although depression is prevalent in anxious individuals and vice versa (Tiller, 2013), the construct of depression is clearly distinct from the construct of anxiety. Early research by Lazarus (1991) showed that anxiety is constructed as a response to perceived threat, whereas depression is a response to perceived loss (i.e. loss of self-worth, interest, person(s), job, tangible assets).

Anxiety has historically been understood within two divergent constructs: (a) as an acute emotion (state anxiety), or (b) as a personality construct (trait anxiety). State anxiety is generally defined as an unpleasant emotional arousal in the face of a threat or danger stimulus, with a cognitive appraisal of a threat as a prerequisite for the experience of this emotion (Lazarus, 1991). Conversely, trait anxiety is thought to reflect the existence of stable individual tendency to respond with state anxiety in the anticipation of threatening situations.

Further complicating the picture, at the level of both state and trait anxiety, is a delineation that has been proposed between worry and emotionality (Spielberger, 1980), with worry referring to the cognitive component of the anxiety experience, and emotionality referring to the perceived arousal component of the anxiety experience. Although these two components are usually present at the same time to some degree, they are only poorly- to moderately-related to each other. Importantly, worry is negatively 
correlated with perceived self-efficacy (Schwarzer, 1996). Thus, people who do not feel able to cope with challenging situations may experience self-doubts and worry, thus becoming more anxious.

Last, a stress response may include an anxiety component, but not always. As researchers such as Endler (1997) and Keane, Taylor, and Penk (1997) noted, stress may implicitly be thought of in the following means: (a) as a stimulus or stressor; (b) as a response to such a stressor which would be deemed as symptoms experienced; (c) or as a situational encounter experienced by an individual.

\section{Understanding the Current Findings}

First, depression surfacing as the strongest predictor over anxiety and stress is interesting. Depression may be present as a response to loss, and it is easy to understand how these women who have experienced loss of normalcy could develop such symptoms, and that these would indeed predict distress. Prior PGAD research does point to depression as a prominent psychological factor in PGAD cases. For example, Eibye and Morch Jensen (2014) discussed the case of a patient hospitalized in a psychiatric unit and diagnosed with agitated depression upon the onset of PGAD. Notably, the onset of PGAD occurred after the cessation of paroxetine, an antidepressant she had been taking for a history of anxiety and depression. Leiblum et al. (2007) noted early on in their PGAD research studies that depression was more likely to be present in women that met all criteria for PGAD than in women who did not (55\% compared to 38\%), and that they were also more likely to be anxious. In some cases depression may occur before the onset of PGAD and in other cases after the onset of PGAD. Yet, in other cases the 
temporal relationships remains unclear. Undoubtedly, it is still valid to hypothesize that the loss of normalcy experienced by women contributes to the development or worsening of depressive symptoms and sexual distress.

Depression has also been highly associated with chronic pain disorders (Knaster, Estlander, Karlsson, Kaprio \& Kalso, 2016). Extensive volumes of literature can be found examining the comorbidity of chronic pain and depression (Outcalt et al., 2015). Pink et al. (2014) noted that over half of the 15 participants in their PGAD study indicated pain as a symptom of their PGAD. Given the consideration that PGAD is not a disorder of sexual dysfunction, it could be suggested that PGAD potentially be categorized as a pain disorder; perhaps a subcategory of pelvic pain.

Turning to anxiety, it is reasonable to consider that both state and trait anxiety may be present in women with PGAD; however, to properly test this hypothesis would require a more precise assessment of anxiety using a measure such as the State-Trait Anxiety Inventory [STAI] (Spielberger, 1983). This is a self-report inventory consisting of 20 items assessing state anxiety and another 20 items assessing trait anxiety.

If state anxiety is considered as a specific response to an event or stimulus considered as threatening, then it is easy to see how cognitive recognition of unwanted intrusive genital sensations would cause women who experience PGAD symptoms to experience state anxiety. It is possible that coping mechanisms are a factor; although this was not examined in this study so can only be theorized as an explanation for why anxiety, while being present, is not a predictor of sexual distress in this study, and instead may be a contributor, along with stress, to depressive symptoms that appear to be 
prevalent in the majority of the study participants both prior to the onset of and after initiation of PGAD symptoms. This, however, does not explain the women who indicated they have had PGAD since early childhood or prior to any experience of depression or the intake of antidepressant medication. In these women, it may be that trait anxiety is present. It is plausible that some women with trait anxiety, when experiencing unintended or unwanted genital stimulation, process it against a propensity for anticipation of such a stimulus. Thus the possibility exist that state anxiety, trait anxiety, or both may be present in women experiencing PGAD. It is difficult to compare anxiety in this study to prior PGAD studies since only one used a validated anxiety instrument and only one patient in that study presented with PGAD. Further studies are needed that examine anxiety in more depth in women with PGAD; that delve into the intricacies of the physiological and psychological effects of anxiety and how or if these effects may or may not be contributing to PGAD. Gathering historical information on respondents’ anxiety along with current information and measuring anxiety levels over time during the course of their PGAD can add to the significant gap in the current literature.

Turning to stress, it can be considered as one of the following: (a) as a stimulus or stressor; (b) as a response to such a stressor which would be deemed as symptoms experienced; (c) or as a situational encounter experienced by an individual. Against this background, one can understand women with PGAD experiencing significant stress contributing to anxiety following PGAD symptoms as a critical event or as a part of the response pattern in anticipation of same, yet not predicting sexual distress per se. 
Last, another explanation for anxiety or stress not being significant predictors of sexual distress could be individualized allostatic responses. Allostasis is defined as "the process of achieving and maintaining stability in the internal environment” (KendallTackett, 2010, p. 184). Research suggests that gender-specific coping mechanisms play a role in perceived stress, allostatic load (stressors impeding allostasis), and responses. This contributes to stress-induced neurobehavioral disorders such as anxiety and depression. With that said, it can be hypothesized that stress, while not being a statistically significant predictor of sexual distress, can indirectly contribute to such distress by directly contributing to depression, a significant predictor among PGAD sufferers in this sample under study.

Another factor to consider, as mentioned previously, is the instrument I used in this study, which may not have been extensive enough to capture significant anxiety and stress levels considering each psychological factor consisted of only 7 questions each. Since this is the first PGAD study to use a validated measure of anxiety, depression and stress, aside from the one study previously discussed that used the Hospital Anxiety and Depression Scale whereby only one patient presented with PGAD, it is difficult to determine the strength of the instrument used. Further research should seek to use both this instrument again as well as more extensive measurements of psychological factors.

\section{Signals in the Medical Backgrounds and Comments}

The results are less puzzling upon consideration of two further items: the correlation of multiple medical diagnoses to FSDS-R distress levels faced by many of the participants, and the examination of the comments provided by the participants in the two 
open-ended questions assessing the impact of PGAD on their lives. It could be hypothesized that the combination of multiple medical diagnoses could be a sign of an overall syndrome that has been manifesting itself through multiple outlets. This would need considerable resources to expand the research in future studies such as physical examinations, laboratory tests, etc. It could also be hypothesized that the combination of multiple medical diagnoses with PGAD could potentially contribute to one's depression, even though the correlation in this dissertation did not exist. The lack of correlation in this study could be due to the high rate of anti-depressant use by participants. Also, I did not study coping mechanisms for psychological factors (depression, anxiety, and stress) in this dissertation.

In a study of 7,620 Australian participants from primary care facilities, Gunn and associates (2012) noted that rates of depression increased as comorbidity of chronic illnesses increased. This begs the question as to whether other underlying medical diagnoses could be an etiological factor among PGAD patients that have yet to be presented in current literature, such as autoimmune disorders or other chronic illnesses.

The comments provided by the participants in the open-ended questions of the survey offer many evocative reflections and considerations as to possible explanations of why depression was found to be the most significant predictor. For example, the comments included remarks regarding: low quality of life; life is ruined; depression; quit working; crippling; worthless; ruined sex life; miserable; want to die; withdrawn; distress, distracting; difficult to concentrate; hopelessness. Many of the respondents could no longer hold jobs or could only work part-time. Similar quality of life concerns 
can be found among other chronic illnesses such as fibromyalgia. Bernard, Prince and Edsall (2000) noted comparable conclusions with respect to quality of life in fibromyalgia patients, including poor mental health, reduced functionality in career management and negative impact on personal relationships. Whether these items contributed to anxiety, stress and depression levels or vice versa are difficult to assess in a cross-sectional study. Longitudinal studies would provide better opportunities for evaluation of which may occur first.

\section{Possible Connection to SSRIs/SNRIs}

Comment after comment suggests the poor quality of life for women with PGAD. Many have been on antidepressants either in the past or are currently using these medications. In fact, antidepressant use, including SSRIs/SNRIs, was quite high in this study. Over three-quarters of the participants have used one or more of these medications in the past, while nearly 50\% are using them currently. Medications reportedly used include: citalopram, escitalopram, fluoxetine, fluvoxamine, paroxetine, sertraline, duloxetine, venlafaxine, milnacipran, bupropion, mirtazapine, nefazodone, trazodone, vilazodone, and vortioxetine.

While cause and effect cannot be assessed here regarding the relationship between depression and PGAD, it is of note that 16 of the 51 participants reported the onset of their PGAD in close temporal proximity to either starting, changing, or withdrawing from antidepressant therapy, which suggests that depressive symptomatology preceded the PGAD and not the reverse. This is not to suggest that depression causes PGAD; but rather to consider the large number of respondents who indicated SSRI use and/or 
withdrawal prior to onset of PGAD symptoms. It is reasonable to suggest further research into the effects of SSRI use and withdrawal and why these drugs could potentially cause symptoms such as those related to PGAD. Also, while there is face validity to the hypothesis that PGAD could also cause depressive symptoms, this is clearly a line of enquiry worth further exploration. Future studies should examine the onset of depression compared to the onset of PGAD; the start of use and withdrawal of SSRIs compared to the onset of PGAD; and depression levels over time in women with PGAD.

Since so many respondents reported PGAD symptoms in close proximity to SSRI use, it is important to examine the effects of an SSRI on the human brain. Increases of serotonin (5-HT) levels act on several areas of the body such as the brain, the spinal cord and the GI tract. Only 1-2\% of serotonin resides in the brain (Lichtblau, 2011). There are several 5-HT receptors - seven classes and fourteen known receptors (Nestler, Hyman, \& Malenka, 2009). Different 5-HT receptors act on different areas. Of these 5HT receptors, 5-HT1A and 5-HT2A receptors are found to be the key intermediaries of serotonergic stimulation of the hypothalamic-pituitary-adrenal axis of the brain (Hanley \& Van de Kar, 2003). The hypothalamus, located in the diencephalon portion of the brain, is considered to be the area of the brain responsible for satiety. An action on the 5HT2A receptor can have an effect on spinal reflexes related to orgasm and ejaculation, which can ultimately contribute to sexual dysfunction (Lichtblau, 2011). Neuroscientist Jim Pfaus has conducted extensive research on pathways of sexual desire. He thoroughly examined the relationship between excitation, inhibition, neurochemical and 
neuroanatomical systems and how neurotransmitters such as serotonin play a role in these and satiety. Pfaus (2009) noted that serotonin neurons arising from the midbrain propel axonal projections to several sites throughout the brain, including the hypothalamus, as well as to the spinal cord and lower lumbar-sacral regions controlling genital reflexes. It is reasonable then, considering the work of Pfaus and the findings of the current study to hypothesize that sudden stoppage of a serotonin reuptake inhibitor, causing a sudden drop in 5-HT, may be causing a "short-circuit" of the neurons of the hypothalamus, brainstem and spinal reflexes associated with sexual arousal and orgasm, reducing the ability to recognize satiety of sexual response and arousal thereby allowing arousal to reach extreme heights. Hence, the high rate of respondents indicating that PGAD began upon sudden withdrawal of SSRIs.

A new study by King, Goldstein and Pfaus (2016) found that treatment given for sleep (zolpidem, also known as Ambien) in a PGAD patient subsequently provided significant, though temporary, relief of PGAD symptoms. Zolpidem is a fast-acting sedative-hypnotic, indirect GABA A receptor agonist with a short half-life of 2-3 hours that initiates a GABA release while inhibiting dopamine release, thereby releasing serotonin. This study further supports the aforementioned hypothesis that serotonin may be playing a significant role in the brain circuitry of patients with PGAD.

With depression appearing to be so prevalent among PGAD sufferers, it is important for the healthcare community to consider treatment options that not only target PGAD physical symptoms, but also the psychological symptoms that coincide. Given the history of many of these patients with pharmacological therapies, antidepressants may not 
be the best or only treatment solution for targeting depression in these patients.

Clinicians may want to also consider treatment plans that include such strategies as cognitive behavioral therapy or other forms of non-pharmacological treatments.

\section{The FSDS-R}

In hindsight, a significant oversight in the early work of Lieblum and others was the failure to delve into the experience of distress in a systematic, in-depth manner. In her seminal papers first characterizing the disorder, the presence of distress using the criteria of "the persistent feelings of genital arousal feel intrusive and unwanted" was apparently captured using a single item rating distress on a scale of 1-10, whereby 1-3 was low, 4-7 was moderate and 8-10 was high. A valid measure of sexually-related distress was never utilized. Despite this, in 2007 the disorder was re-conceptualized from persistent sexual arousal syndrome to a non-sexual disorder by Leiblum et al. (2007), leading to a change in nomenclature from sexual to genital arousal disorder as the arousal was noted as genital in nature but not triggered by sexual stimuli or thoughts. Of note, the nomenclature change was not based on evidence that sexuality was not affected or distressing but rather on the basis of the observation that the symptoms of arousal were present in the absence of sexual stimuli. Prior studies, however, did not use a valid measure of distress such as the FSDS-R to measure sexual distress in this population. Although PGAD may not necessarily be a disorder of sexual arousal, the severity of sexual distress that it can cause in women with PGAD may be overlooked by clinicians as a result of the de-emphasis on sexual arousal. 
My study extends the information previously available and provides new evidence for further hypothesis generation by being the first to use the FSDS-R in a study of PGAD. A score of greater than 11on the FSDS-R is considered the cutoff point for sexual distress discriminative of sexual dysfunction (DeRogatis et. al, 2008). The high levels of sexually-related distress as assessed by the FSDS-R (mean 25.24) in my study demonstrates that women experience significant sexual distress, even if the origin of that distress is not based on sexual arousal in response to sexual cues / desire per se. When compared to FSDS-R mean scores from studies of other categories of FSD, it is notable that the mean score from my study is as high or higher than found in the majority of studies where the instrument has been used. For example, use of the FSDS-R in samples of women with hypoactive sexual desire disorder (HSDD) in a study by DeRogatis et al. (2011), reported mean FSDS-R scores were 24.73; while in a pilot study by Runels, Melnick, Debourbon and Roy (2014) for the treatment of women with painful intercourse and/or anorgasmia, the mean FSDS-R score prior to any treatment was 17.27. Thus, the distress expressed by participants with PGAD in my study can be understood in to be in the more severe range of sexually-related distress. It is possible that this severity reflects selection bias in that selection of women who have sought treatment and/or who have enough distress to join a support group may not reflect the severity in the overall population of women with PGAD. Nevertheless, in the absence of other studies using valid measures of sexually-related distress, it is important to assume that such distress levels are likely. This consideration of severity is also warranted considering the FSDS$\mathrm{R}$ was not created specifically for or validated in PGAD patients, and the high scores on 
non-arousal related items suggest the possibility that the sexual distress that affects the lives of these women is far greater than simply the effect on genital arousal.

My findings also suggest the possibility that re-conceptualizing PGAD for the last decade in a manner that deemphasizes the sexual impact of the disorder may have been premature and indeed, may have contributed to increasing the challenges women with the condition may experience in obtaining appropriate care. As an example, while no one would consider spinal cord transection a sexual disorder, it is nevertheless well recognized that people with such a lesion may experience profound sexual dysfunction and indeed, care of spinal cord-injured patients is now considered incomplete without attention to the sexuality of the subject. By illustrating the profound levels of sexual distress women with PGAD experience, my findings highlight the importance of providing holistic care for the sexual concerns these women experience, beyond focusing on the genital symptoms alone.

Also important, as evidenced by the sample of comments displayed in chapter 4 that participants provided on the three open-ended questions, the development of a PGAD-specific distress scale would be a valuable contribution to the study of PGAD. Comments provided revealed a significant negative impact on quality of life for women with PGAD beyond the sexual distress noted, ranging from inability to focus in all aspects of life and a profound effect on their ability to work, to the extreme extent of suicidal ideation; though it should be noted that poor quality of life could be influenced by other factors not necessarily related to PGAD. For the average person, it would be similar to an itch that does not get relieved by scratching just once, such as in the case of 
poison ivy; or similar to having a continuous eye twitch that won’t subside. It is uncomfortable; not necessarily painful, but rather a constant feeling of discomfort which can be quite severe in some cases. The comments provided by the participants reveal a multitude of reasons for the distress experienced.

Comments provided by participants insinuate that loss of control over one's life is a large contributor to distress. As Wallston, Strudler Wallston, Smith, and Dobbins (1987) explain, loss of perceived control over internal states and behavior can have a significant impact on health and health outcomes. By utilizing qualitative methodologies, measures could be generated that cover aspects regarding perceived control as well as other potential contributors to distress in people with PGAD and could include such questions as : on a scale of $\mathrm{x}$ to $\mathrm{y}$, how much control do you feel you have over your life; are you distressed by feelings of physical pain and if so how distressed; are you distressed by inability to focus and if so how distressed; are you distressed by the thought of making plans and if so how distressed; are you distressed about holding a job and if so how distressed; are you distressed about embarrassment and if so how distressed; are you distressed about talking to your partner about your symptoms and if so how distressed. This could potentially yield better discrimination from distressed versus non-distressed or non-PGAD samples. The development of such a PGAD-specific scale would advance research of PGAD given the current state of knowledge and our need to deepen our understanding of this condition. 


\section{The Moderators}

\section{Erotophobia-Erotophilia}

Another interesting and important finding in my study was the lack of moderation by erotophobia-erotophilia; nor was erotophobia-erotophilia correlated to or a significant predictor of sexual distress. In a recent study, Carvalho et al. (2015) noted sexual conservatism as a significant predictor of distress during intercourse for their sample of women with PGAD. These researchers, however, used the Sexual Dysfunctional Beliefs Questionnaire as opposed to the SOS, which could account for the difference in outcome. For the SOS, a mean score of 61 suggests that the respondents are in the neutral range of erotophobia-erotophilia. As noted in chapter 2, prior research found erotophobia to be present in several studies of sexual dysfunction (Furnham \& Hirandani, 2009; Fisher, Byrne, \& White, 1983; Byrne \& Kelley, 1986; Hall, Hogue, \& Guo, 2014; Macapagal \& Janssen, 2011; Wiegel, Scepkowski, \& Barlow, 2007). Sexual guilt, shame and fear of stigma were noted as relevant factors in many of these earlier studies (Cherner \& Reissing, 2013). Such was the case in a few of the comments provided by the participants of the present study (i.e. "it has made me feel wrong and dirty. It has made me question who I was, who I am and what caused it.”); however, the prevalence of these comments is low. Again, given the lack of erotophobia present in the study sample and the high incidence of erotophobia being empirically present in sexual dysfunction research, the case could be made that PGAD is not a disorder of sexually oriented dysfunction at all as previously noted. However, given the scores of the FSDS-R provided in this study, it is also quite clear that while PGAD may not be grounded under 
the realm of sexual dysfunction, it does appear to cause significant sexual distress coupled by a significant impact on quality of life. This is similar to other illnesses, such as cancer, that while not stemming from sexual dysfunction can cause significant impact on sexuality, sexual distress and quality of life. This leads to the idea that PGAD, while being associated with genital arousal, should be given consideration as a disorder that does not stem from a sexually-based etiology or negative affective responses to sexual stimuli as measure by the SOS, but rather some other biological pathological trigger that is intensified by and/or provokes psychological comorbidities such as depression and anxiety. Some clinicians are approaching PGAD from a non-sexual perspective. Dr. Robert Echenberg of the Echenberg Institute for Pelvic and Sexual Pain who suggests PGAD is a pain disorder as opposed to a disorder of sexual dysfunction (http://www.instituteforwomeninpain.com/dr.-echenberg-answers-questions-on-pgad, 2016).

The findings of my study suggest that while these particular respondents are suffering from PGAD, the average respondent is not erotophobic as evidenced by the scores of the SOS. This makes sense if PGAD is examined from the viewpoint of being a disorder without roots in sexual dysfunction, considering that erotophobia tends to be more prevalent in disorders of sexual dysfunction (Furnham \& Hirandani, 2009; Fisher, Byrne, \& White, 1983; Byrne \& Kelley, 1986; Hall, Hogue, \& Guo, 2014; Macapagal \& Janssen, 2011; Wiegel, Scepkowski, \& Barlow, 2007). This may also factor in to why anxiety failed to be a significant predictor of sexual distress in this study of women with PGAD. Studies suggest that negative emotions such as anxiety are related to factors 
such as sex-guilt and shame, significant characteristics found in erotophobia (Furnham \& Hiranandani, 2009). It can also be proposed that the finding may be a product of the small convenience sample used for the present study. Future studies should strive to capture larger samples.

\section{Partner Support}

Finally, the findings on partner support, while not found to be a moderating variable to sexual distress measured by the FSDS-R, nor statistically different sexual distress levels among the group with partners and the group without partners, are not inconsistent with literature describing a variety of individualized characteristics and perceptions on the part of the recipient of partner support. For example, while not all women in the study had partners (70.5\% with, $29.5 \%$ without), it is feasible to theorize that those who did may behold a variety of avoidance-type traits, including some with high attachment avoidance and some with low attachment avoidance (Girme, Overall, Simpson \& Fletcher, 2015; Dewitte, 2012). As discussed in chapter 2, high attachment avoidance traits are conducive to higher erotophobia levels (Peloquin, Bigras, Brassard \& Godbout, 2014). In my study, erotophobia levels were low to average. This would be conducive to my findings of no statistically significant moderation of partner support, particularly if the study participant sample has a low level of high attachment avoidance traits. Unfortunately, conclusions cannot be drawn regarding avoidance traits in this study.

It is quite possible that some participants of this study view particular features of partner support as adequate while others may view the same support as inadequate 
(Brock \& Lawrence, 2014). Unless these factors are examined through additional validated questionnaires and potentially involve surveying the partners as well, it is likely that fluctuations in research findings will continue to occur. Carvalho et al. (2015), while using the Dyadic Adjustment Scale Seven-Item Version as opposed to the Berlin Social Support - Actually Received Support scale, had similar findings of no statistically moderating significance of marital/relationship adjustment to severity of PGAD distress; they did find marital/relationship adjustment to be a significant predictor of PGAD distress when used as an independent variable as opposed to a moderator. While my study did not find partner support to be correlated to or a significant predictor of PGAD, it could again be theorized that individual personalities and perceptions of adequate partner support contribute to this finding.

For those without partners, it is possible that other means of support are available to this group such as family, friends, or the online support group. This group may use the healthcare setting as their means of support. Further inquiry into support systems would need to be conducted in future studies for those without partners.

Future studies observing partner support should consider adding in questionnaires that examine high versus low attachment traits as well as overprovision versus underprovision of support. Such measures will allow researchers to determine if the results of a scale such as the BSSS are in conjunction with the type of traits prevalent in the respondents of their studies. They should also consider including partners' responses in future study. This could lead to valuable insight as to how PGAD affects relationships and family quality of life. 


\section{Quality of Life}

Of importance to note is that while partner support did not moderate FSDS-R measured sexual distress in my study, other social factors that were not measured by scales do potentially contribute to the distress of several respondents. As the comments noted in chapter 4 reveal, PGAD sufferers are putting their lives on hold, quitting jobs, withdrawing from friends, family and life in general, avoiding situations where they need to leave their homes for long periods of time, and becoming isolated due to their symptoms. Some have remarked about embarrassment and shame. Some have suicidal ideation. Some have seen several clinicians with little relief, and some fear the clinicians are not hearing them or taking them seriously. This is not an exhaustive review of the qualitative data, but selected as illustrative of the aforementioned points.

Interestingly, when comparisons were made between the SDSM group and the online support group, depression scores were higher in the SDSM group. While several hypotheses can be made regarding these results (i.e. - this could suggest that the women from the SDSM group sought out treatment because they were more depressed than those in the online support group, or that the support received from other members of the online support group experiencing similar symptoms can provide a significant psychological benefit to PGAD sufferers) no generalizations regarding this finding can be substantiated until further research is conducted on quality of life concerns. Encouragement for PGAD sufferers to join support groups, however, may still be a consideration for clinician treatment plans. 


\section{Conclusions}

PGAD is a debilitating disorder; its sufferers endure a variety of physically, psychologically and socially distressing symptoms. Quality of life can potentially be severely impacted by this relatively unknown, seemingly rare disorder. The etiology of PGAD remains a mystery. Earlier studies of PGAD posited a variety of theoretical etiologies including biological, neurological, psychological, pharmacological and vascular. Anxiety, depression and stress have been targeted as potential triggers or exacerbating influencers of PGAD (Leiblum \& Chivers, 2007; Leiblum, Seehuus, Golmeier, \& Brown, 2007; Leiblum et al., 2005). Others studies have noted underlying factors such as conservative sexual beliefs to have a significant correlation with PGAD (Carvalho, Verissimo, \& Nobre, 2015). Literature on PGAD remains deficient. A handful of case reports have been presented as well as some studies with relatively small sample sizes. PGAD literature is lacking in all areas, including longitudinal studies and larger studies to further elucidate the many etiologies proposed.

My study has supplied evidence that PGAD is a paradoxical disorder in that while not triggered by sexual stimuli nor associated with negative affective responses to sexual stimuli as determined by the SOS scores, it is manifested in disordered genital response and is nevertheless experienced as profoundly distressing to the sexuality of women experiencing the condition. Clinicians need to be aware of the high levels of sexual distress and encourage open discussions with patients to better assist with the development of treatment plans. 
The finding that depression is a serious concomitant disorder in women with PGAD, whether the women are partnered or not, warrants continued assessment and close monitoring by clinicians caring for this population. Suicidal ideation is a recurring theme among this group and several suicides attributed to PGAD have occurred within the online support group (but not within the study sample) during August of 2016. The findings are indicative of the importance of utilizing the biopsychosocial framework model in the PGAD patient population given we still have no clear understanding of etiology or natural disease. Biological, psychological and social aspects should all be taken into consideration when forming treatment plans for these patients. Not only should healthcare clinicians seek to reduce or eliminate the physical symptoms (biological) of this disorder, but the psychological and social symptoms should be addressed as well. Quality of life scales should be a consideration when designing future studies of PGAD. These women, both partnered and un-partnered, are not only suffering from the biological symptoms PGAD, they are also suffering from psychological symptoms such as depression and social symptoms such as the inability to live a quality life (i.e. - no longer work, no longer make plans, no longer interact with friends and family). For those without partners, support groups can assist in providing psychological benefit by serving as an outlet for PGAD patients to share their stories, compare symptomatology, and reap the benefits from shared experience and understanding.

\section{Limitations of the Study}

Limitations of the study include the small sample size and correlational design methodology which limits interpretability of the relationships found. Power calculations 
indicated a sample of 64 would have been appropriate. After eliminating those participants that did not qualify from the original sample of 73 respondents, I was left with a smaller than desired sample of only 51 participants. As a result of the smaller sample size, the study may have been underpowered, perhaps contributing to the statistical significance of the results throughout the study. Secondly, participants were recruited from an online support group venue in addition to a clinician’s database. Several participants indicated they did not have an official diagnosis of PGAD and were self-reporting their disorder, which can contribute to bias being present in the study. Finally, a third limitation was the use of the FSDS-R, a validated scale examining female sexual distress as determination of distress in women with PGAD. This was due to the lack of a scale focusing on PGAD specific distress. No generalizations can be made from this study regarding cause and effect.

\section{Recommendations}

Recommendations from my study include performing larger, multinational studies in the future in order to generate data on the impact of culture on the disorder and to begin to better understand the epidemiology and natural history of the condition and provide more generalization to the population. Other recommendations include further studies of SSRI/antidepressant use in PGAD sufferers given the number of respondents who reported their use and the onset of PGAD upon cessation of use. It may be possible to query large pharma company datasets of SSRI adverse events to ascertain this phenomenon. Future studies should also consider the use of a valid suicidal ideation 
scale as well as utilizing psychological therapies for PGAD sufferers in hopes of reducing depression and suicide rates in conjunction with providing coping strategies.

Given that the majority of the variance in sexual distress in the sample cannot be accounted for, future work should strive to better understand the nature of the distress these women experience beyond the realm of sexual distress. I also suggest using more extensive psychological measurements for anxiety, depression and stress in future studies, including measurements examining perceived control. Considering the multiple medical diagnoses reported by participants, future studies may want to consider other underlying medical conditions that may be found to be common among PGAD patients such as autoimmune disorders for example.

\section{Implications for Social Change}

Implications for social change are significant. Women with PGAD have a high rate of depression. Spotting this depression early on while working through other PGAD treatment strategies can encourage early treatment and control of depression, and subsequently lower suicide rates from this terrible disorder. The comments collected in my study from the participants are indicative of a group of women who are pleading for help from our healthcare system. They want to be heard. They want answers. They want to be taken seriously. They want their suffering to end. They want their lives back. For this to occur, more PGAD research needs to be done. Healthcare clinicians need to be educated on this disorder and they need to take their patients complaints and cries for help seriously. This study needs to be presented to clinicians around the globe who can work to help treat the depressive symptoms of PGAD while searching for other 
underlying pathology. Publications from my study can form a positive contribution to the education of healthcare clinicians worldwide. 


\section{References}

Adegunloye, O. A. \& Gwabachi Ezeoke, G. (2011). Sexual dysfunction - A silent hurt: Issues on treatment awareness. Journal of Sexual Medicine, 8, 1322-1329. doi:10.1111/j.1743-6109.2010.02090.x

Althof, S. E., Leiblum, S. R., Chevret-Measson, M., Hartmann, U., Levine, S. B., McCabe, M., ... Wylie, K. (2005). Psychological and interpersonal dimensions of sexual function and dysfunction. Journal of Sexual Medicine, 2(6), 793-800. doi:10.1111/j.1743-6109.2005.00145.x

American Psychiatric Association. (2013). Diagnostic and statistical manual of mental disorders (5th ed.). Arlington, VA: American Psychiatric Publishing.

Amirkhan, J. H., Urizar Jr., G. G., \& Clark, S. (2015). Criterion validation of a stress measure: The stress overload scale. Psychological Assessment, 27(3), 985-996. doi:10.1037/pas0000081

Anzellotti, F., Franciotti, R., Bonanni, L., Tamburro, G., Perrucci, G., Thomas, A., ... Onofrj, M. (2010). Persistent genital arousal disorder associated with functional hyperconnectivity of an epileptic focus. Neuroscience, 167, 88-96. doi:10.1016 /j.neuroscience.2010.01.050

Barlow, D. H. (1986). Causes of sexual dysfunction: The role of anxiety and cognitive interference. Journal of Consulting and Clinical Psychology, 54(2), 140. doi:10.1037/0022-006X.54.2.140

Barth, K. R., Cook, R. L., Downs, J. S., Switzer, G. E., \& Fischoff, B. (2002). Social stigma and negative consequences: Factors that influence college students’ 
decisions to seek testing for sexually transmitted infections. Journal of American College Health, 50, 153-158. doi:10.1080/07448480209596021

Basson, R., Althof, S., Davis, S., Fugl-Meyer, K., Goldstein, I., Leiblum, S., ... Wagner, G. (2004). Summary of the recommendations on sexual dysfunctions in women. Journal of Sexual Medicine, 1(1), 24-34. doi:10.1111/j.17436109.2009 $.01617 . \mathrm{x}$

Battaglia, C. \& Venturoli, S. (2009). Persistent genital arousal disorder and trazodone, morphometric and vascular modifications of the clitoris. A case report. Journal of Sexual Medicine, 6, 2896-2900. doi:10.1111/j.1743-6109.2009.01418.x

Bedell, S., Goldstein, A. T., \& Burrows, L. (2014). A preclitoral mass as a cause of persistent genital arousal disorder. Journal of Sexual Medicine, 11, 136-139. doi: 10.1111/jsm.12165

Bergeron, S., Khalife, S., Glazer, H. I., \& Binik, Y. M. (2008). Surgical and behavioral treatments for vestibulodynia: two-and-one-half year follow-up and predictors of outcome. Obstetrics \& Gynecology, 111(1), 159-166. doi:10.1097/01.AOG $.0000295864 .76032 . \mathrm{a} 7$

Bergeron, S., Rosen, N. O., \& Morin, M. (2011). Genital pain in women: Beyond interference with intercourse. Pain, 152, 1223-1225. doi:10.1016/j.pain.2011.01 .035

Bernard, A. L., Prince, A., \& Edsall, P. (2000). Quality of life issues for fibromyalgia patients. Arthritis Care and Research, 13(1), 42-50. doi:10.1002/15290131(200002)13:1<42::AID-ART7>3.0.CO;2-R 
Bolger, N., \& Amarel, D. (2007). Effects of social support visibility on adjustment to stress: Experimental evidence. Journal of Personality and Social Psychology, 92, 458-475. doi:10.1037/0022-3514.92.3.458

Brock, R. L., \& Lawrence, E. (2014). Intrapersonal, interpersonal, and contextual risk factors for overprovision of partner support in marriage. Journal of Family Psychology, 28(1), 54-64. doi:10.1037/a0035280

Brock, R. L., \& Lawrence, E. (2009). Too much of a good thing: Underprovision and overprovision of partner support. Journal of Family Psychology, 23(2), 181-192. doi:10.1037/a0015402

Byrne, D. \& Kelley, K. (Eds.) (1986). Alternative approaches to the study of sexual behavior. Hillsdale, NJ: Erlbaum.

Carvalho, J., Verissimo, A., \& Nobre, P. J. (2015). Psychological factors predicting the distress to female persistent genital arousal symptoms. Journal of Sex \& Marital Therapy. 41(1), 11-24. doi:10.1080/0092623X.2013.869776

Carvalho, J., Verissimo, A., \& Nobre, P. J. (2013). Cognitive and emotional determinants characterizing women with persistent genital arousal disorder. Journal of Sexual Medicine, 10, 1549-1558. doi:10.1111/jsm.12122

Cherner, R. A. \& Reissing, E. D. (2013). A psychological investigation of sexual arousal in women with lifelong vaginismus. Journal of Sexual Medicine, 10, 1291-1303. doi:10.1111/jsm.12102 
Chivers, M. L., Rieger, G., Latty, E., \& Bailey, J. M. (2004). A sex difference in the specificity of sexual arousal. Psychological Science, 15, 736-744. doi:10.1111/j.0956-7976.2004.00750.x

Collins, N. L., \& Feeney, B. C. (2004). Working models of attachment shape perceptions of social support: evidence from experimental and observational studies. Journal of Personality and Social Psychology, 87, 363-383. doi:10.1037/00223514.87.3.363

Cooley, M. E., Short, T. H., \& Moriarty, H. J. (2003). Symptom prevalence, distress, and change over time in adults receiving treatment for lung cancer. Psychooncology, 12(7), 694-708. doi:10.1002/pon.694

Crandall, C. S., \& Moriarty, D. (1995). Physical illness stigma and social rejection. British Journal of Social psychology, 34, 67-83. doi:10.1111/j.20448309.1995.tb01049.x

Creswell, J. W. (2014). Research design: Qualitative, quantitative, and mixed methods approaches (4th ed.). Thousand Oaks, CA: Sage.

Creswell, J. (2008). Educational research: Planning, conducting, and evaluating quantitative and qualitative research. New Jersey: Pearson; Merrill Prentice Hall.

Cyranowski, J. M., Bromberger, J., Youk, A., Matthews, K., Kravitz, D. O., \& Powell, L. H. (2004a). Lifetime depression history and sexual function in women at midlife. Archives of Sexual Behavior, 33, 539-548. doi:10.1023/B.ASEB.0000044738 $.84813 .3 b$ 
Cyranowski, J. M., Frank, E., Cherry, C., Houck, P., \& Kupfer, D. J. (2004b).

Prospective assessment of sexual function in women treated for recurrent major depression. Journal of Psychiatric Research, 38, 267-273. doi:10.1016/j .jpsychires.2003.08.003

Davis, C. M., Yarber, W. L., Bauserman, R., Schreer, G., Davis, S. L. (1998). Handbook of sexuality-related measurements. Thousand Oaks, CA: Sage Publications, Inc.

DeRogatis, L. R., Clayton, A. H., Goldstein, A., Lewis-D’Agostino, D., Wunderlich, G., \& Cotton, D. (2011). eDiary and female sexual distress scale in evaluating distress in hypoactive sexual desire disorder (HSDD). Journal of Sex Research, 48(6), 565-572. doi:10.1080/00224499.2010.524321

DeRogatis, L., Clayton, A., Lewis-D’Agostino, \& Wunderlich, G. (2008). Validation of the female sexual distress scale-revised for assessing distress in women with hypoactive sexual desire disorder. Journal of Sexual Medicine, 5(2), 357-364. doi:10.1111/j.1743-6109.2007.00672.x

Dewitte, M. (2012). Different perspectives on the sex-attachment link: Towards an emotion-motivational account. Journal of Sex Research, 49(2-3), 105-124. doi:10.1080/00224499.2011.576351

Dunn, K. M., Croft, P. R., \& Hackett, G. I. (1999). Association of sexual problems with social, psychological, and physical problems in men and women: a cross sectional population survey. Journal of Epidemiology and Community Health, 53(3), 144148. doi:10.1136/jech.53.3.144 
Eibye, S. \& Jensen, H. M. (2014). Persistent genital arousal disorder: Confluent patient history of agitated depression, paroxetine cessation, and a Tarlov cyst. Case Reports in Psychiatry, 2014, 1-4. doi:10.1155/2014/529052

Elkins, G. R., Ramsey, D., \& Yu, Y. (2014). Hypnotherapy for persistent genital arousal disorder: A case study. International Journal of Clinical and Experimental Hypnosis, 62(2), 215-223. doi:10.1080/00207144.2014.869136

Echenberg, R. (2011). Dr. Robert Echenberg answers questions and offers his own comments on PGAD. Retrieved August 24, 2016, from http://www.instituteforwomeninpain.com/dr.-echenberg-answers-questions-onpgad

Endler, N. S. (1997). Stress, anxiety, and coping: The multidimensional interaction model. Canadian Psychology, 38(3), 136-153. doi:10.1037/0708-5591.38.3.136

Engel, G. (1980). The clinical application of the biopsychosocial model. American Journal of Psychiatry, 137(5), 535-544. doi:10.1176/aip.137.5.535

Evans, D. T. (2000). Speaking of sex: the need to dispel myths and overcome fears. British Journal of Nursing, 9(10), 650-655. doi:10.12968/bjon.2000.9.10.6278

Facelle, T. M., Sadeghi-Nejad, H., \& Goldmeier, D. (2013). Persistent genital arousal disorder: Characterization, etiology, and management. Journal of Sexual Medicine, 10(2), 439-450. doi:10.1111/j.1743-6109.2012.02990.x.

Faul, F., Erdfelder, E., Buchner, A., \& Lang, A. G. (2009). Statistical power analysis using $\mathrm{G}^{*}$ Power 3.1: Tests for correlation and regression analyses. Behavior Research Methods, 41, 1149-1160. doi:10.3758/BRM.41.4.1149 
Field, A. (2013). Discovering Statistics Using IBM SPSS Statistics (4th ed.). London: Sage.

Fife, B. L. \& Wright, E. R. (2000). The dimensionality of stigma: A comparison of its impact on the self of persons with HIV/AIDS and Cancer. Journal of Health and Social Behavior, 41, 50-67. doi:10.2307/2676360

Fisher, W. A., Byrne, D., \& White, L. A. (1983). Emotional barriers to contraception. In D. Byrne \& W. A. Fisher (Eds.), Adolescents, sex, and contraception. Hillsdale, NJ: Lawrence Erlbaum.

Fisher, T. D., Davis, C. M., Yarber, W. L., \& Davis, S. L. (2013). Handbook of sexualityrelated measurements. ( $3^{\text {rd }}$ ed.). New York, NY: Routledge.

Fisher, W. A., White, L. A., Byrne, D., \& Kelley, K. (1988). Erotophobia-erotophilia as a dimension of personality. The Journal of Sex Research, 25(1), 123-151. doi:10.1080/00224498809551448

Frankfort-Nachmias, C., \& Nachmias, D. (2008). Research methods in the social sciences (7th ed.). New York: Worth.

Fried, E. I. \& Nesse, R. M. (2015). Depression sum-scores don’t add up: why analyzing specific depression symptoms is essential. BMC Medicine, 13 (72), 1-11. doi:10.1186/s12916-015-0325-4

Furnham, A. \& Hiranandani, M. (2009). Sexual attitudes and erotophobia and the recall of sexual content on television. Communications, 34, 73-86. doi:10.1515/COMM.2009.005 
Gadit, A. (2013). Persistent genital arousal disorder: a clinical challenge. BMJ Case Reports, 2013, 1-2. doi:10.1136/bcr-2013-009098

Garvey, L. J., West, C., Latch, N., Leiblum, S., \& Goldmeier, D. (2009). Report of spontaneous and persistent genital arousal in women attending a sexual health clinic. International Journal of STD \& AIDS, 20, 519-521. doi:10.1258/ijsa.2008.008492

Gilbert, F. S. \& Gamache, M. P. (1984). The sexual opinion survey: Structure and use. The Journal of Sex Research, 20(3), 293-309. doi:10.1080/00224498409551227

Girme, Y. U., Overall, N. C., Simpson, J. A., \& Fletcher, G. J. O. (2015). “All or nothing”: Attachment avoidance and the curvilinear effects of partner support. Journal of Personality and Social Psychology, 108(3), 450-475. doi:10.1037/a0038866

Gleason, M. E. J., Iida, M., Shrout, P. E., \& Bolger, N. (2008). Receiving support as a mixed blessing: Evidence for dual effects of support on psychological outcomes. Journal of Personality and Social Psychology, 94, 824-838. doi:10.1037/00223514.94.5.824

Goldmeier, D., \& Leiblum, S. (2008). Interaction of organic and psychological factors in persistent genital arousal disorder in women: A report of six cases. International Journal of STD \& AIDS, 19(7), 488-490. doi:10.1258/ijsa.2007.007298.

Goldmeier, D., Bell, C., \& Richardson, D. (2006). Withdrawal of selective serotonin reuptake inhibitors (SSRIs) may cause increased atrial natriuretic peptide and 
persistent sexual arousal in women? Journal of Sexual Medicine, 3, 376. doi:10.1111/j.1743-6109.2006.00224.x

Goldmeier, D., Mears, A., Hiller, J., \& Crowley, T. (2009). Persistent genital arousal disorder: A review of the literature and recommendations for management. International Journal of STD \& AIDS, 20, 373-377. doi:10.1258/ijsa.2009 .009087

Graziottin, A. (2004). Sexual arousal: similarities and differences between men and women. Journal of Men's Health \& Gender, 1(2-3), 215-223. doi:10.1016/j.mhg.2004.07.026

Green, Samuel B. \& Salkind, Neil, J. (2011). Using SPSS for Windows and Macintosh: Analyzing and understanding data ( $6^{\text {th }}$ Ed.). Upper Saddle River: NJ

Gunn, J. M., Ayton, D. R., Densley, K., Pallant, J. F., Chondros, P., Herrman, H. E., \& Dowrick, C. F. (2012). The association between chronic illness, multimorbidity and depressive symptoms in an Australian primary care cohort. Social Psychiatry and Psychiatric Epidemiology, 47(2), 175-184. doi:10.1007/s00127-010-0330-z

Hall, C. L., Hogue, T., \& Guo, K. (2014). Sexual cognition guides viewing strategies to human figures. Journal of Sex Research, 51(2), 184-196. doi:10.1080/00224499 .2012 .716872

Hamilton, L. D. \& Meston, C. M. (2013). Chronic stress and sexual function in women. Journal of Sexual Medicine, 10(10), 2443-2454. doi:10.1111/jsm.12249 
Hanley, N. R. \& Van de Kar, L. D. (2003). Serotonin and the neuroendocrine regulation of the hypothalamic-pituitary-adrenal axis in health and disease. Vitamins and Hormones, 66, 189-255. doi:10.1016/S0083-6729(03)01006-9

Hayes, A. (2012). Hayes, A. F. (2012). PROCESS: A versatile computational tool for observed variable mediation, moderation, and conditional process modeling [White paper]. Retrieved from http://www.afhayes.com/public/process2012.pdf

Helweg-Larsen, M. \& Howell, C. (2000). Effects of erotophobia on the persuasiveness of condom advertisements containing strong or weak arguments. Basic and Applied Social Psychology, 22(2), 111-117. doi:10.1207/15324830051036199

Hendrickx, L., Gijs, L., \& Enzlin, P. (2013). Distress, sexual dysfunctions, and DSM: Dialogue at cross purposes? Journal of Sexual Medicine, 10, 630-641. doi:10.1111/j.1743-6109.2012.02971.x

Henry, J. D. \& Crawford, J. R. (2005). The short-form version of the depression anxiety stress scales (DASS-21): Construct validity and normative data in a large nonclinical sample. British Journal of Clinical Psychology, 44, 227-239. doi:10.1348/014466505X29657

Holmbeck, G. (1997). Toward terminological, conceptual, and statistical clarity in the study of mediators and moderators: Examples from the child-clinical and pediatric psychology literatures. Journal of Consulting and Clinical Psychology, 65(4), 599-610. doi:10.1037//0022-006X.65.4.599

Kaplan, H. S. (1995). The sexual desires disorders: Dysfunctional regulation of the sexual motivation. New York, NY: Routledge Taylor \& Francis Group. 
Keane, T. M., Taylor, K. L., \& Penk, W. E. (1997). Differentiating post-traumatic stress disorder (PTSD) from major depression (MDD) and generalized anxiety disorder (GAD). Journal of Anxiety Disorders, 11(3), 317-328 doi:10.1016/S08876185(97)00013-3

Kendall-Tackett, K. (Ed.). (2010). The psychoneuroimmunology of chronic disease: Exploring the links between inflammation, stress, and illness. Washington, DC: American Psychological Association.

Kennedy, S. H., Dickens, S. E., Eisfeld, B. S., \& Bagsby, R. M. (1999). Sexual dysfunction before antidepressant therapy in major depression. Journal of Affective Disorders, 56, 201-208. doi:10.1016/S0165-0327(99)00050-6

King, S. A., Goldstein, I., \& Pfaus, J. (2016). Mechanism of action and preliminary clinical experience with zolpidem, a nonbenzodiazepine indirect GABA a receptor agonist, for symptomatic treatment of persistent genital arousal disorder (PGAD). Journal of Sexual Medicine, 13(6), S247-S248. doi:10.1016/j.jsxm .2016 .04 .020

Kinsinger, S. W., Laurenceau, J., Carver, C. S., \& Antoni, M. H. (2011). Perceived partner support and psychosexual adjustment to breast cancer. Psychology and Health, 26(12), 1571-1588. doi:10.1080/08870446.2010.533771

Knaster, P., Estlander, A., Karlsson, H., Kapria, J., \& Kalso, E. (2016). Diagnosing depression in chronic pain patients: DSM-IV major depressive disorder vs. Beck Depression Inventory (BDI). PLoS ONE, 11(3): e0151982.

doi:10.1371/journal.pone.0151982 
Komisaruk, B. R. \& Lee, H. (2012). Prevalence of sacra spinal (Tarlov) cysts in persistent genital arousal disorder. Journal of Sexual Medicine, 9, 2047-2056. doi:10.1111/j.1743-6109.2012.02765.x

Korda, J. B., Pfaus, J. G., Kellner, C. H., \& Goldstein, I. (2009). Persistent genital arousal disorder (PGAD): Case report of long-term symptomatic management with electroconvulsive therapy. Journal of Sexual Medicine, 6, 2901-2909. doi:10.1111/j.1743-6109.2009.01421.x

Krapf, J. M. \& Goldstein, A. (2013). Two case presentations of profound labial edema as a presenting symptom of hypermobility-type Ehlers-Danlos syndrome. Journal of Sexual Medicine, 10, 2347-2350. doi:10.1111/jsm.12229

Kuffel, S.W., \& Heiman, J. R. (2006). Effects of depressive symptoms and experimentally adopted schemas on sexual arousal and affect in sexually healthy women. Archives of Sexual Behavior, 35, 163-177. doi:10.1007/s10508-0059015-1

Laumann, E. O., \& Waite, L. J. (2008). Sexual dysfunction among older adults: Prevalence and risk factors from a nationally representative US probability sample of men and women 57-85 years of age. The journal of sexual medicine, 5(10), 2300-2311. doi:10.1111/j.1743-6109.2008.00974.x.

Laumann, E. O., Paik, A., \& Rosen, R. C. (1999). Sexual dysfunction in the United States: prevalence and predictors. Jama, 281(6), 537-544. doi:10.1001/jama.281.6.537

Lazarus, R. S. (1991). Emotion and adaptation. London: Oxford University Press. 
Leiblum, S. (2009). Persistent genital arousal disorder: An update of theory and practice. The Female Patient, 24, 19-20.

Leiblum, S. (2008). Persistent genital arousal disorder in women: What is known and what is unknown. Abstracts of the $9^{\text {th }}$ Congress of the European Federation of Sexology: Sexologies, 17(Supp 1), S26-S27). doi:10.1016/S1158-1360(08)725775

Leiblum, S., Brown, C., Wan, J., \& Rawlinson, L. (2005). Persistent sexual arousal syndrome: A descriptive study. Journal of Sexual Medicine. 2(3), 331-337. doi:10.1111/j.1743-6109-2005.20357.x

Leiblum, S. R., \& Chivers, M. L. (2007). Normal and persistent genital arousal in women: New perspectives. Journal of Sex and Marital Therapy, 33(4), 357-373. doi:10.1080/00926230701385605

Leiblum, S. R., \& Goldmeier, D. (2008). Persistent genital arousal disorder in women: Case reports of association with anti-depressant usage and withdrawal. Journal of Sex \& Marital Therapy, 34(2), 150-159. doi:10.1080/00926230701636205.

Leiblum, S. R. \& Nathan, S. G. (2001). Persistent sexual arousal syndrome: A newly discovered pattern of female sexuality. Journal of Sex \& Marital Therapy, 27(4), 365-380. doi:10.1080/009262301317081115

Leiblum, S., Seehuus, M., \& Brown, C. (2007). Persistent genital arousal: Disordered or normative aspect of female sexual response? Journal of Sexual Medicine, 4, 680689. doi:10.1111/j.1743-6109.2007.00495.x 
Leiblum, S. Seehuus, M., Goldmeier, D., \& Brown, C. (2007). Psychological, medical, and pharmacological correlates of persistent genital arousal disorder. Journal of Sexual Medicine, 4, 1358-1366. doi:10.1111/j.1743-6109.2007.00575.x

Lemieux, S. R., \& Byers, E. S. (2008). The sexual well-being of women who have experienced child sexual abuse. Psychology of Women Quarterly, 32(2), 126-144. doi:10.1111/j.1471-6402.2008.00418.x

Lewis, R. W., Fugl-Meyer, K. S., Corona, G., Hayes, R. D., Laumann, E. O., Moreira Jr, E. D., ... \& Segraves, T. (2010). Definitions/epidemiology/risk factors for sexual dysfunction. The journal of sexual medicine, 7(4pt2), 1598-1607. doi:10.1111/j.1743-6109.2010.01778.x.

Lovibond, P. F. \& Lovibond, S. H. (1995). The structure of negative emotional states: Comparison of the depression anxiety stress scales (DASS) with the bed depression and anxiety inventories. Behavioral Research and Therapy, 33(3), 335-343. doi:10.1016/0005-7967(94)00075-U

Lucena, H. M., Mukhopadkyay, S., \& Morris, E. (2015). Dyspareunia: a difficult symptom in gynaecological practice. Obstetrics, Gynaecology and Reproductive Medicine, 25(4), 96-101. doi:10.1016/j/ogrm.2015.01.007

Macapagal, K. R. \& Janssen, E. (2011). The valence of sex: Automatic affective associations in erotophilia and erotophobia. Personality and Individual Differences, S1(2011), 699-703. doi:10.1016/j.paid.2011.06.008 
Mahoney, S. \& Zarate Jr., C. (2007). Persistent sexual arousal syndrome: A case report and review of the literature. Journal of Sex \& Marital Therapy, 33, 65-71. doi:10.1080/00926230600998532

Markos, A. R. \& Dinsmore, W. (2013). Persistent genital arousal and restless genitalia: sexual dysfunction or subtype of vulvodynia? International Journal of STD \& AIDS, 24(11), 852-858. doi:10.1177/0956462413489276

McCabe, M., Althof, S. E., Assalian, P., Chevret-Measson, M., Leiblum, S. R., Simonelli, C. \& Wylie, K. (2010). Psychological and interpersonal dimensions of sexual function and dysfunction. Journal of Sexual Medicine, 7, 327-336. doi:10.1111/j.1743-6109.2009.01618.x

Meston, C. M., \& Bradford, A. (2007). Autonomic nervous system influences: the role of the sympathetic nervous system in female sexual arousal. In The Psychophysiology of Sex. (pp. 66-82). Bloomington, IN: Indiana University Press Meston, C. M., Rellini, A. H., \& Heiman, J. R. (2006). Women's history of sexual abuse, their sexuality, and sexual self-schemas. Journal of Consulting and Clinical Psychology, 74(2), 229. doi:10.1037/0022-006X.74.2.229

Mitchell, W. B., DiBartolo, P. M., Brown, T. A., \& Barlow, D. H. (1998). Effects of positive and negative mood on sexual arousal in sexually functional males.Archives of sexual behavior, 27(2), 197-207. doi:10.1023/A: 1018686631428 
Nestler, E. J., Hyman, S. E., \& Malenka, R. C. (2009). Molecular neuropharmacology: A foundation for clinical neuroscience (2nd Edition). China: McGraw-Hill Companies

Outcalt, S. D., Kroenke, K., Krebs, E. E., Chumbler, N. R., Wu, J., Yu, Z., \& Bair, M. J. (2015). Chronic pain and comorbid mental health conditions: independent associations of posttraumatic stress disorder and depression with pain, disability, and quality of life. Journal of Behavioral Medicine, 38, 535-543. doi:10.1007/s10865-015-9628-3

Peloquin K., Bigras, N., Brassard, A., \& Godbout, N. (2014). Perceiving that one’s partner is supportive moderates the associations among attachment insecurity and psychosexual variables. Canadian Journal of Human Sexuality, 23(3), 178-188. doi:10.3138/cjhs.2443

Pfaus, J. (2009). Pathways of sexual desire. Journal of Sexual Medicine, 6(6), 1506-1533. doi:10.1111/j.1743-6109.2009.01309.x

Philippsohn, S. \& Kruger, T. H. C. (2012). Persistent genital arousal disorder: Successful treatment with Duloxetine and Pregabalin in two cases. Journal of Sexual Medicine, 9, 213-217. doi:10.1111/j.1743-6109.2011.02518.x

Pink, L., Rancourt, V., \& Gordon, A. (2014). Persistent genital arousal in women with pelvic and genital pain. Journal of Obstetrics and Gynaecology Canada, 36(4), 324-330. doi:10.1016/S1701-2163(15)30608-3

Pink, L., Rancourt, V., \& Gordon, A. (2014). In response: To the editor. Journal of Obstetrics and Gynaecology Canada, 36(9), 765-766. 
Possover, M. \& Forman, A. (2012). Voiding dysfunction associated with pudendal nerve entrapment. Current Bladder Dysfunction Reports, 7(4), 281-285. doi:10.1007/s11884-012-0156-5

Puppo, V. (2014). Letter. Journal of Obstetrics and Gynaecology Canada, 36(9), 765.

Ramic, M. (2013). Case report: A case of persistent genital arousal disorder successfully treated with Topiramate in a physically healthy individual. Journal of Clinical Psychiatry, 74(7), 693. doi:10.4088/JCP.12cr08280

Restless Legs Syndrome. (2010). In Restless Legs Syndrome Fact Sheet: National Institute of Neurological Disorders and Stroke online. Retrieved from http://www.ninds.nih.gov/disorders/restless_legs/detail_restless_legs.htm

Rholes, W. S., Simpson, J. A., Kohn, J. L., Wilson, C. L., Martin, A. M., III, Tran, S., \& Kashy, D. A. (2011). Attachment orientations and depression: A longitudinal study of new parents. Journal of Personality and Social Psychology, 100, 567586. doi:10.1037/a0022802

Rosen, R., \& Leiblum, S. R. (Eds.). (1995). Case studies in sex therapy. Guilford Press. Rosen, R., Brown, C., Heiman, J., Leiblum, S., Meston, C., Shabsigh, R.,...D’Agostino Jr., R. (2000). The female sexual function index (FSFI): A multidimensional selfreport instrument for the assessment of female sexual function. Journal of Sex \& Marital Therapy, 26, 191-208. doi:10.1080/009262300278597

Rosenbaum, T. Y. (2010). Physical therapy treatment of persistent genital arousal disorder during pregnancy: A case report. Journal of Sexual Medicine, 7(3), 13061310. doi:10.1111/j.1743-6109.2009.01654.x 
Runels, C., Melnick, H., Debourbon, E., \& Roy, L. (2014). A pilot study of the effect of localized injections of autologous platelet rich plasma (PRP) for the treatment of female sexual dysfunction. Journal of Women's Health Care, 3(4), 1-4. doi.10.4172/2167-0420.1000169

Sawin, E. M. (2012). “The body gives way, things happen”: Older women describe breast cancer with a non-supportive intimate partner. European Journal of Oncology Nursing, 16, 64-70. doi:10.1016/j.ejon.2011.03.006

Schulz, U. \& Schwarzer, R. (2003). Social support in coping with illness: The Berlin Social Support Scales (BSSS). Diagnostica, 49, 73-82.

Schwarzer, R. (1996). Thought control of action: Interfering self-doubts. In I. G. Sarason, G. R. Pierce, \& B. R. Sarason (Eds.), Cognitive interference: Theories, methods, and findings (pp. 99-115). Mahwah, NJ: Erlbaum.

Shifren, J. L., Monz, B. U., Russo, P. A., Segreti, A., \& Johannes, C. B. (2008). Sexual problems and distress in United States women: prevalence and correlates. Obstetrics \& Gynecology, 112(5), 970-978. doi:10.1097/AOG $.0 \mathrm{~b} 013 \mathrm{e} 3181898 \mathrm{cdb}$

Simpson, J. A., Winterheld, H. A., Rholes, W. S., \& Orina, M. M. (2007). Working models of attachment and reactions to different forms of caregiving from romantic partners. Journal of Personality and Social Psychology, 93, 466-477. doi:10.1037/0022-3514.93.3

Smith, G. E. \& Nave, C. S. (2007). Illness transmission mode and perceiver personality: Factors affecting stigmatized perceptions of patients and sexual illness. Social 
Behavior and Personality Research, 35(6), 853-860. doi:10.2224/sbp.2007 .35 .6 .853

Smith, G. E., Hedges, T. J., Gerrard, M., \& Gibbons, F. X. (1996). Sexual attitudes, cognitive associative networks, and perceived vulnerability to unplanned pregnancy. Journal of Research in Personality, 30, 88-102. doi:10.1006/jrpe .1996 .0006

Spielberger, C. D. (1980). Test Anxiety Inventory: Preliminary professional manual. Palo Alto, CA: Consulting Psychologists Press.

Spielberger, C. D. (1983). Manual for the State-Trait Anxiety Inventory (STAI). PaloAlto, CA: Consulting Psychologists Press.

Stevenson, B. J. \& Kohler, T. (2015). First reported case of isolated persistent genital arousal disorder in a male. Case Reports in Urology, 2015(465748), 1-3. doi:10.1155/2015/465748

Stress [Def. 2]. (2015.). Oxford Dictionaries Online. In Oxford Dictionairies. Retrieved September 20, 2015, from http://www.oxforddictionaries.com/us/definition/american_english/stress.

Thorne, C. \& Stuckey, B. (2007). Pelvic congestion syndrome presenting as persistent genital arousal: A case report. Journal of Sexual Medicine, 5, 504-508. doi:10.1111/j.1743-6109.2007.00666.x

Waldinger, M. D. \& Schweitzer, D. H. (2009). Persistent genital arousal disorder in 18 Dutch women: Part II - A syndrome clustered with restless legs and overactive 
bladder. Journal of Sexual Medicine, 6, 482-497. doi:10.1111/j.1743-

6109.2008.01114.x

Waldinger, M. D., de Lint, G. J., Venema, P. L., van Gils, A. P. G., \& Schweitzer, D. H. (2010). Successful transcutaneous electrical nerve stimulation in two women with restless genital syndrome: The role of Ad- and C-nerve fibers. Journal of Sexual Medicine, 7, 1190-1199. doi:10.1111/j.1743-6109.2009.01578.x

Waldinger, M. D., van Gils, A. P. G., Ottervanger, H. P., Vandenbroucke, W. V., \& Tavy, D. L. (2009). Persistent genital arousal disorder in 18 Dutch women: Part I. MRI, EEG, and transvaginal ultrasonography investigations. Journal of Sexual Medicine, 6(2), 474-481. doi:10.1111/j.1743-6109.2008.01113.x.

Waldinger, M. D., Venema, P. L., van Gils, A. P. G., de Lint, G. J., \& Schweitzer, D. H. (2011). Stronger evidence for small fiber sensory neuropathy in restless genital syndrome: two case reports in males. Journal of Sexual Medicine, 8(1), 325-330. doi:10.1111/j.1743-6109.2010.02079.x

Waldinger, M.D., Venema, P. L., van Gils, A. P. G., Schutter, E. M. J., \& Schweitzer, D. H. (2010). Restless genital syndrome before and after clitoridectomy for spontaneous orgasms: A case report. Journal of Sexual Medicine, 7, 1029-1034. doi:10.1111/j.1743-6109.2009.01571.x

Waldinger, M. D., Venema, P. L., van Gils, A. P. G., \& Schweitzer, D. H. (2009). New insights into restless genital syndrome: Static mechanical hyperesthesia and neuropathy of the nervus dorsalis clitoridis. Journal of Sexual Medicine, 6, 27782787. doi:10.1111/j.1743-6109.2009.01435.X 
Wiegel, M., Scepkowski, L. A., \& Barlow, D. H. (2006). Cognitive and affective processes in female sexual dysfunctions. In I. Goldstein, C. Meston, S. Davis, \& A. Traish (Eds.), Women's sexual function and dysfunction: Study, diagnosis and treatment (pp. 85-92). Abingdon, Oxon: Taylor \& Francis.

Wilson, H., Mannix, S., Oko-osi, H., \& Revicki, D. A. (2015). The impact of medication on health-related quality of life in patients with generalized anxiety disorder. CNS Drugs, 29, 29-40. doi:10.1007/s40263-014-0217-8

Yildirim, E. A., Hacioglu, M., Essizoglu, A., \& Kucukparlak, I. (2012). Persistent genital arousal disorder misdiagnosed because of Islamic religious bathing rituals: A report of three cases. Journal of Sex \& Marital Therapy, 38, 436-444. doi:10.1080/0092623X.2011.606888 
Appendix A: Scales and Questionnaires

DEMOGRAPHICS

1. Did you receive this survey invitation from?

(Select only one.)

$$
\begin{aligned}
& 1 \text { - an email from San Diego Sexual Medicine } \\
& 2 \text { - Facebook }{ }^{\mathrm{TM}} \text { support group }
\end{aligned}
$$

2. What is your age?

(Enter age)

3. What country do you reside in?

(Enter country)

4. What is your race?

(Select only one.)

$$
\begin{aligned}
& \text { 1- Caucasian } \\
& 2 \text { - African American/Black } \\
& 3 \text { - Asian } \\
& 4 \text { - Native American/Alaska Native } \\
& 5 \text { - Native Hawaiin/Other Pacific Islander } \\
& 6 \text { - Latino/a } \\
& 7 \text { - Multiracial (please specify) } \\
& 8 \text { - Other (please specify) }
\end{aligned}
$$

5. What is the highest level of education you have completed? (Select only one.)

$$
\begin{aligned}
& 1 \text { - High School Diploma } \\
& 2 \text { - Associates degree } \\
& 3 \text { - Bachelor's degree } \\
& 4 \text { - Master's degree } \\
& 5 \text { - Doctorate degree } \\
& 6 \text { - Other (please specify) }
\end{aligned}
$$

6. How would you describe your current Religious affiliation?

(Select only one.)

$$
\begin{array}{ll}
\square & 1 \text { - Christian } \\
\square & 2 \text { - Jewish } \\
\square & 3 \text { - Muslim } \\
\square & 4 \text { - Buddhist } \\
\square & 5 \text { - Hindu } \\
\square & 6 \text { - Sikh } \\
\square \quad 7 \text { - Atheist / Agnostic }
\end{array}
$$


8 - No religious affiliation

9 - Other (please specify)

7. To what extent, if at all, do you consider yourself religious (an organized systems of worship that gives a framework to the relationship we have with the universe and with a higher power)?

(Select only one.)

1 - Not at all Religious

2 - Slightly Religious

3 - Somewhat Religious

4 - Quite Religious

5 - Extremely Religious

8. To what extent, if at all, do you consider yourself spiritual (a sense of connection to the universe and to a higher power)?

(Select only one.)

1 - Not at all Spiritual

2 - Slightly Spiritual

3 - Somewhat Spiritual

4 - Quite Spiritual

5 - Extremely Spiritual

9. What is your employment status?

1 - Full time employed

2 - Part time employed

3 - Student

4 - Retired

5 - Non employed (i.e. - choose to stay home, in-between jobs, on disability)

10. What is your household income?

(Select only one.)

$$
\begin{aligned}
& 1-<\$ 25,000 \text { per year } \\
& 2 \text { - } \$ 25,001-\$ 50,000 \text { per year } \\
& 3 \text { - } \$ 50,001-\$ 75,000 \text { per year } \\
& 4-\$ 75,001-\$ 100,000 \text { per year } \\
& 5 \text { - }>\$ 100,000 \text { per year }
\end{aligned}
$$

11 . What is your relationship status?

\section{(Select only one.)}

1 - Single

2 - Married

3 - Separated

4 - Divorced

5 - In a relationship, live with partner

6 - In a relationship, but not living with partner 
12. Over our lifetime, sexuality is experienced as both psychological (thoughts, fantasy) and behavioral (activities we engage in) expression. We often fantasize about activity that we never wish to, nor will actually engage in. Rate yourself, in terms of your own fantasies over the course of your life, on the following scale:

\section{(Select only one.)} sex)

1 - Exclusively heterosexual (opposite sex), with no homosexual (same

2 - Predominantly heterosexual, with only rare / incidental homosexual

3 - Predominantly heterosexual, but more than incidental homosexual

4 - Equally heterosexual and homosexual

5 - Predominantly homosexual, but more than incidental homosexual

6 - Predominantly homosexual, with only rare / incidental homosexual

7 - Exclusively homosexual, with no heterosexual

13. Rate yourself, in terms of your own behavior with partners over the course of your life, on the following scale:

\section{(Select only one.)} sex)

1 - Exclusively heterosexual (opposite sex), with no homosexual (same

2 - Predominantly heterosexual, with only rare / incidental homosexual

3 - Predominantly heterosexual, but more than incidental homosexual

4 - Equally heterosexual and homosexual

5 - Predominantly homosexual, but more than incidental homosexual

6 - Predominantly homosexual, with only rare / incidental homosexual

7 - Exclusively homosexual, with no heterosexual 


\section{GYNECOLOGICAL AND MEDICAL HISTORY}

1. Please clarify which of the following symptoms you have experienced within the past six months:

\section{(Select all that apply.)}

$\square \quad 1$ - Persistent feelings of arousal extending over days or weeks or months

2 - The arousal is not relieved by or only temporarily relieved by $\operatorname{orgasm}(\mathrm{s})$

3 - The arousal is not associated with sexual thoughts or desire

4 - The arousal may be triggered by nonsexual stimuli or no apparent stimuli

5 - The arousal is bothersome and causes distress

2. How long have you experienced symptoms of PGAD?

Number of months

Number of years

3. During the past six months, how distressed have you been regarding your PGAD symptoms?

(Select only one.)

0 - Not at all distressed

1 - Slightly distressed

2 - Somewhat distressed

3 - Quite distressed

4 - Extremely distressed

4. During the past six months, how embarrassed have you been regarding your PGAD symptoms?

(Select only one.)

0 - Not at all embarrassed

1 - Slightly embarrassed

2 - Somewhat embarrassed

3 - Quite embarrassed

4 - Extremely embarrassed

5. Have you visited a health care provider regarding your PGAD symptoms?

(Select only one.)

$\square \quad 1$ - Yes

2 - No

6. How many separate health care providers have you seen regarding your PGAD symptoms?

(Select only one.)

0 - I have not seen a health care provider for my PGAD symptoms 


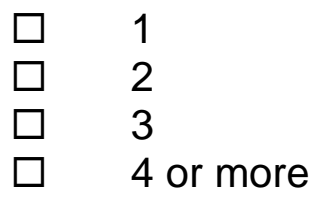

7. Have you received a diagnosis of PGAD from a health care provider (Select only one.)

1 - Yes

2 - No

8. Have you been satisfied with the support you have received from health care providers regarding your PGAD symptoms?

(Select only one.)

$$
\begin{aligned}
& 1 \text { - Not at all satisfied } \\
& 2 \text { - Slightly satisfied } \\
& 3 \text { - Somewhat satisfied } \\
& 4 \text { - Quite satisfied } \\
& 5 \text { - Extremely satisfied } \\
& 6 \text { - Other, please explain }
\end{aligned}
$$

9. Are you currently?

(Select only one.)

$\begin{array}{ll}\square & 1 \text { - Pre-menopausal } \\ \square & 2 \text { - Peri-menopausal } \\ \square & 3 \text { - Post-menopausal } \\ \square & 4 \text { - Unknown }\end{array}$

10. How many times have you been pregnant? (Select only one.)

$\begin{array}{ll}\square & 0 \\ \square & 1 \\ \square & 2 \\ \square & 3 \\ \square & 4 \text { or more }\end{array}$

11. To how many children have you given birth? (Select only one.)

$\begin{array}{ll}\square & 0 \\ \square & 1 \\ \square & 2 \\ \square & 3 \\ \square & 4 \text { or more }\end{array}$

12. Did you have vaginal births or cesarean sections?

$\square \quad 1$ - Vaginal 
2 - Cesarean

3 - Both

4 - Neither - currently pregnant with first child

5 - Not Applicable - no children or have adopted/surrogate/partners children

13. Have you ever been diagnosed with Overactive Bladder?

$$
\begin{aligned}
& 1 \text { - Yes } \\
& 2 \text { - No }
\end{aligned}
$$

14. Have you ever been diagnosed with Restless Leg Syndrome?

$$
\begin{aligned}
& 1 \text { - Yes } \\
& 2 \text { - No }
\end{aligned}
$$

15. Have you ever been diagnosed with Tarlov Cyst(s)?

$$
\begin{array}{ll}
\square \quad 1-Y e s \\
\square \quad 2-\text { No }
\end{array}
$$

16. Have you ever been diagnosed with Pelvic Congestion Syndrome?

$\begin{array}{ll}\square & 1 \text { - Yes } \\ \square & 2-\mathrm{No}\end{array}$

17. Have you ever been diagnosed with Pudendal Neuropathy?

$$
\begin{array}{ll}
\square \quad 1 \text { - Yes } \\
\square \quad 2 \text { - No }
\end{array}
$$

18. Have you ever been diagnosed with a Sexually Transmitted Infection?

$$
\begin{aligned}
& 1 \text { - Yes } \\
& 2 \text { - No }
\end{aligned}
$$

The following questions are regarding prescription medications.

19. Are you currently or have you taken in the past any of the following SSRI's (selective serotonin reuptake inhibitor)/SNRI's (selective norepinephrine reuptake inhibitor)?

(Select all that apply)

$\begin{array}{rlcc} & \text { NAME } & \text { CURRENTLY } & \text { IN THE PAST } \\ 1 \square & \text { citalopram (Celexa) } & \square & \square \\ 2 \square & \text { escitalopram (Lexapro) } & \square & \square \\ 3 \square & \text { fluoxetine (Prozac) } & \square & \square \\ 4 \square & \text { fluvoxamine (Luvox) } & \square & \square \\ 5 \square & \text { paroxetine (Paxil) } & \square & \square \\ 6 \square & \text { sertraline (Zoloft) } & \square & \square \\ 7 \square & \text { desvenlafaxine (Pristiq) } & \square & \square \\ 8 \square & \text { duloxetine (Cymbalta) } & \square & \square \\ 9 \square & \text { venlafaxine (Effexor) } & \square & \square \\ 10 \square & \text { milnacipran (Savella) } & \square & \square \\ 11 \square & \text { levomilnacipran (Fetzima) } & \square & \square\end{array}$


20. Are you currently or have you taken in the past any of the following other Antidepressants?

(Select all that apply)

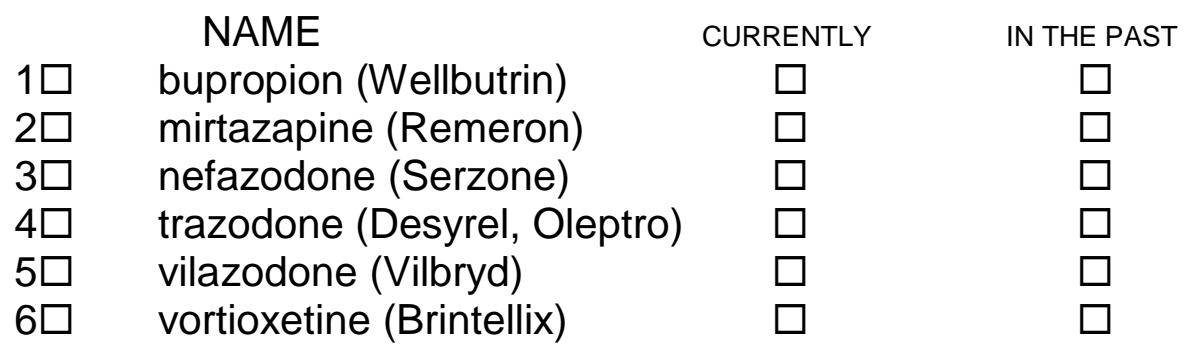

21. Do you associate the onset of your PGAD symptoms with any specific event (for example, stopping a medication)?

$\square \quad 1$ - Yes

$\square \quad 2-\mathrm{No}$

22. If you answered yes to question 21, please specify what event?

Comment: 
FSDS-R

FEMALE SEXUAL DISTRESS SCALE

(Revised-2005)

\section{INSTRUCTIONS}

Below is a list of feelings and problems that women sometimes have concerning their sexuality. Please read each item carefully, and circle the number that best describes HOW OFTEN THAT PROBLEM HAS BOTHERED YOU OR CAUSED YOU DISTRESS DURING THE PAST 7 DAYS I NCLUDI NG TODAY. Check only one number for each item, and take care not to skip any items.

Example: How often did you feel: Personal responsibility for your sexual problems.

\begin{tabular}{lllll} 
NEVER & RARELY & OCCASIONALLY & FREQUENTLY & ALWAYS \\
\hline & 1
\end{tabular}

\section{HOW OFTEN DI D YOU FEEL:}

1. Distressed about your sex life

2. Unhappy about your sexual relationship

3. Guilty about sexual difficulties

4. Frustrated by your sexual problems

5. Stressed about sex

6. Inferior because of sexual problems

7. Worried about sex

8. Sexually inadequate

9. Regrets about your sexuality

10. Embarrassed about sexual problems

11. Dissatisfied with your sex life

12. Angry about your sex life

13. Bothered by low sexual desire

$\begin{array}{lllll}0 & 1 & 2 & 3 & 4 \\ 0 & 1 & 2 & 3 & 4 \\ 0 & 1 & 2 & 3 & 4 \\ 0 & 1 & 2 & 3 & 4 \\ 0 & 1 & 2 & 3 & 4 \\ 0 & 1 & 2 & 3 & 4 \\ 0 & 1 & 2 & 3 & 4 \\ 0 & 1 & 2 & 3 & 4 \\ 0 & 1 & 2 & 3 & 4 \\ 0 & 1 & 2 & 3 & 4 \\ 0 & 1 & 2 & 3 & 4 \\ 0 & 1 & 2 & 3 & 4 \\ 0 & 1 & 2 & 3 & 4\end{array}$

Copyright @ 2000 by American Foundation for Urological Disease Inc. 
Actually Received Support, Recipient

Think about your current romantic partner. How did this person react to you during this past week? Please circle one response for each question.

\begin{tabular}{|c|c|c|c|c|}
\hline Question & $\begin{array}{l}\text { Strongly } \\
\text { Disagree }\end{array}$ & $\begin{array}{l}\text { Somewhat } \\
\text { Disagree }\end{array}$ & $\begin{array}{l}\text { Somewhat } \\
\text { Agree }\end{array}$ & $\begin{array}{l}\text { Strongly } \\
\text { Agree }\end{array}$ \\
\hline $\begin{array}{l}\text { 1. The person showed me that he/she } \\
\text { loves and accepts me. }\end{array}$ & 1 & 2 & 3 & 4 \\
\hline $\begin{array}{l}\text { 2. This person comforted me when I was } \\
\text { feeling bad. }\end{array}$ & 1 & 2 & 3 & 4 \\
\hline 3. This person left me alone. & 1 & 2 & 3 & 4 \\
\hline $\begin{array}{l}\text { 4. This person did not show much } \\
\text { empathy for my situation. }\end{array}$ & 1 & 2 & 3 & 4 \\
\hline 5. This person criticized me. & 1 & 2 & 3 & 4 \\
\hline $\begin{array}{l}\text { 6. This person made me feel valued and } \\
\text { important. }\end{array}$ & 1 & 2 & 3 & 4 \\
\hline $\begin{array}{l}\text { 7. This person expressed concern about } \\
\text { my condition. }\end{array}$ & 1 & 2 & 3 & 4 \\
\hline $\begin{array}{l}\text { 8. This person assured me that I can rely } \\
\text { completely on him/her. }\end{array}$ & 1 & 2 & 3 & 4 \\
\hline $\begin{array}{l}\text { 9. This person encouraged me not to } \\
\text { give up. }\end{array}$ & 1 & 2 & 3 & 4 \\
\hline $\begin{array}{l}\text { 10. This person was there when I needed } \\
\text { him/her. }\end{array}$ & 1 & 2 & 3 & 4 \\
\hline $\begin{array}{l}\text { 11. This person took care of many things } \\
\text { for me. }\end{array}$ & 1 & 2 & 3 & 4 \\
\hline $\begin{array}{l}\text { 12. This person took care of things I } \\
\text { could not manage on my own. }\end{array}$ & 1 & 2 & 3 & 4 \\
\hline $\begin{array}{l}\text { 13. This person helped me find something } \\
\text { positive in my situation. }\end{array}$ & 1 & 2 & 3 & 4 \\
\hline $\begin{array}{l}\text { 14. This person suggested activities that } \\
\text { might distract me. }\end{array}$ & 1 & 2 & 3 & 4 \\
\hline
\end{tabular}

\section{Satifaction with Support Received}

In general, I am very satifisfied with the way this person behaved. 
DASS 21

Please read each statement and circle a number 0,1 , 2, or 3 which indicates how much the statement applied to you over the past week. There are no right or wrong answers. Do not spend too much time on any statement.

The rating scale is as follows:

0 - Did not apply to me at all - NEVER

1 - Applied to me to some degree, or some of the time - SOMETIMES

2 - Applied to me to a considerable degree, or a good part of the time - OFTEN

3 - Applied to me very much, or most of the time - ALMOST ALWAYS

\begin{tabular}{|c|c|c|c|c|}
\hline & NEVER & SOMETIMES & OFTEN & $\begin{array}{l}\text { ALMOST } \\
\text { ALWAYS }\end{array}$ \\
\hline 1. I found it hard to wind down & 0 & 1 & 2 & 3 \\
\hline 2. I was aware of dryness of my mouth & 0 & 1 & 2 & 3 \\
\hline $\begin{array}{l}\text { 3. I couldn't seem to experience any } \\
\text { positive feeling at all }\end{array}$ & 0 & 1 & 2 & 3 \\
\hline $\begin{array}{l}\text { 4. I experienced breathing difficulty (eg, } \\
\text { excessively rapid breathing, } \\
\text { breathlessness in the absence of } \\
\text { physical exertion) }\end{array}$ & 0 & 1 & 2 & 3 \\
\hline $\begin{array}{l}\text { 5. I found it difficult to work up the } \\
\text { initiative to do things }\end{array}$ & 0 & 1 & 2 & 3 \\
\hline 6. I tended to over-react to situations & 0 & 1 & 2 & 3 \\
\hline $\begin{array}{l}\text { 7. I experienced trembling (eg, in the } \\
\text { hands) }\end{array}$ & 0 & 1 & 2 & 3 \\
\hline $\begin{array}{l}\text { 8. I felt that I was using a lot of nervious } \\
\text { energy }\end{array}$ & 0 & 1 & 2 & 3 \\
\hline $\begin{array}{l}\text { 9. I was worried about situations in } \\
\text { which I might panic and make a fool } \\
\text { of myself }\end{array}$ & 0 & 1 & 2 & 3 \\
\hline $\begin{array}{l}\text { 10. I felt that I had nothing to look } \\
\text { forward to }\end{array}$ & 0 & 1 & 2 & 3 \\
\hline 11. I found myself getting agitated & 0 & 1 & 2 & 3 \\
\hline 12. I found it difficult to relax & 0 & 1 & 2 & 3 \\
\hline 13. I felt down-hearted and blue & 0 & 1 & 2 & 3 \\
\hline 14. I was intolerant of anything that kept & 0 & 1 & 2 & 3 \\
\hline
\end{tabular}




\begin{tabular}{|l|c|c|c|c|}
\hline \multicolumn{1}{|c|}{$\begin{array}{l}\text { me from getting on with what I was } \\
\text { doing }\end{array}$} & & & \\
\hline 15. I felt I was close to panic & 0 & 1 & 2 & 3 \\
\hline $\begin{array}{l}\text { 16. I was unable to become enthusiastic } \\
\text { about anything }\end{array}$ & 0 & 1 & 2 & 3 \\
\hline 17. I felt I wasn't worth much as a person & 0 & 1 & 2 & 3 \\
\hline $\begin{array}{l}\text { 18. I felt that I was rather touchy } \\
\begin{array}{l}\text { 19. I was aware of the action of my heart } \\
\text { in the absence of physcial exertion } \\
\text { (eg, sense of heart rate increase, heart } \\
\text { missing a beat) }\end{array}\end{array}$ & 0 & 1 & 2 & 3 \\
\hline 20. I felt scared without any good reason & 0 & 1 & 2 & 3 \\
\hline 21. I felt that life was meaningless & 0 & 1 & 2 & 3 \\
\hline
\end{tabular}


Sexual Opinion Survey

Please respond to each item as honestly as you can. There are no right or wrong answers and your responses will be completely anonymous.

\section{I think it would be very entertaining to look at hard-core erotica.}

$$
\begin{aligned}
& 1=\text { strongly agree } \\
& 2=\text { agree } \\
& 3=\text { somewhat agree } \\
& 4=\text { neutral } \\
& 5=\text { somewhat disagree } \\
& 6=\text { disagree } \\
& 7=\text { strongly disagree }
\end{aligned}
$$

2. Erotica is obviously filthy and people should not try to describe it as anything else.

$$
\begin{aligned}
& 1=\text { strongly agree } \\
& 2=\text { agree } \\
& 3=\text { somewhat agree } \\
& 4=\text { neutral } \\
& 5=\text { somewhat disagree } \\
& 6=\text { disagree } \\
& 7=\text { strongly disagree }
\end{aligned}
$$

3. Swimming in the nude with a member of the opposite sex (with a member of my sexual interest) would be an exciting experience.

$$
\begin{aligned}
& 1=\text { strongly agree } \\
& 2=\text { agree } \\
& 3=\text { somewhat agree } \\
& 4=\text { neutral } \\
& 5=\text { somewhat disagree } \\
& 6=\text { disagree } \\
& 7=\text { strongly disagree }
\end{aligned}
$$

\section{Masturbation can be an exciting experience.}

$$
\begin{aligned}
& 1=\text { strongly agree } \\
& 2=\text { agree } \\
& 3=\text { somewhat agree } \\
& 4=\text { neutral } \\
& 5=\text { somewhat disagree } \\
& 6=\text { disagree }
\end{aligned}
$$


7 = strongly disagree

5. If I found out that a close friend of mine was homosexual, it would annoy me.

$$
\begin{aligned}
& 1=\text { strongly agree } \\
& 2=\text { agree } \\
& 3=\text { somewhat agree } \\
& 4=\text { neutral } \\
& 5=\text { somewhat disagree } \\
& 6=\text { disagree } \\
& 7=\text { strongly disagree }
\end{aligned}
$$

6. If people thought I was interested in oral sex, I would be embarrassed.

$$
\begin{aligned}
& 1=\text { strongly agree } \\
& 2=\text { agree } \\
& 3=\text { somewhat agree } \\
& 4=\text { neutral } \\
& 5=\text { somewhat disagree } \\
& 6=\text { disagree } \\
& 7=\text { strongly disagree }
\end{aligned}
$$

7. Engaging in group sex is an entertaining idea.

$$
\begin{aligned}
& 1=\text { strongly agree } \\
& 2=\text { agree } \\
& 3=\text { somewhat agree } \\
& 4=\text { neutral } \\
& 5=\text { somewhat disagree } \\
& 6=\text { disagree } \\
& 7=\text { strongly disagree }
\end{aligned}
$$

8. I personally find that thinking about engaging in sexual intercourse is arousing.

$$
\begin{aligned}
& 1=\text { strongly agree } \\
& 2=\text { agree } \\
& 3=\text { somewhat agree } \\
& 4=\text { neutral } \\
& 5=\text { somewhat disagree } \\
& 6=\text { disagree } \\
& 7=\text { strongly disagree }
\end{aligned}
$$


9. Seeing an erotic movie would be sexually arousing to me.

$$
\begin{aligned}
& 1=\text { strongly agree } \\
& 2=\text { agree } \\
& 3=\text { somewhat agree } \\
& 4=\text { neutral } \\
& 5=\text { somewhat disagree } \\
& 6=\text { disagree } \\
& 7=\text { strongly disagree }
\end{aligned}
$$

10. Thoughts that I might have homosexual tendencies would not worry me at all.

$$
\begin{aligned}
& 1=\text { strongly agree } \\
& 2=\text { agree } \\
& 3=\text { somewhat agree } \\
& 4=\text { neutral } \\
& 5=\text { somewhat disagree } \\
& 6=\text { disagree } \\
& 7=\text { strongly disagree }
\end{aligned}
$$

11. The idea of my being physically attracted to members of the same sex is not depressing.

$$
\begin{aligned}
& 1=\text { strongly agree } \\
& 2=\text { agree } \\
& 3=\text { somewhat agree } \\
& 4=\text { neutral } \\
& 5=\text { somewhat disagree } \\
& 6=\text { disagree } \\
& 7=\text { strongly disagree }
\end{aligned}
$$

\section{Almost all erotic material is nauseating.}

$$
\begin{aligned}
& 1=\text { strongly agree } \\
& 2=\text { agree } \\
& 3=\text { somewhat agree } \\
& 4=\text { neutral } \\
& 5=\text { somewhat disagree } \\
& 6=\text { disagree } \\
& 7=\text { strongly disagree }
\end{aligned}
$$


13. It would be emotionally upsetting to me to see someone exposing themselves publicly.

$$
\begin{aligned}
& 1=\text { strongly agree } \\
& 2=\text { agree } \\
& 3=\text { somewhat agree } \\
& 4=\text { neutral } \\
& 5=\text { somewhat disagree } \\
& 6=\text { disagree } \\
& 7=\text { strongly disagree }
\end{aligned}
$$

14. Watching a stripper of the opposite sex would not be very exciting.

$$
\begin{aligned}
& 1=\text { strongly agree } \\
& 2=\text { agree } \\
& 3=\text { somewhat agree } \\
& 4=\text { neutral } \\
& 5=\text { somewhat disagree } \\
& 6=\text { disagree } \\
& 7=\text { strongly disagree }
\end{aligned}
$$

\section{I would not enjoy seeing an erotic movie.}

$$
\begin{aligned}
& 1=\text { strongly agree } \\
& 2=\text { agree } \\
& 3=\text { somewhat agree } \\
& 4=\text { neutral } \\
& 5=\text { somewhat disagree } \\
& 6=\text { disagree } \\
& 7=\text { strongly disagree }
\end{aligned}
$$

16. When I think about seeing pictures showing someone of the same sex as myself masturbating, it nauseates me.

$$
\begin{aligned}
& 1=\text { strongly agree } \\
& 2=\text { agree } \\
& 3=\text { somewhat agree } \\
& 4=\text { neutral } \\
& 5=\text { somewhat disagree } \\
& 6=\text { disagree } \\
& 7=\text { strongly disagree }
\end{aligned}
$$


17. The thought of engaging in unusual sex practices is highly arousing.

$$
\begin{aligned}
& 1=\text { strongly agree } \\
& 2=\text { agree } \\
& 3=\text { somewhat agree } \\
& 4=\text { neutral } \\
& 5=\text { somewhat disagree } \\
& 6=\text { disagree } \\
& 7=\text { strongly disagree }
\end{aligned}
$$

18. Manipulating my genitals would probably be an arousing experience.

$$
\begin{aligned}
& 1=\text { strongly agree } \\
& 2=\text { agree } \\
& 3=\text { somewhat agree } \\
& 4=\text { neutral } \\
& 5=\text { somewhat disagree } \\
& 6=\text { disagree } \\
& 7=\text { strongly disagree }
\end{aligned}
$$

19. I do not enjoy daydreaming about sexual matters.

$$
\begin{aligned}
& 1=\text { strongly agree } \\
& 2=\text { agree } \\
& 3=\text { somewhat agree } \\
& 4=\text { neutral } \\
& 5=\text { somewhat disagree } \\
& 6=\text { disagree } \\
& 7=\text { strongly disagree }
\end{aligned}
$$

\section{I am curious about explicit erotica.}

$$
\begin{aligned}
& 1=\text { strongly agree } \\
& 2=\text { agree } \\
& 3=\text { somewhat agree } \\
& 4=\text { neutral } \\
& 5=\text { somewhat disagree } \\
& 6=\text { disagree } \\
& 7=\text { strongly disagree }
\end{aligned}
$$


21. The thought of having long-term sexual relations with more than one sex partner is not disgusting to me.

$$
\begin{aligned}
& 1=\text { strongly agree } \\
& 2=\text { agree } \\
& 3=\text { somewhat agree } \\
& 4=\text { neutral } \\
& 5=\text { somewhat disagree } \\
& 6=\text { disagree } \\
& 7=\text { strongly disagree }
\end{aligned}
$$




\section{Open-Ended Questions}

1. Please describe what ways, if any, PGAD has affected your life.

2. What aspects of PGAD, if any, do you feel have most impacted your quality of life? 
Appendix B: Email Invitation to Participate

As a client of San Diego Sexual Medicine and a woman over the age of 17 who has been suffering from Persistent Genital Arousal Disorder (PGAD) for at least six months and currently meet the below criteria, you are cordially invited to take part in a research study being conducted by Walden University student, Lisa Squibb as her doctoral dissertation examining PGAD. PGAD Criteria include:

1 - Persistent feelings of arousal extending over days or weeks or months

2 - The arousal is not relieved by or only temporarily relieved by orgasm(s)

3 - The arousal is not associated with sexual thoughts or desire 4 - The arousal may be triggered by nonsexual stimuli or no apparent stimuli

5 - The arousal is bothersome and causes distress

The purpose of this study is to better understand associations of the psychological and relationship contributors to PGAD distress in women. Your participation will contribute greatly to PGAD research and help provide valuable knowledge to clinicians, researchers and people around the globe. If you are interested, please click the following link to begin.

Link to survey/informed consent will be provided here. 
Appendix C: Online Support Group Invitation to Participate

Are you a woman over the age of 17 who has been suffering from Persistent Genital Arousal Disorder (PGAD) for at least six months? If yes, and you also meet the following criteria, you are invited to take part in a research study examining PGAD. PGAD Criteria include:

1 - Persistent feelings of arousal extending over days or weeks or months 2 - The arousal is not relieved by or only temporarily relieved by orgasm(s)

3 - The arousal is not associated with sexual thoughts or desire 4 - The arousal may be triggered by nonsexual stimuli or no apparent stimuli 5 - The arousal is bothersome and causes distress

The study is being conducted as a Ph.D. doctoral dissertation by Walden University student, Lisa Squibb. The purpose of this study is to better understand associations of psychological and relationship contributors to PGAD distress in women. Your participation will contribute greatly to PGAD research and help provide valuable knowledge to clinicians, researchers and people around the globe. If you are interested, please click the following link to begin.

Link to survey/informed consent will be provided here. 
Appendix D: Seven-day Follow-up Email Invitation to Participate

Thank you for your consideration in taking part in the doctoral dissertation research study examining PGAD by Walden University student, Lisa Squibb. If you have already completed the survey, your participation is much appreciated. If you have not had the opportunity, but would still like to participate, this email serves as a reminder that the survey is still open and available for participation.

As a reminder, your participation will contribute greatly to PGAD research and help provide valuable knowledge to clinicians, researchers and people around the globe. If you are interested, please click the following link to begin. The link is provided here again for your convenience.

Link to survey/informed consent will be provided here. 
Appendix E: Fourteen-Day Follow-Up Email Invitation to Participate

Thank you for your consideration in taking part in the doctoral dissertation research study examining PGAD by Walden University student, Lisa Squibb. If you have already completed the survey, your participation is much appreciated. If you have not had the opportunity, but would still like to participate, this email serves as a reminder that the survey is still open and available for participation. The survey will close in two weeks.

As a reminder, your participation will contribute greatly to PGAD research and help provide valuable knowledge to clinicians, researchers and people around the globe. If you are interested, please click the following link to begin. The link is provided here again for your convenience.

Link to survey/informed consent will be provided here. 
Appendix F: Seven-Day Follow-Up Online Support Group Invitation to Participate

Thank you for your consideration in taking part in the doctoral dissertation research study examining PGAD by Walden University student, Lisa Squibb. If you have already completed the survey, your participation is much appreciated. If you have not had the opportunity, but would still like to participate, this notice serves as a reminder that the survey is still open and available for participation.

As a reminder, your participation will contribute greatly to PGAD research and help provide valuable knowledge to clinicians, researchers and people around the globe. If you are interested, please click the following link to begin. The link is provided here again for your convenience.

Link to survey/informed consent will be provided here. 
Appendix G: Fourteen-Day Follow-Up Online Support Group Invitation to Participate

Thank you for your consideration in taking part in the doctoral dissertation research study examining PGAD by Walden University student, Lisa Squibb. If you have already completed the survey, your participation is much appreciated. If you have not had the opportunity, but would still like to participate, this notice serves as a reminder that the survey is still open and available for participation. The survey will close in two weeks.

As a reminder, your participation will contribute greatly to PGAD research and help provide valuable knowledge to clinicians, researchers and people around the globe. If you are interested, please click the following link to begin. The link is provided here again for your convenience.

Link to survey/informed consent will be provided here. 